\title{
La composizione dei Gruppi finiti il cui grado è la quinta potenza di un nu- mero primo.
}

\author{
(Di G. Bagnera, a Palermo.)
}

\section{PREFAZIONE.}

Uaylex ha per il primo posta l'importante questione $\left(^{*}\right)$ di costruire tutti i possibili gruppi di un dato grado $n$.

Non bisogna, nemmeno lontanamente, sperare che il problema, enunciato in termini cosi generali, possa in qualche maniera essere abbordabile: delle ipotesi sono necessarie sopra l'intero $n$ per servire di fondamento ad un ragionamento qualsiasi, e le jpotesi che hanno condotto in questi ultimi anni ai più rimarchevoli risultati si riferiscono al modo con cui l'intero $n$ è composto con i suoi fattori primi.

Il sig. Nerto ha dato (**) tutti i gruppi il cui grado è il prodotto di due soli numeri primi eguali o diseguali; il sig. HöLDer ha, per la prima volta $(* * *)$, dimostrato che tutti i gruppi il cui grado è il prodotto di tre numeri primi, eguali o diseguali, sono metaciclici (risolubili) $\left({ }^{* * * *}\right)$; poi, in un lavoro posteriore $(* * * * *)$, lo stesso Autore ha dato la composizione dei

(*) CAYLEY's Mathemalical papers, Vol. II, 125.

(**) NETTo. Substitutionenhlheorio und ihre Anvendung aur die Algebra, 1882, pag. 133.

(***) HöLDER. Die einfachen Gruppen im ersten und zweiten Hundert der Ordnungszahlen. Mathematische Annalen, Band 40, a. 1892.

(****) Nel presente lavoro mi servo delle denominazioni che, per i gruppi finiti, sono state adottate dal sig. H. Weber nel suo libro: Lehrbuch der Algebra.

(******) HöLDER. Die Gruppen der Ordnungen $p^{3}, p q^{2}, p q r, p^{4}$. Mathematische Annalen, Band 43 , a. 1893. 
gruppi di tale grado e quella di tutti i gruppi, il cui grado è la quarta po.. tenza di un numero primo.

Seguendo lo stesso ordine d'idee, il sig. Frobenrus ha osservato $\left(^{*}\right)$ che tutti i gruppi, il cui grado è il prodotto di quattro fattori primi, sono metaciclici, fatta eccezione per il grado $2^{2} \cdot 3.5=60$ dove si presenta il noto gruppo dell' icosaedro che è un gruppo semplice. Il sig. Frobenius è andato molto più in là : egli ha stabilito $\mathrm{j}$ due sorprendenti risultati generali che ogni gruppo, il cui grado è il prodotto di $\nu$ fattori primi tra cui ve ne siano $\nu-1$ eguali, oppure il prodotto di $\nu$ fattori primi due a due diseguali, è necessariamente metaciclico, e, chi ha interesse di conoscere le ingegnose dimostrazioni di questi due teoremi, potrebbe leggerle nel libro del signor H. WeBER $(* *)$.

Il sig. HöLder, con molta fortuna, è ritornato sulla questione nell'anno $1895\left(^{* * *}\right)$ e, ponendo a fondamento l'ultimo dei due citati teoremi del sig. Frobenus, ha dato la composizione e la classificrzione di tutti i possibili gruppi, il cui grado è il prodotto di quanti si vogliano fattori primi due a due diseguali; inoltre, nello stesso lavoro, ha stabilito il notevolissimo risultato che ogni gruppo di tale grado è necessariamente isomorfo ad un gruppo lineare di permutazioni.

Ma, dare la composizione e la classificazione di tutti i gruppi, il cui grado è il prodotto di quanti si vogliano fattori primi eguali, è un problema di ben altra portata. Su questo riguardo i risultati generali più importanti sono dovuti a Sycow (****). Il sig. HöLDER, nel citato lavoro del 1893, ha formato tutti i gruppi dei gradi $p^{3}$ e $p^{4}$, e, quasi contemporaneamente, è apparso un lavoro del sig. Youva $\left(^{* * * * *}\right)$ il quale, in fondo, si ̀̀ anche limitato a dare la composizione di tutti i gruppi dei gradi $p^{3}$ e $p^{4}$.

Io do, per la prima volta, la composizione di tutti i gruppi di grado $p^{5}$.

(*) Frobesius. Ueber aufösbare Gruppen. Sitzungsherichte der Berliner Akalemie, a. 1893, I, pag. 337.

(**) H. Weber. Lehrbuch der Algetra. Vol. II, jag. 129 e sg.

(***\%) HöLDER. Lie Gruppen nit quadratfreier Ordnungszahl. Göttingor Nachr., a. 1895 .

(****) Sruow. Théorènes sur les groupes de substitutions. Mathematische Annalen, Band $\dot{V}$.

(***) Young. On the determination of Groups whose order is a power of a prime. American Journal of Mathematies, Vol. XV, a. 1893. 
Ł̀ precisamente a partire dal valore $\nu=5$ che il problema della composizione dei gruppi di grado $p^{*}$ mostra la sua vera faccia, giacchè, mentre qualungue sia il numero primo $p$ esistono sempre:

un solo gruppo di grado $p$; due soli gruppi di grado $p^{2}$; cinque soli gruppi di grado $p^{3}$; soltanto quindici gruppi di grado $p^{4}$ tutte le volte che è $p>2$; per i gruppi di grado $p^{5}$ accade invece che il loro numero dipende essenzialmente dal valore del numero primo $p$.

Il caso particolare di $p=2$ è stato tentato dal sig. Levavasseve : egli dice $\left(^{*}\right)$ di aver trovato più di settantacinque gruppi di grado $2^{5}=32$ e di non avere ancora terminata la enumerazione; però, i risultati del sig. Levavasseur sono stati messi in dubbio dal sig. Miluer (**), il quale, sebbene giunga anch' egli a risultati non esatti, si accosta maggiormente al vero; giacchè, come appresso si vedrà, i gruppi di grado 32 sono soltanto in numero di cinquanta.

Nella prima parte di questo lavoro, dopo di avere stabilito nel $\S I$ il principio generale, che serve di fondamento alle successive ricerche, ritrovo anzitutto nel $\S$ II i gruppi di grado $p^{3}$ e $p^{4}$ : ciò è stato necessario per potere, con uniformità di metodo, trattare $\mathrm{i}$ gruppi di grado $p^{\mathrm{s}}$. Le differenze che si potrebbero osservare tra lá tahella che sta alla fine del detto paragrafo ed i risultati dei sig. HöLder e Young non hanno, e si comprende, niente di sostanziale: esso provengono dal fatto che io ho, qualche volta, scelto in modo diverso i tipi ridotti; però, nella maggior parte dẹ casi, per fare il confronto basta cambiare il nome agli elementi generatori.

Nel § III poi mi occupo della composizione dei gruppi, di grado $p^{5}$, limitandomi al caso in cui questi posseggono più d'un divisore Abeliano d'indice $p$.

Nella seconda parte del lavoro, la quale comprende i §§ IV, V, VI e VII, è sviluppata l'analisi che riguarda gli altri casi, ed è data la classificazione completa di tutti i gruppi di grado $p^{5}$.

La classificazione, che ho adottata, a me basta per esser sicuro che nessun gruppo di grado $p^{5}$ mi sia sfuggito, e non mi curo di sapere se questa classificazione sia più o meno conveniente nel caso generale. D'altronde, giacchè in tal caso nulla o poco è conosciuto, una classificazione generale sarebbe cosa perfettamente arbitraria.

(*) Comptes Rendus, a. 1896 , t. 12\%, pag. 182.

(**) Comptes Rendus, a. 1896, t. 122, pag. 370. 
Quantunque io abbia fatto ogni sforzo per dare la massima omogeneità ai procedimenti, debbo confessare che, su questo punto, non ho potuto trionfare giacchè, bo dovuto spesso ricorrere ad artifizi ed a suddivisioni di ripiego anche nella ricerca dei gruppi appartenenti ad una medesima classe; ma, io dubito fortemente che questo fatto sia una necessità inerente alla natura del problema. 


\section{PARTE PRIMA.}

\section{$\S$ I. Generalità.}

1. Sia $P$ un gruppo finito di grado $n$ e $\Gamma$ una classe di $n$ elementi sopra la cui natura non si fa alcuna ipotesi. To suppongo che, in un modo qualunque, si sia stabilita una corrispondenza $S$, univoca e reciproca, tra gli elementi del gruppo $P$ e quelli della classe $\Gamma$ e denoto con $S(e)$ l'elemento di $l$ 'ehe corrisponde all'elemento $e$ di $P$.

Essendo $e^{\prime}$ ed $e^{\prime \prime}$ due arbitrari elementi di $P$, si ponga per definizione:

$$
S\left(e^{\prime}\right) S\left(e^{\prime \prime}\right)=S\left(e^{t} e^{\prime \prime}\right)
$$

allora, rispetto a questa legge di composizione, gli elementi di $\Gamma$ costituiscono un gruppo $G=S(P)$ che si può pensare come il più generale gruppo oloedricamente isomorfo a $P$. Chiamerò brevemente il gruppo $G$ una rapprestntazione di $P$ in 1': cambiando la corrispondenza $S$ cambia, in generale, ]a leggro di composizione degli elementi di $\Gamma$ e si ottiene un'altra rappresentazione di $P^{\prime}$ in $\Gamma$.

Se $T$ è una operazione che permuta gli elementi di I', l'operazione $T S$ stabilisce una nuova corrispondenza tra gli elementi di $P$ e gli elementi di $\Gamma$, e può accadere, in particolare, che le due corrispondenze $S$ ' e $T S$ definiscano la stessa legge di composizione negli elementi di $\mathrm{r}$. Allora, l'operazione $S^{-1} T S$ fia corrispondere ad un elemento di $P$ un elemento di $P$, in modo tale che al prodotto di due elementi corrisponde il prodotto dei due elementi corrispondenti. Esprimerò questo fatto dicendo che l'operazione $S^{-1} T S$ produce una trasformazione d'isomorfismo del gruppo $P$ in sè.

E chiaro che tutte le possibili aperazioni $T$, che corrispondono a trasformazioni d' isomorfismo del gruppo $P$ in sè, costituiscono un gruppo divisore del gruppo simmetrico delle permutazioni di $n$ cifre.

\section{Siano:}

$$
E_{1}, E_{2}, \ldots, E_{\nu}
$$


elementi di $\Gamma$. Se, nella rappresentazione $G=S(P)$, il composto:

$$
E_{1}^{\alpha_{1}} E_{2}^{\alpha_{2}} \ldots E_{2}^{\alpha_{v}}
$$

facendo percorrere ai numeri interi indipendenti $\alpha_{1}, \alpha_{2}, \ldots, \alpha_{4}$ dati sistemi di numeri, fornisce tutti gli elementi di $\Gamma$, e ciascuno una sola volta, dirò che il gruppo $G$ possiede la base (1) a $\nu$ dimensioni.

Denoti :

$$
\Phi\left(E_{1}, E_{2}, \ldots, E_{\nu}\right)=\Psi\left(E_{1}, E_{2}, \ldots, E_{\nu}\right)
$$

una relazione tra gli elementi (1), e si faccia subire agli elementi di $\Gamma$ una qualunque operazione $T$. Allora si passa dalla rappresentazione $S(P)$ alla rappresentazione $T S(P)$ e viene perciò ad essere cambiata la legge di composizione degli elementi di $\Gamma$ : dunque, la relazione (2), che sussiste nella rappresentazione $S(P)$, non si verifica, in generale, nella rappresentazione $T(G)=T S(P)$.

Epperò, una volta fissate alcune relazioni come la (2), jo posso propormi il problema di cercare tutte la possibili operazioni $T$, che conservano le dette relazioni. Queste tail operazioni costituiscono un gruppo, il quale contiene, come divisore, il gruppo formato da tutte le operazioni $T$ che corrispondono a trasformazioni d'isomorfismo del gruppo $P$ in sè.

3. Io mi debbo occupare nel presente lavoro solamente di gruppi $P$. il cui grado è una potenza $p^{\nu}$ di un numero primo $p$ : è quindi conveniente di fissare le idee al caso mio.

Se $G_{\nu}$ è una rappresentazione qualunque di un tale gruppo in $\Gamma$, ̀̀ noto $\left(^{*}\right)$ che è possibile costruire una serie di composizione:

$$
G_{\nu}, G_{\nu-1}, G_{\nu-2}, \ldots, G_{1}
$$

tale che ogni termine della serie sia divisore normale di tutti $\mathrm{i}$ termini che lo precedono. L'ultimo termine $G_{1}$ della serie ì allora un divisore normale di grado $p$ del gruppo $G_{v}$ e quindi, l'elemento generatore $E_{4}$ di $G_{1}$ ha la nota $\left(^{* *}\right)$ proprietà di essere invertibile con tutti gli elementi di $G_{*}$.

(*) V. ad es. una mia Nota: Sopra $i$ divisori normali d'indice primo di un gruppo finito. Rendiconti della R. Accademia dei Lincei. Vol. VII, $2 .^{\circ}$ sem., anı 1898.

(*) Se $E$ è un elemento di $G_{\nu}$ di grado $p^{k}$, dalla relazione $E^{-1} E_{1} E=E_{1}^{\alpha}$ si deduce $\alpha^{p^{k}} \equiv 1(\bmod p)$ e quindi $\alpha \equiv 1(\bmod p)$. 
Gli elementi di $G_{y}$, che godono la detta proprietà, formano evidentemente gruppo e questo gruppo, giacchè contiene $G_{1}$, non può coincidere col gruppo formato dall'elemento identico.

Ciò posto, chiamerò divisore invertibile di $G$, il massimo divisore proprio di $G$, tale che ogni suo elemento è invertibile con qualunque elemento del gruppo principale $G_{y}$, e supporrò, cosa sempre possibile, che questo divisore faccia parte della serie di composizione (3).

Bisogna osservare che il divisore invertibile $H$ di un gruppo $G_{v}$ è perfettamente determinato, tranne il caso in cui $G_{\nu}$ è un gruppo Abeliano: allora si può scegliere come gruppo $H$ un divisore qualunque d'indice $p$ di $G_{\nu}$.

Se $p^{h}$ è il grado di $H$, il numero:

$$
\mu=\nu-h-1
$$

¿̀ un numero invariantivo del gruppo $G_{y}$ e questo numero è, per quel che segue, della massima importanza.

Un gruppo $G_{\nu}$, che ha il numero invariantivo $\mu$, sarà denotato con $G_{\nu}{ }^{\mu}$; così, ad es., i gruppi $G_{*}{ }^{0}$ sono gruppi Abeliani.

4. Nella Nota citata precedentemente, supponendo che un gruppo finito $G$ possegga divisori normali d'indice primo $p$, ho chiamato indipendenti $\lambda$ di tali divisori, quando la loro intersezione $K$ ha in $G$ l'indice $p^{2}$, ed ho fatto vedere che, se $\lambda$ è il numero totale dei divisori normali indipendenti d'indice $p$ contenuti in $G$, questo possiede $\frac{p^{2}-1}{p-1}$, e non più, divisori normali d'indice $p$.

Inoltre ho dimostrato che il gruppo complementare $G \mid K$ è Abeliano ed ha tutti $i$ suoi elementi di grado $p$; dunque, due arbitrari elementi del gruppo $G$ sono invertibili rispetto a mod. $K$ e le potenze $p^{\text {me }}$ di tutti gli elementi di detto gruppo appartengono a $K$.

In un gruppo $G_{,}$, di grado $p^{\nu}$, ogni divisore d'indice $p$ è normale, quindi si ha $\lambda>1$, ed io dico che si ha $\lambda=1$ solamente quando il gruppo $G_{\nu}$ è ciclico.

Siccome la proposizione si verifica per i gruppi di grado $p^{2}$, basta provare che, ammettendola vera per $\mathrm{i}$ gruppi di grado $p^{\nu-1}$, sussiste ancora per i gruppi di grado $p^{*}$. Si chiami $G_{1}$ un divisore normale di grado $p$ del gruppo $G_{\nu}$ : se questo gruppo ha un solo divisore d'indice $p$, anche il gruppo $G_{v} ! G_{1}$ complementare di $G_{1}$, ha un solo divisore d'indice $p$, e giacchè detto gruppo complementare è di grado $p^{\nu-1}$, esso è ciclico per ipotesi. Sia dunque 
$E$ un elemento di $G$, di grado $p^{\gamma-1}$ rispetto a mod. $G_{1}$ ed $E_{1}$ un elemento generatore di $G_{i}$. Se fosse $E p^{\prime-1}=1$, il gruppo $G_{*}$, contrariamente all'ipotesi, ammetterebbe il divisore d'indice $p$ generato dall' elemento $E$ ed il divisore d'indice $p$ generato dai due elementi $E p$ ed $E_{1}$. Dunque, l'elemento $E$

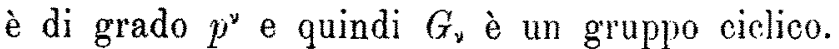

Cio posto, di ogni gruppo $G y^{\mu}$ jo do anche il numero $\lambda$ dei divisori indjpendenti d'indice $p$ che il detto gruppo possiede e convengo di denotare $G_{v}{ }^{\prime \prime}$ con $G_{v}{ }^{\mu \lambda}$ quando voglio mettere in evidenza il numero invariantivo $\lambda$.

È utile osservare che, se è $\mu>0$, deve essere $2 \leq \lambda<\nu$ : ciò risulta dalle cose ultimamente dette.

Inoltre si noti che, nell'ipotesi di $\mu>0$, il gruppo $G_{y} H$ non è ciclico, e quindi esistono almeno due distinti divisori d'indice $p$ di $G$, che contengono i) gruppo $H$.

5. Ritornando alla serie (3), che io chiamo una serie canonica di composizione di $G_{\eta}$, sia $E_{i}\left(i=1,2, \ldots\right.$, , ) un elemento di $G_{i}$ fuori di $G_{i-1}^{i}$ e $G_{0}$ il gruppo formato dall elemento identico: siano poi $E^{\prime}{ }_{i}, F_{i}^{\prime \prime}{ }_{i}, \ldots$ elementi qualunque di $G_{i}$.

Ciò posto, giacchè il gruppo $G_{i} G_{i-1}$ è un divisore normale di grado $p$ del gruppo $G_{y}, G_{i-1}$, risulta che l'elemento $E_{i}$ è invertibile con ogni e]emento del gruppo $G_{v}$ rispetto a mod. $G_{i-1}$; dunque, in particolare, supponendo $j>i$, si ha:

$$
E_{j}^{-1} E_{i}^{\prime} E_{j}^{\prime}=E_{i} E_{i 1}^{\prime}, \quad E_{i}^{\prime \prime}=E^{\prime \prime}{ }_{i-1}
$$

dove gli elementi $E_{i-1}$ ed $E_{i-1}^{\prime \prime}$ appartengono, per quello che si è detto nel $\mathrm{n}^{\circ}$ precedente, al gruppo $K$ intersezione dei divisori d'indice $p$ del gruppo $G$..

Data la serie di composizione (3) e, relativamente ad essa, definendo gli elementi :

$$
E_{\nu}, E_{\nu-1}, \ldots, E_{1}
$$

nell'anzidetto modo, il composto :

$$
E_{* \prime \prime}^{\prime \prime} E_{,-1}^{\prime \prime \prime} \ldots E_{1,}^{\prime \prime}
$$

fornisce tutti gli elementi del gruppo $G_{\nu}$, e ciascuno una sola volta, attribuendo agli interi $\alpha_{\nu}, \alpha_{\nu-1}, \ldots, \alpha_{1}$ valori scelti ad arbitrio nel sistema di numeri $0,1,2, \ldots, p-1$. Dunque, gli elementi $E_{\nu}, E_{\nu-1}, \ldots, E_{1}$ costituiscono una base a $\nu$ dimensioni del gruppo $G$, e tra $\mathrm{i}$ detti elementi sussistono le relazioni (4). Una tale base la chiamerò una base canonica $\left(^{*}\right)$ di $G_{y}$.

(*) L'esistenza di una base canonica per un trupuo di grado $y^{\nu}$ è stata, per la prirna volta, dimostrata da SYLow, l. c. 
Quando si conosce una base canonica di $G$, è perfettamente individuata una serie canonica di composizione del gruppo $G_{\nu} ;$ ma, in corrispondenza ad ognuna di dette serie, si possono definire più basi canoniche.

Sia :

$$
\mathbf{E}_{v}, \mathbf{F}_{v-1}, \ldots, \mathbf{E}_{1}
$$

una base canonica di $G_{\nu}$ diversa dalla precedente. L'operazione che porta ogni

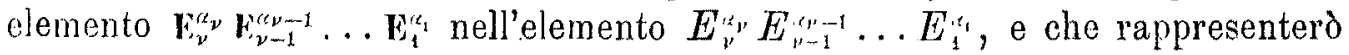
con la notazione:

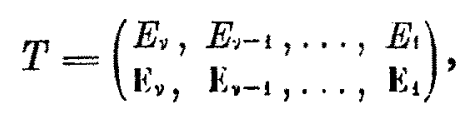

lascia inalterata la forma di tutte le relazioni (4), ma cambia, in generale, gli elementi $E_{i}^{\prime}$ ed $E^{\prime \prime}{ }_{i}$ che ivi figurano. Si può allora scegliere l'operazione $T$ in modo che la rappresentazione $T\left(G_{\nu}\right)$ sia, da un dato punto di vista, più ('onveniente della rappresentazione $G_{*}$.

Ma ciò che è ancora più importante è il fatto che, date due rappresentarioni dello stesso gruppo $P$, in $\Gamma$ e scegliendo in ciascuna di esse una base canonica, si può passare dalla serie di relazioni (4) relativa alla prima rapfresentazione alla serie di relazioni (4) relativa alla seconda rappresentazione modiante una operazione $T$ appartenente al gruppo delle operazioni che portano una base canonica in una base canonica.

\section{$\S$ II. I Gruppi di grado $p^{3}$ e $p^{4}$.}

6. Esistono soltanto tre gruppi $G_{3}{ }^{0}$, i quali sono:

un gruppo $G_{3}^{0.4}$, che è il gruppo ciclico;

un gruppo $G_{3}{ }^{0,2}$, che ha un elemento di grado $p^{2}$ ed un elemento di grado $p$ il quale non è una potenza del primo; un gruppo $G_{3}{ }^{n .3}$, che ba tutti $i$ suoi elementi di grado $p$.

Mettendo in evidenza, per ciascuno di questi tre gruppi, gl'invarianti del sigr. Frobenius (*), scriverò :

$$
\left.\left.G_{3}^{0,1} \mid\left[p^{3}\right]\right\}, \quad G_{3}^{0,2}\left\{\left[p^{2}\right][p]\right\}, \quad G_{3}^{0,3} \mid[p][p][p]\right\} .
$$

(*) Frobexius u. Strokelberaer. Crelle's Journal, Vol. LXXXVI, pp. 224-236. 
Se :

7. I gruppi $G_{3}$ che non sono Abeliani sono gruppi $G_{3}{ }^{1{ }^{2}}$.

$$
E_{3}, E_{2}, E_{1}
$$

è una base canonica di un tale gruppo, con le notazioni del paragrafo precedente si ha $G_{1}=H=K$, e quindi è :

$$
\begin{gathered}
E_{3}^{-1} E_{2} E_{3}^{i}=E_{2}^{i} E_{1}^{u}, \quad E_{3}^{-1} E_{1} E_{3}=E_{1}, \quad E_{2}^{-1} E_{1} E_{2}=E_{1} ; \\
E_{3}^{p}=E_{1}^{\prime}, \quad E_{2}^{p}=E_{1}^{i}, \quad E_{1}^{p}=1 ;
\end{gathered}
$$

dove il numero $\alpha$ è primo con $p$. Allora, dati i due elementi $E_{3}, E_{2}$, si può definire l'elemento $E_{1}$ mediante la relazione:

$$
E_{3}^{-1} E_{2} E_{3}=E_{2} E_{1}
$$

e percio, nelle relazioni $(1)$, ritengo $\alpha \equiv 1(\bmod p)$.

Io ho, in questo modo, dimostrato che, se esiste un gruppo $P_{3}$ rappresentato in $\mathrm{T}$ da un $G_{3}{ }^{1,2}$, gli elementi $E_{3}, E_{2}, E_{1}$ di una base canonica del gruppo rappresentativo debbono soddisfare le (1). Bisogna pertanto provare che un tale gruppo $P_{3}$ effettivamente esiste.

Si considerino come elementi tutti i simboli :

$$
e_{3}^{x} e_{2}^{y} e_{1}^{z}
$$

che si ottengono attribuendo agli esponenti $x, y, z$ arbitrari valori interi, e si chiamino eguali due tali simboli quando si deducono l'uno dall'altro facendo variare ordinatamente $\mathrm{i}$ detti esponenti di $p k_{3}, p k_{2}, h_{1}$, purchè gl'interi $h_{1}$, $k_{2}, k_{3}$ soddisfino alla congruenza :

$$
h_{1}+\beta k_{2}+\gamma k_{3} \equiv 0 \quad(\bmod p) \text {. }
$$

Si ottengono cosi soltanto $p^{3}$ elementi distinti.

Cosi posto, se si assume come definizione del prodotto di due elementi la relazione:

$$
\left(e_{3}^{x^{\prime}} e_{2}^{y^{\prime}} e_{1}^{z}\right)\left(e_{3}^{x^{\prime \prime}} e_{2}^{y^{\prime \prime}} e_{1^{z^{\prime \prime}}}\right)=e_{3}^{x^{\prime}+x^{\prime \prime}} e_{2}^{y^{\prime}+y^{\prime \prime}} e_{1}^{z^{\prime}+z^{\prime \prime}+y^{\prime \prime \prime}},
$$

riesce facile verificare che, essendo $A, B, C$ elementi (3), sono soddisfatte le condizioni :

$1 .^{\mathrm{a})}$ se è $A=B$, ̀̀ $A C=B C$ e $C A=C B$,

$2 .^{\text {a) }}$ se è $A C=B C$ oppure $C A=C B$, ̀̀ $A=B$,

$\left.3 .{ }^{\mathrm{a}}\right)$ in ogni caso, ̀̀ $(A B) C=A(B C)$. 
I simboli (3), rispetto alla legge di composizione (4), costituiscono dunque un gruppo $P_{3}$, di cui l'elemento unità è il simbolo $e_{3}^{0} e_{2}^{0} e_{1}^{0}$, e se si rappresenta detto gruppo in $\Gamma$ facendo corrispondere agli elementi $e_{3}^{1} e_{2}{ }^{0} e_{1}{ }^{0}, e_{3}{ }^{0} e_{2}{ }^{1} e_{1}{ }^{0}$, $e_{3}{ }^{0} e_{2}{ }^{0} e_{1}{ }^{1}$ ordinatamente gli elementi $E_{3}, E_{2}, E_{1}$, questi costituiscono una base canonica di un gruppo $G_{3}{ }^{1,2}$ e soddisfano alle relazioni (1), le quali perciò, nel senso del sig. Dxck $\left(^{*}\right)$, definiscono sempre un gruppo $G_{3}^{1,2}$ qualunque siano gl'interi $\beta$ e $\gamma$.

8. Ma, giacchè non sono da considerarsi come gruppi distinti le diverse rappresentazioni di uno stesso gruppo, bisogna vedere come variano $\beta$ e $\gamma$ relativamente al gruppo delle operazioni :

$$
T=\left(\begin{array}{lll}
E_{3}, & E_{2}, & E_{1} \\
\mathbf{E}_{3}, & \mathbf{E}_{2}, & \mathbf{E}_{1}
\end{array}\right)
$$

che lasciano inalterata la (2).

Ponendo:

$$
\mathbf{E}_{3} \equiv E_{3}{ }^{u} F_{2}{ }^{v}, \quad \mathbf{E}_{2} \equiv E_{3}{ }^{r} E_{2}{ }^{s}, \quad\left(\bmod G_{1}\right)
$$

affinchè la relazione (2) si conservi, bisogna prendere $\mathbf{E}_{1}=E_{1}{ }^{A}$; quindi, per non contraddire alla definizione dell'elemento $\mathbb{F}_{4}$, deve essere il determinante $\Delta=u s-v r$ primo con $p$.

D'altra parte, io osservo che le operazioni $T$ appartenenti alla classe definita dalle relazioni:

$$
\mathbf{E}_{3} \equiv E_{3}, \quad \mathbf{E}_{2} \equiv E_{2}, \quad\left(\bmod G_{1}\right),
$$

non alterano $\beta$ e $\gamma$ e costituiscono un divisore normale del gruppo delle operazioni $T$ definito precedentemente; dunque, rispetto alla questione di cui mi sto occupando, posso ritenere eguale all'operazione identica ogni operazione di detta classe. In questa ipotesi, le (5) definiscono un'unica operazione che io rappresento con la notazione:

$$
\left|\begin{array}{ll}
u & v \\
r & s
\end{array}\right|
$$

Ora, si osservi che la relazione (2) dà subito:

$$
\left(E_{3} E_{2}\right)^{n}=E_{3}{ }^{n} E_{2}{ }^{n} E_{1}^{\left(\begin{array}{l}
n \\
2
\end{array}\right)}
$$

(*) W. DYck. Gruppentheoretische Sludien. Mathematische Annalen, Band XX, a. 1882. 
e quindi, se ̀̀ $p>2$, si ha :

$$
\mathbf{E}_{3}^{p}=E_{3}^{u p} E_{2}^{v p}=E_{1}^{r u+\beta v}, \quad \mathbf{E}_{2}^{p}=E_{3}^{r p} E_{2}^{s p}=H_{1}^{r+\beta s}
$$

Dunque, se $\beta_{1}$ e $y_{1}$ sono i nuovi valori di $\beta$ e $\gamma$ dopo avere eseguita la detta operazione, si trova:

$$
\Delta \gamma_{1} \equiv \gamma u+\beta v, \quad \Delta \beta_{1} \equiv \gamma v+\beta s, \quad(\bmod p) .
$$

Allora, se $\beta$ e $\gamma$ sono entrambi nulli $(\bmod p)$, lo stesso accade per $\beta_{1}$ e $\gamma_{1}$ ed il gruppo $G_{3}{ }^{1,2}$ ha tutti i suoi elementi di grado $p$; in caso contrario una almeno delle due operazioni :

$$
\left|\begin{array}{rrr}
\beta & -i \\
0 & i
\end{array}, \quad \vdots \begin{array}{rr}
i & -i \\
1 & 0
\end{array}\right|,
$$

ha il determinante primo con $p$, e questa porta $\beta$ e $\%$ ordinatamente nei resti 1 e 0 presi rispetto al modulo $p$.

In conclusione esistono, se è $p>2$, due soli gruppi $G_{3}^{1,2}$ in corrispondenza alle due serie di formole:

$$
\left.\begin{array}{ll}
E_{3}^{p}=1, & E_{:}^{p}=1, \quad E_{1}^{p}=1 ; \\
E_{3}^{p}=1, \quad E_{2}^{p}=E_{1}, \quad E_{1}^{p}=1 ;
\end{array}\right\}
$$

e, tralasciando di scrivere le relazioni che esprimono che l'elemento $E_{1}$ appartiene al divisore invertibile $H$, questi due gruppi sono perfettamente determinati dalle relazioni (7) insieme alla (2).

9. Il caso di $p=2$ esige una breve analisi particolare. In questa ipotesi, tutte le possibili operazioni :

$$
\begin{array}{ll:}
u & v \\
r & s
\end{array} ;
$$

formano un gruppo di grado sei che può essere generato dalle due operazioni :

$$
\begin{array}{ll}
1 & 1 \\
0 & 1
\end{array}|,| \begin{array}{ll}
0 & 1 \\
1 & 1
\end{array} \mid
$$

di secondo e terzo ordine rispettivamente.

Osservando che la (6) dà :

$$
\left(E_{3} E_{2}^{2}\right)^{2}=E_{3}^{2} E_{2}^{2} E_{1}
$$

si vede che l'ipotesi di $E_{2}{ }^{2}=E_{1}$ ed $E_{3}{ }^{2}=E_{1}$ non è cambiata dalle dette due 
operażioni; mentre qualunque altro caso si può ridurre a quello in cui è $E_{2}^{2}=1$ ed $E_{3}^{2}=1$.

Dunque, anche quando è $p=2$, esistono due soli gruppi $G_{3}^{1,2}$ di grado $2^{3}=8$ definiti dalle due serie di formole :

$$
\begin{array}{lll}
E_{3}^{2}=1, & E_{2}^{2}=1, & E_{1}^{2}=1 ; \\
E_{3}^{2}=E_{1}, & E_{2}^{2}=E_{1}, & E_{1}^{2}=1,
\end{array}
$$

e dalla relazione (2).

10. Passo ora ad occuparmi dei gruppi di grado $p^{4}$ cominciando a scrivere senz'altro i gruppi di tale grado che sono Abeliani.

Esistono soltanto cinque gruppi $G_{4}{ }^{\circ}$ i quali sono:

un gruppo $G_{4}^{0,1}$, due gruppi $G_{4}^{0,2}$, un gruppo $G_{4}^{0,3}$ ed un gruppo $G_{4}^{0,4}$.

Mettendo in evidenza i rispettivi invarianti, rappresenterò i detti gruppi nel seguente modo:

$$
\begin{gathered}
G_{4}^{0,4}\left\{\left[p^{4}\right]\right\}, \quad G_{4}^{0,2}\left\{\left[p^{3}\right][p]\right\}, \quad G_{4}^{0,2}\left\{\left[p^{2}\right]\left[p^{2}\right]\right\}, \\
G_{4}^{0,3}\left\{\left[p^{2}\right][p][p]\right\}, \quad G_{4}^{0,4}\{[p][p][p][p]\} .
\end{gathered}
$$

Il gruppo $G_{4}^{0,1}$ è il gruppo ciclico di grado $p^{\mathbf{4}}$; il primo dei due gruppi $G_{4}{ }^{0,2}$ ha $p$ divisori $G_{3}{ }^{0,1}$ ed un solo divisore $G_{3}^{0,2}$, inentre il secondo ha $p+1$ divisori $G_{3}{ }^{0,2}$; il gruppo $G_{1}{ }^{0,3}$ ha un solo divisore $G_{3}{ }^{0,3}$ e $p^{2}+p$ divisori $G_{3}{ }^{0,2}$; finalmente, tutti $\mathrm{i} \frac{p^{4}-1}{p-1}$ divisori d'indice $p$ del gruppo $G_{4}^{0,4}$ sono gruppi $G_{3}^{0,3}$.

11. I gruppi $G_{4}$ che non sono Abeliani sono gruppi $G_{4}{ }^{1}$ oppure gruppi $G_{4}{ }^{2}$ : io comincio ad occuparmi dei primi.

Sia:

$$
G_{1}, G_{3}, G_{2}, G_{1},
$$

una serie canonica di composizione di un gruppo $G_{4}{ }^{1}$ scelta in modo che si abbia $G_{2}=H$.

Giacchè esistono (n. $\left.{ }^{\circ} 4\right)$ due divisori d'indice $p$ di $G_{4}{ }^{1}$ che contengono $H$, il gruppo $K$, o coincide con $H$ oppure è un divisore proprio di $H$, secondo che $G_{1}{ }^{1}$ è un $G_{1}^{1,2}$ od un $G_{4}^{{ }^{1}, 3}$.

Cid posto, se :

$$
E_{1}, \quad E_{3}, \quad E_{2}, \quad E_{1},
$$

denota una base canonica relativa alla precedente serie di composizione, si ha:

$$
E_{4}^{-1} E_{3} E_{4}=E_{3} E_{2}^{\prime}, E_{4}^{\prime \prime}=E_{2}^{\prime \prime}, E_{3}^{p}=E_{2}^{\prime \prime \prime}, E_{2}^{p}=E_{1}^{\prime}, E_{1}^{p}=1 \text {. }
$$


Trattandosi di un gruppo $G_{4}{ }^{4}$, l'elemento $E_{3}$ non è invertibile con $E_{4}$, mentre il contrario accade per l'elemento $E_{3}^{p}$ che appartiene ad $H$; quindi, dalla prima delle (8), risulta che $E_{\mathrm{g}}^{\prime}$ è un elemento di grado $p$ ed io posso ritenere $E_{2}^{\prime}=E_{1}$. Allora, la prima delle formole (8) si può sostituire con :

$$
E_{4}^{-1} E_{3} E_{4}=E_{3} E_{1} \text {. }
$$

Se il gruppo $H=G_{2}$ è ciclico, $E_{3}^{\prime \prime}$ ed $E_{2}{ }^{\prime \prime \prime}$ sono potenze di $E_{2}$; quindi, scegliendo $E_{2}$ in modo che sia $E_{2}^{p}=E_{1}$, le ultime quattro delle relazioni (8) si possono, in questo caso, sostituire con :

$$
E_{4}^{p}=E_{2}^{a}, \quad E_{3}^{p}=E_{2}^{\rho}, \quad E_{2}^{p}=E_{1}, \quad E_{1}^{p}=1 \text {. }
$$

Dopo ciò, si può subito dimostrare che le formole $(9)$ e $(10)$, insieme a quelle che esprimono che l'elemento $E_{z}$ appartiene ad $H$, definiscono, qualunque siano gl'interi o e $\beta$, un gruppo $G_{4}{ }^{1}$.

Si considerino $\mathrm{i}$ simboli che si ottengono da:

$$
e_{4}^{x} e_{3}^{y} e_{2}^{z} e_{1}^{t} \text {, }
$$

attribuendo ad $x, y, z, t$ arbitrari valori interi, e si chiamino eguali due tali simboli quando si possono ottenere l'uno dall'altro facendo variare i detti interi ordinatamente di $p k_{4}, p k_{3}, h_{2}, h_{1}$, purchè sia:

$$
\alpha k_{4}+\beta k_{3}+h_{2}+p h_{1} \equiv 0, \quad\left(\bmod p^{2}\right) \text {. }
$$

Dietro questa convenzione si ottengono solamente $p^{4}$ simboli distinti.

Ora, ponendo per definizione:

$$
\left(e_{4}^{x^{\prime}} e_{3}^{y^{\prime}} e_{2}^{z^{\prime}} e_{1}^{t^{\prime}}\right)\left(e_{4}^{x^{\prime \prime}} e_{3} y^{\prime \prime} e_{2}^{z^{\prime \prime}} e_{1}^{t^{\prime \prime}}\right)=e_{4}^{x^{\prime}+x^{\prime \prime}} e_{3}^{y^{\prime}+y^{\prime \prime}} e_{2}^{z^{\prime}+z^{\prime \prime}} e_{1}^{t+t^{\prime}+y^{\prime} x^{\prime \prime}},
$$

riesce facile verificare che, rispetto a questa legge di composizione i simboli $e_{4}^{x} e_{3}^{y} e_{2}^{z} e_{1}^{t}$ costituiscono un gruppo. Se si rappresenta questo gruppo in $\Gamma$ facendo corrispondere ai simboli:

$$
e_{4}{ }_{1}^{1} e_{3}{ }^{0} e_{2}{ }^{0} e_{1}{ }^{0}, \quad e_{4}{ }^{0} e_{3}{ }^{1} e_{2}{ }^{0} e_{4}{ }^{0}, \quad e_{4}{ }^{0} e_{3}{ }^{0} e_{2}{ }^{1} e_{4}{ }^{0}, \quad e_{4}{ }^{0} e_{3}{ }^{0} e_{2}{ }^{0} e_{4}{ }^{1},
$$

ordinatamente gli elementi $E_{4}, E_{3}, E_{2}, E_{1}$, questi costituiscono una base canonica di un gruppo $G_{*}$ e verificano le relazioni $(9)$ e (10).

Si osservi che l'operazione:

$$
\left(\begin{array}{cccc}
E_{4} & E_{3} & E_{2} & E_{1} \\
E_{4} E_{2}^{\lambda} & \dot{E}_{3} E_{3}^{\prime \prime} & E_{2} & E_{4}
\end{array}\right)
$$

cambia $\alpha$ e $\beta$ rispettivamente in $\alpha+\lambda p$ e $\beta+\mu p ;$ quindi, se $\alpha$ e $\beta$ sono multipli di $p$, si può supporre $\alpha=\beta=0$. 
Se uno dei due numeri $\alpha, \beta$ è primo con $p$, io suppongo che sia $\beta$ primo con $p$ giacchè, in caso contrario, scambio $\alpha$ in $\beta$ mediante l'operazione:

$$
\left(\begin{array}{llll}
E_{4} & E_{3} & E_{2} & E_{1} \\
E_{3}^{-1} & E_{4}^{-1} & E_{2}^{-1} & E_{1}^{-1}
\end{array}\right)
$$

poi, eseguendo la trasformazione:

$$
\left(\begin{array}{llll}
E_{1} & E_{3} & E_{2} & E_{1} \\
E_{4}^{\beta} & E_{3} & E_{2}^{\beta} & E_{1}^{\beta}
\end{array}\right)
$$

mi riduco al caso di $\beta=1$. Ciò posto, cambiando l'elemento $E_{4}$ in $E_{4} E_{3}^{-\alpha}$. si vede facilmente che si può sempre ritenere $\alpha=0$.

Esistono perciò due soli gruppi $G_{1}^{1}$ che hanno il divisore invertibile ciclico e questi due gruppi sono definiti dalla (9) e dalle seguenti modificazioni delle $(10)$ :

$$
\begin{array}{ll}
E_{1}^{p}=1, \quad E_{3}^{p}=1, \quad E_{2}^{p}=E_{1}, \quad E_{1}^{p}=1 \\
E_{4}^{p}=1, \quad E_{3}^{p}=E_{2}, \quad E_{2}^{p}=E_{1}, \quad E_{1}^{p}=1
\end{array}
$$

Il primo di questi gruppi è un $G_{4}^{1,3}$ e fra tutti i suoi divisori d'indice $p$ ve ne sono $p^{2}$ non Abeliani; il secondo gruppo è un $G_{4}^{1,2}$ e tutti i suoi $p+1$ divisori d'indice $p$ sono Abeliani.

12. Si supponga ora che il gruppo $H=G_{2}$ non sia ciclico. Allora le ultime quattro delle relazioni (8) si scrivono:

$$
E_{4}^{p}=E_{2}^{\alpha} E_{1}^{\beta}, \quad E_{3}^{p}=E_{2}^{y} E_{1}^{\delta}, \quad E_{2}^{p}=1, \quad E_{1}^{p}=1 .
$$

Per dimostrare che le formole (9) e (11) definiscono, in ogni caso, un gruppo $G_{4}{ }^{3}$, basta ripetere il ragionamento del $\mathrm{n} .^{\circ}$ precedente, convenendo però, per andare d'accordo con le (11), di ritenere eguale al simbolo:

$$
e_{1}^{x} e_{3}^{y} e_{2}^{z} e_{1}^{t},
$$

ogni altro che da quello si ottiene facendo variare gli esponenti ordinatamente di $p k_{1}, p k_{3}, h_{2}, h_{1}$ in modo che siano soddisfatte le congruenze:

$$
\left.\begin{array}{l}
\alpha k_{4}+\gamma k_{3}+h_{2} \equiv 0 \\
\beta k_{4}+\delta k_{3}+h_{1} \equiv 0
\end{array}\right\}(\bmod p) .
$$

Se $i l$ gruppo $G_{1}{ }^{1}$ è un $G_{1}^{1,2}$, almeno uno degl' interi $\alpha, \gamma$ deve essere primo con $p$ ed io suppongo che sia $\gamma$ primo con $p$. Allora si può definire l'ele- 
mento $E_{2}$ mediante la relazione $E_{3}^{p}=E_{2}$; poi, chiamando $E_{1}$ l'elemento $E_{4} E_{3}{ }^{-a}$ mi riduco al caso di $\alpha=0$. Ciò posto, se non è $\beta$ un multiplo di $p$, la trasformazione:

$$
\left(\begin{array}{llll}
E_{1} & E_{3} & E_{2} & E_{1} \\
E_{1} & E_{3}^{\beta} & E_{2}^{\beta} & E_{1}^{\beta}
\end{array}\right)
$$

porta $\beta$ in 1 .

Esistono perciò due gruppi $G_{4}^{1,2}$ tali ohe il divisore invertibile abbia tutti i suoi elementi di grado $p$ e questi due gruppi corrispondono alle seguenti modificazioni delle formole (11):

$$
\begin{array}{ll}
E_{4}^{p}=1, \quad E_{3}^{p}=E_{2}, \quad E_{2}^{p}=1, & E_{1}^{p}=1 \\
E_{4}^{p}=E_{1}, & E_{3}^{p}=E_{2}, \quad E_{2}^{p}=1, \quad E_{1}^{p}=1
\end{array}
$$

Se il gruppo $G_{4}{ }^{1}$ è un gruppo $G_{4}{ }^{1.3}$, nelle (11) bisogna supporre $\alpha=\gamma=0$, ed allora è necessario distinguere il caso di $p>2$ dal caso di $p=2$.

Nel primo caso, se è $\beta=\delta^{\natural}=0$, il gruppo $G_{4}{ }^{1,3}$ ha tutti i suoi elementi di grado $p$, e ciò risulta dalla formola:

$$
\left(E_{4} E_{3}\right)^{n}=E_{4}^{n} E_{3}^{n} E_{1}^{\left(\begin{array}{l}
n \\
2
\end{array}\right)}
$$

se invece uno dei due numeri $\beta$, $\partial$ è primo con $p$, io posso supporre che sia $\partial$ primo con $p$. Allora, facendo la trasformazione:

$$
\left(\begin{array}{llll}
E_{1} & E_{3} & E_{2} & E_{1}^{\prime} \\
E_{4}^{\delta} & E_{3} & E_{2} & E_{1}^{\dagger}
\end{array}\right)
$$

mi riduco al caso di $\delta=1$; poi, chiamando $E_{4}$ l'elemento $E_{4} E_{3}-\beta$ porto $\beta$ in 0 .

Esistono quindi, nel presente caso, due soli gruppi $G_{4}^{1,3}$ i quali sono definiti dalle formole:

$$
\begin{aligned}
& E_{1}^{p}=1, \quad E_{3}^{p}=1, \quad E_{2}^{p}=1, \quad E_{1}^{p}=1 \\
& E_{4}^{p}=1, \quad E_{3}^{p}=E_{1}, \quad E_{2}^{p}=1, \quad E_{1}^{p}=1
\end{aligned}
$$

e dalla formola (9).

Nell' ipotesi di $p=2$, tenendo presente la (12), si vede subito che il caso $E_{4}^{2}=E_{1}, E_{3}^{2}=E_{1}$ è irreduttibile, mentre qualunque altro caso si riduce a quello in cui ̀̀ $E_{4}^{?}=1, E_{3}^{?}=1$. 
Dunque, anche quando è $p=2$, esistono due soli gruppi $G_{1}^{1,3}$ tali che il divisore invertibile non è ciclico e questi due gruppi corrispondono alle seguenti modificazioni delle formole (11):

$\mid \begin{array}{ccc}E_{1}^{2}=1, & E_{3}^{2}=1, \quad E_{3}^{2}=1, & E_{1}^{2}=1 \\ E_{4}^{2}=E_{1}, & E_{3}^{2}=E_{1}, \quad E_{2}^{2}=1, \quad E_{1}^{2}=1\end{array}$

13. Ritornando alla distinzione fatta al principio del $n .^{0} 11$, io mi voglio ora occupare dei gruppi $G_{4}$ che sono $G_{4}{ }^{2}$ e comincio col dimostrare che un tale gruppo possiede sempre un divisore Abeliano d'indice $p$ ed uno solo (*).

Sia :

$$
G_{1}^{2}, \quad G_{3}, \quad G_{2}, \quad G_{1}
$$

una serie canonica di composizione di un gruppo $G_{4}^{2}$ e si considerino le classi distinte di elementi:

$$
G_{2}^{\prime}, \quad E^{\prime} G_{2}, \quad E^{\prime \prime} G_{z}, \ldots
$$

contenute in $G_{4}{ }^{2}$. Ognuno degli elementi $E^{\prime}, E^{\prime \prime}, \ldots$ deve trasformare un elemento $E_{2}$ di $G_{2}$ nel prodotto di $E_{2}$ per una potenza di $E_{1}$; ora, giacchè gli elementi $E^{\prime}, E^{\prime \prime}, \ldots$ sono in numero di $p^{2}-1$, necessariamente esistono due tali elementi, per es. $E^{\prime}, E^{\prime \prime}$, che trasformano $E_{2}$ nel prodotto di $E_{2}$ per una stessa potenza di $E_{1}$; quindi l'elemento $E^{\prime-1} E^{\prime \prime}$, che non sta in $G_{2}$, è invertibile con $E_{2}$. Se chiamo $E_{3}$ il detto elemento, i tre elementi $E_{1}, E_{2}, E_{3}$, permutabili due a due, generano un gruppo Abeliano il cui grado non è minore, e non pud essere maggiore di $p^{3}$.

Se il gruppo $G_{1}{ }^{2}$ avesse due divisori Abeliani di grado $p^{3}$, la loro intersezione, che è di grado $p^{2}$, dovrebbe essere contenuta nel gruppo $H$ il quale è attualmente di grado $p$ : dunque la mia tesi è dimostrata.

Ciò posto, io dico che ogni gruppo $G_{4}{ }^{2}$ è un gruppo $G_{4}^{2,2}$. Infatti, se fosse possibile un gruppo $G_{1}^{2 ; 3}$, per un tal gruppo sarebbe $K=H=G_{1}$ e quindi sarebbe:

$$
E_{4}^{-1} E_{3} E_{4}=E_{3} E_{1}^{\alpha}, \quad E_{\mathrm{d}}^{-1} E_{8} E_{4}=E_{2} E_{1}^{\beta}
$$

(*) Questo risultato è stato comunicato per lettera dal sig. HöLder al sig. MiLLER. Ofr. Mrler. Sur les groupes de substitutions. Comptes Rendus, t. 122, a. 1896, pag. 371. 
con $\beta$ primo con $p$. Allora, scegliendo l'intero $m$ in modo che sia $\beta m+\alpha \equiv 0$ (mod $p$ ), l'elemento $E_{3} E_{2}{ }^{m}$ risulta permutabile con $E_{4}$ e quindi $G_{3}$ non sarebbe il solo divisore Abeliano d'indice $p$ contenuto in $G_{4}^{2,3}$.

Le considerazioni che precedono mostrano che è possibile determinare una base canonica:

$$
E_{4}, E_{3}, E_{2}, E_{1}
$$

di un gruppo $G_{4}^{2}$ in modo tale che sia:

$$
E_{4}^{-1} E_{3} E_{4}^{\prime}=E_{3} E_{2}^{\gamma}, \quad E_{4}^{-1} E_{2} E_{4}=E_{2} E_{1}, \quad E_{3}^{-1} E_{2} E_{3}=E_{2} .
$$

Dopo ciò si osservi che l'elemento $E_{4}^{p}$ sta certamente in $K=G_{2} ;$ ma, giacchè il detto elemento è invertibile con $E_{4}$, esso sta in $G_{1}=H$.

Inoltre se è $p>2$, dalle (13) si ricava:

$$
E_{4}^{-p} E_{3} E_{4}^{p}=E_{3} E_{2}^{p}
$$

e siccome $E_{4}^{p}$ sta in $H$, deve essere $E_{2}^{p}=1$; quindi l'elemento $E_{3}^{p}$, che risulta allora invertibile con $E_{4}$, sta in $H$. Si ha dunque:

$$
E_{4}^{p}=E_{\mathrm{t}}{ }^{\alpha}, \quad E_{3}^{p}=E_{1}^{\beta}, \quad E_{2}^{p}=1, \quad E_{1}^{p}=1 .
$$

Le formole (13) e (14) definiscono sempre un gruppo $G^{2,2}$ tutte le volte che è $p>2$.

Infatti, considero i simboli già precedentemente definiti:

$$
e_{4}^{x} e_{2}^{y} e_{2}^{z} e_{1}^{t}
$$

e, come mi consigliano le (14), chiamo eguali due tali simboli quando si possono dedurre l'uno dall'altro facendo variare ordinatamente gli esponenti di $p k_{1}, p k_{3}, p k_{2}, h_{1}$, purchè sia:

$$
\alpha k_{4}+\beta k_{3}+h_{1} \equiv 0 \quad(\bmod p)
$$

inoltre, per andare poi d'accordo con le (13), pongo per definizione:

essendo:

$$
\left(e_{4}^{x^{\prime}} e_{3}^{y^{\prime}} e_{2}^{z^{\prime}} e^{t^{\prime}}\right)\left(e_{4}^{x^{\prime \prime}} e_{3}^{y^{\prime \prime}} e_{2}^{z^{\prime \prime}} e_{1}^{t^{\prime}}\right)=e_{4}^{x^{\prime \prime \prime}} e_{3} y^{y^{\prime \prime \prime}} e_{2}{ }^{z^{\prime \prime \prime}} e_{1}^{t^{\prime \prime \prime}}
$$

$$
\begin{gathered}
x^{\prime \prime \prime}=x^{\prime}+x^{\prime \prime}, \quad y^{\prime \prime \prime}=y^{\prime}+y^{\prime \prime}, \quad z^{\prime \prime \prime}=z^{\prime}+z^{\prime \prime}+y^{\prime} x^{\prime \prime} \\
t^{\prime \prime \prime}=t^{\prime}+t^{\prime \prime}+z^{\prime} x^{\prime \prime}+y^{\prime}\left(\begin{array}{c}
x^{\prime \prime} \\
2
\end{array}\right) .
\end{gathered}
$$

Poste queste definizioni e chiamando $A, B, C$ tre qualunque elementi come $e_{1}^{x} e_{3}^{y} e_{z}^{z} e^{t}{ }_{1}$, quando si vuole stabilire che tutte le volte che è $A=B$ è an- 
che $A C=B C$, bisogna tenere conto della condizione $p>2$; e supponendola verificata, le dette definizioni dànno origine ad un gruppo che è rappresentato in $\Gamma$ con un $G_{4},{ }^{2,2}$, e facendo corrispondere ai simboli:

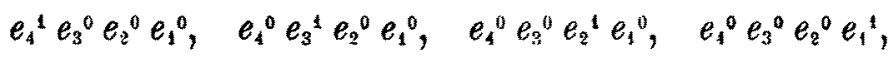

ordinatamente gli elementi $E_{4}, E_{3}, E_{2}, E_{1}$, questi elementi verificano le relazioni (13) e (14).

14. Ponendo a fondamento le dette relazioni, la serie canonica:

$$
G_{1}^{2,2}, G_{3}, G_{2}, G_{1},
$$

è perfettamente determinata nel senso che, se:

$$
\mathbf{F}_{4}, \mathbf{E}_{3}, \mathbf{E}_{2}, \mathbf{E}_{1},
$$

è un'altra base canonica di $G_{4}^{q, 2}$, tale che l'operazione:

$$
T=\left(\begin{array}{llll}
E_{4}, & E_{3}, & E_{2}, & E_{1} \\
\boldsymbol{V}_{4}, & \mathbf{F}_{3}, & \mathbf{F}_{2}, & \mathbf{F}_{1}
\end{array}\right)
$$

non alteri la forma delle (13) e (14), la detta base definisce la stessa serie canonica.

Ponendo dunque, nella più generale maniera:

$$
\mathbf{E}_{1} \equiv E_{4}^{u} E_{3}^{w}, \quad \mathbf{E}_{3} \equiv E_{3}^{s}, \quad\left(\bmod G_{2}\right)
$$

affinchè si conservino le formole (13) si deve scegliere $\mathbf{E}_{2} \equiv E_{2} u s\left(\bmod G_{1}\right)$ ed $\mathbf{k}_{1}=E_{1}^{u^{2} s}$; allora, per non contraddire alla definizione di $\mathbf{E}_{1}$, bisogna supporre $u$ ed $s$ primi con $p$.

Se dopo avere definita in tal modo la nuova base $\mathbf{k}_{1}, \mathbf{E}_{3}, \mathbf{E}_{2}, \mathbf{E}_{1}$, si fa l'operazione che porta la detta base in $E_{4}, E_{3}, E_{2}, E_{1}$ e si chiamano $\alpha_{1}$ e $\beta_{1}$ i valori trasformati di $\alpha$ e $\beta$, si trova facilmente:

$$
u^{2} s \alpha_{1} \equiv \alpha u+\beta v, \quad u^{2} \beta_{1} \equiv \beta, \quad(\bmod p),
$$

purchè si supponga $p>3$ e si osservi che, in virtù delle (13), si ha:

$$
\left(E_{4} E_{3}\right)^{n}=E_{4}^{n} E_{3}^{n} E_{2}^{\left(\begin{array}{c}
n \\
2
\end{array}\right)} E_{4}^{\left(\begin{array}{l}
n \\
3
\end{array}\right)} \text {. }
$$

Sia, in prima ipotesi, $\beta=0$; allora, se non ̀̀ $\alpha=0$, scegliendo $u=1$, $s=\alpha$, ottengo $\alpha_{1} \equiv 1(\bmod p)$.

Sia, in econda jpotesi, $\beta$ primo con $p$ : allora, posso scegliere $v$ in modo che sia $\alpha_{1} \equiv 0(\bmod p)$; inoltre, se $\beta$ è un residuo quadratico di $p$, posso de- 
terminare $u$ in modo che $\operatorname{sia} \beta_{1} \equiv 1(\bmod j)$; e se $\beta$ non è residuo quadratico di $p$, essendo $\varepsilon$ una qualunque radice primitiva di $p$, posso determinare $u$ in modo che sia $\beta_{i} \equiv \varepsilon(\bmod p)$.

Esistono dunque, se è $p>3$, quattro gruppi $G_{4}{ }^{2,2}$, i quali sono definiti dalle (13) e dalle seguenti modificazioni delle formule (14):

\begin{tabular}{|c|c|c|}
\hline$E_{4}^{p}=1$ & $E_{3}^{\mu}=1$ & $E_{2}^{p}=1, \quad E_{1}^{p}=1$ \\
\hline$E_{i}^{p}=E_{1}$ & $E_{3}^{p}=1$ & $E_{2}^{p}=1, \quad E_{1}^{p}=1$ \\
\hline$E_{4}^{p}=1$ & $E_{3}^{p}=E_{1}$ & $E_{2}^{p}=1, \quad E_{1}^{p}=1$ \\
\hline$E_{4}^{p}=1$ & $E_{3}^{y}=E_{1}^{\varepsilon}$ & $E_{z}^{p}=1, \quad E_{1}^{p}=1$ \\
\hline
\end{tabular}

Il divisore Abeliano $G_{3}$ dei primi due gruppi è un $G_{3}{ }^{0,3}$ e quello degli ultimi due è un $G_{3}{ }^{0,2}$.

15. Si supponga ora $p=3$.

Giacchè due qualunque operazioni $T$ appartenenti alla classe definita dalle congruenze (15) per dati valori di $u, v, s$, producono lo stesso effetto sopra i numeri $\alpha$ e $\beta$ che figurano nelle formule (14), io ritengo eguali tutte le operazioni appartenenti alla detta classe e quest'unica operazione la rappresento col simbolo:

$$
\begin{array}{ll:l}
u & v & . \\
0 & s & 1
\end{array}
$$

Tutte le possibili operazioni così definite costituiscono, nel presente caso, un gruppo di grado 12; ma, giacchè l'operazione:

$$
\mid \begin{array}{rr}
-1 & 0 \\
0 & -1
\end{array}
$$

non cambia a e $\beta$, jo la posso ritenere eguale all'elemento identico del detto gruppo e quindi il grado di questo si riduce a 6 . Il gruppo di grado 6, che qui si presenta, è isomorfo a quello di cui ho discorso nel $n^{\circ} 9$ e si può generare mediante le due operazioni:

$$
\begin{array}{ll}
1 & 0 \\
0 & 2
\end{array}, \quad, \begin{array}{ll}
1 & 1 \\
0 & 1
\end{array}
$$

che sono rispettivamente di secondo e terzo ordine. 
La prima di queste operazioni cambia $\alpha$ in $2 \alpha$ e la seconda cambia $\alpha$ in $\alpha+\beta+1$; ma, nè l'una nè l'altra alterano $\beta$. Dunque, se $\beta$ è 0 od 1 , posso sempre supporre $\alpha=0$, e se è $\beta=2$, posso ridurre il caso di $\%=2$ al caso di $\alpha=1$.

Esistono quindi, anche quando è $p=3$, quattro gruppi $\mathrm{G}_{4}^{2,2} \mathrm{i}$ quali corrispondono alle seguenti modificazioni delle formole (14):

$$
\begin{array}{ll}
E_{1}^{3}=1, \quad E_{3}^{3}=1, \quad E_{2}^{3}=1, \quad E_{1}^{3}=1 \\
E_{1}^{3}=1, \quad E_{3}^{3}=E_{1}, \quad E_{2}^{3}=1, \quad E_{1}^{3}=1 \\
E_{4}^{3}=1, \quad E_{3}^{3}=E_{1}^{2}, \quad E_{2}^{3}=1, \quad E_{1}^{3}=1 \\
E_{4}^{3}=E_{1}, \quad E_{3}^{3}=E_{1}^{2}, \quad E_{2}^{3}=1, \quad E_{1}^{3}=1
\end{array}
$$

Soltanto per il primo di questi gruppi il divisore Abeliano $G_{3}$ è un $G_{3}^{y_{3}^{0,3}}$; per gli altri tre gruppi il detto divisore è un $G_{3}^{0.2}$.

16. Io considero finalmente il caso, finora escluso, di $p=2$, per il quale non sussistono le formole (14).

Le relazioni (13) dànno:

$$
E_{4}^{-2} E_{3} E_{1}^{2}=E_{3} E_{2}^{2} E_{1}
$$

0 siccome $E_{4}^{2}$ sta in $H$, deve essere $E_{2}^{2}=E_{1}$; allora, l'elemento $E_{3}^{2}$ che, a causa della prima delle dette relazioni, non risulta permutabile con $E_{4}$, è una potenza impari di $L_{2}^{\prime}$.

Le (14) debbono dunque essere sostituite con le formole:

$$
H_{1}^{2}=E_{1}^{\prime}, \quad E_{3}^{2}=E_{2}^{p}, \quad E_{2}^{2}=E_{1}, \quad E_{1}^{2}=1
$$

ed è facile vedere che, tutto le volte che $\beta$ è impari, queste formole e le (13) definiscono un gruppo di grado $2^{4}=16$ che è un $G_{4}{ }^{2,2}$.

Cambiando ora la base canonica e ponendo, nella maniera più generale,

$$
\mathbf{F}_{4}=K_{4} E_{3}^{u}, \quad F_{3}=E_{3},
$$

la corrispondente operazione. $T$ non altera $\beta$ ma cambia $\alpha$ in un numero $\alpha_{1}$ tale che:

$$
s \alpha_{1} \equiv \nu+\frac{\beta+1}{2} v, \quad(\bmod 2) .
$$

Annali di Matematica, tomo I. 
Dunque, se è $\beta=1$, si può sempre ritenere $\alpha=0$, ma, se è $\beta=3$, bisogna distinguere il caso di $\alpha=0$ dal caso di $\alpha=1$.

Esistono percio tre gruppi di grado 16 ehe sono $G_{1}^{2,2}$, i quali sono definiti dalle formole:

$$
\begin{array}{llll}
E_{1}^{2}=1, & E_{3}^{2}=E_{2}, & E_{2}^{2}=E_{1}, & E_{1}^{2}=1 \\
\hline E_{4}^{2}=1, & E_{3}^{2}=E_{2} E_{1}, & E_{2}^{2}=E_{1}, & E_{1}^{2}=1 \\
\hline E_{4}^{2}=E_{1}, & E_{3}^{2}=E_{2} E_{1}, & E_{2}^{2}=E_{1}, & E_{1}^{2}=1 \\
\hline
\end{array}
$$

insieme alle formole (13).

Per questi tre gruppi il divisore Abeliano $G_{3}$ è ciclico.

17 Io riepilogo brevemente $\mathrm{i}$ risultati ottenuti nel presente paragrafo.

Esistono soltanto cinque gruppi di grado $p^{3}$, dei quali: tre sono gruppi $G_{3}{ }^{0}$ e gli altri due sono gruppi $G_{3}{ }^{1,2}$.

Esistono soltanto quattordici gruppi di grado $2^{4}=16$, dei quali: cinque sono gruppi $G_{4}^{0}$, tre sono gruppi $G_{4}{ }^{1,2}$, tre sono gruppi $G_{4}^{1,3}$ ed i rimanenti tre sono gruppi $G_{4}^{2,2}$.

Per tutti i valori del numero primo $p>2$ esistono soltanto quindici gruppi di grado $p^{4}$, dei quali: einque sono gruppi $G_{4}{ }^{0}$, tre sono gruppi $G_{4}{ }^{1,2}$, tre sono gruppi $G_{4}^{1,3}$ ed i rimanenti quattro sono gruppi $G_{4}^{*, 2}$.

Le formole di composizione di tutti questi gruppi sono rappresentate nella seguente tabella:

\section{Gruppi di grado $p^{3}$.}

I. Gruppi $G_{3}^{n}\left\{\left[p^{3}\right],\left[p^{2}\right][p],[p][p][p]\right\}$.

II. Gruppi $\left.G_{3}^{1,2} \mid E_{3}^{-1} E_{2} E_{3}=E_{2} E_{1}, H\left(E_{1}\right)\right\}$.

\begin{tabular}{c|c|c|c|}
\hline$E_{3}^{p}$ & $E_{2}^{p}$ & $E_{1}^{p}$ & \\
\hline 1 & 1 & 1 & $p \geqq 2$ \\
\hline 1 & $E_{1}$ & 1 & $p>2$ \\
\hline$E_{1}$ & $E_{1}$ & 1 & $p=2$ \\
\hline
\end{tabular}


Gruppi di grado $p^{4}$.

I. Gruppi $G_{i}^{0}\left|\left[p^{4}\right],\left[p^{3}\right][p],\left[p^{2}\right]\left[p^{2}\right],\left[p^{2}\right][p][p],[p][p][p][p]\right|$. II. Gruppi $G_{1}{ }^{1}\left\{E_{4}{ }^{-1} E_{3} E_{4}=E_{3} E_{1}, H\left(E_{2}, E_{1}\right)\right\}$.

\begin{tabular}{|c|c|c|c|c|c|c|c|} 
& $p \geq 2$ & $p \geqq 2$ & $p \geq 2$ & $p \geqq 2$ & $p \geqq 2$ & $p>2$ & $p=2$ \\
\hline$E_{4}^{p}$ & 1 & 1 & $E_{1}$ & 1 & 1 & 1 & $E_{1}$ \\
\hline$E_{3}^{p}$ & $J_{2}$ & $E_{2}^{\prime}$ & $E_{2}$ & 1 & 1 & $E_{1}$ & $E_{1}$ \\
\hline$E_{2}^{p}$ & $E_{1}$ & 1 & 1 & $E_{1}$ & 1 & 1 & 1 \\
\hline$E_{1}^{p}$ & 1 & 1 & 1 & 1 & 1 & 1 & 1 \\
& & Gruppi $G_{4}^{1,2}$ & & & Gruppi $G_{4}^{1,3}$ & \\
\hline
\end{tabular}

III. Gruppi $G_{4}{ }^{2}=G_{4}^{2.2},\left\{H\left(E_{1}\right)\right\}$. $E_{4}^{-1} E_{3} E_{4}=E_{3} E_{2}, \quad E_{4}^{-1} E_{2} E_{4}=E_{2} E_{1}, \quad E_{3}^{-4} E_{2} E_{3}=E_{2}$

\begin{tabular}{|c|c|c|c|c|c|c|c|c|}
\hline & $p \geqq 3$ & $p>3$ & $p \geqq 3$ & $p \geqq 3$ & $p=3$ & $p=2$ & $p=2$ & $p=2$ \\
\hline$E_{4}^{p}$ & 1 & $E_{1}$ & 1 & 1 & $E_{1}$ & 1 & 1 & $E_{1}$ \\
\hline$E_{3}^{n}$ & 1 & 1 & $E_{1}$ & $E_{1}^{\varepsilon}$ & $E_{1}^{2}$ & $E_{2}$ & $E_{2}^{-1}$ & $E_{2}^{-1}$ \\
\hline$E_{2}^{p}$ & 1 & 1 & 1 & 1 & 1 & $E_{1}$ & $E_{1}$ & $E_{1}$ \\
\hline$E_{1}^{p}$ & 1 & 1 & 1 & 1 & 1 & 1 & 1 & 1 \\
\hline
\end{tabular}

$\S$ III. I Gruppi $G_{5}{ }^{0}$ e $G_{5}{ }^{1}$.

18. Esistono sette gruppi Abeliani di grado $p^{5}$, i quali sono: un gruppo $G_{5}{ }^{0.1}$, due gruppi $G_{5}^{0,0,2}$, due gruppi $G_{\mathbf{5}}{ }^{1.9}$, un gruppo $G_{5}{ }^{0,4}$ ed un gruppo $G_{8}^{0,5}$. 
Mettendo in evidenza per ciascuno di questi sette gruppi gl'invarianti del sig. Frobenids, li rappresenterò nel seguente modo:

$$
G_{5}^{0,1}\left\{\left[p^{5}\right]\right\}, \quad G_{5}^{0,4}\left\{\left[p^{2}\right][p][p][p]\right\}, \quad G_{5}^{0.5}\{[p][p][p][p][p]\},
$$

$\left.G_{\mathbf{5}}{ }^{0,2} \mid\left\{p^{4}\right][p]\right\}, \quad G_{5}^{0,2}\left\{\left[p^{3}\right]\left[p^{2}\right]\right\}, \quad G_{5}^{0,3}\left\{\left[p^{3}\right][p \mid[p]\}, \quad G_{5}^{0,3} \mid\left[p^{2}\right]\left[p^{2} \mid[p]\right\}\right.$.

Il gruppo $G_{5}{ }^{0,1}$ è il gruppo ciclico di grado $p^{5}$; il gruppo $G_{5}^{0,4}$ possiede $\frac{p^{4}-1}{p-1}-1$ divisori $G_{4}^{0,3}$ ed un solo divisore $G_{4}^{0,4}$, mentre tutti i divisori d'indice $p$ del gruppo $G_{5}^{0,5}$ sono gruppi $G_{4}^{0.4}$. Il primo dei due gruppi $G_{5}^{0,2}$ ha un solo divisore $G_{4}^{0,2}\left\{\left[p^{3}\right][p] !\right.$ e $p$ divisori $G_{4}^{0,4}$, mentre il secondo dei detti due gruppi ha $p$ divisori $G_{4}^{0,2}\left\{\left[p^{3}\right][p]\right\}$ ed un solo divisore $G_{4}^{0,2}\left\{\left[p^{2}\right]\left[p^{2}\right]\right.$.

Finalmente, il primo dei due gruppi $G_{5}^{03}$ ha un solo divisore $G_{4}^{0,3}$ e $p(p+1)$ divisori $G_{4}{ }^{02}\left\{\left[p^{3}\right][p]\right\}$, mentre tutti i divisori d'indice $p$ del secondo dei detti due gruppi sono: $p+1$ gruppi $G_{4}^{0.3}$ e $p^{2}$ gruppi $G_{4}^{1,2}\left\{\left[p^{2}\right] \mid p^{2}\right] \mid$.

Io mi limito a dimostrare soltanto quello che ho asserito relativamente al gruppo $\left[p^{2}\right]\left[p^{2}\right][p]$.

Scegliendo in modo conveniente tre elementi $E^{\prime \prime}, E^{\prime \prime}, E^{\prime}$ dei gradi $p^{2}$, $p^{2}, p$ rispettivamente, un arbitrario elemento di un tale gruppo si può rappresentare con:

$$
E^{\prime \prime \mu} E^{\beta} E^{\prime \prime}
$$

dove $\alpha, \beta, \gamma$ sono tre interi presi ordinatamente rispetto ai moduli $p^{2}, p^{2}, p$.

I tre gruppi di grado $p^{4}$, costituiti rispettivamente dagli elementı dei seguenti tre tipi:

$$
E^{\prime \prime p} E^{\prime \beta} E^{\prime}, \quad E^{\prime \prime \alpha} E^{\prime p \beta} E^{\prime \gamma}, \quad E^{\prime \prime \alpha} E^{\prime \beta},
$$

sono indipendenti, perchè s'intersecano secondo il gruppo $K$ di grado $p^{2}$ costituito dagli elementi del tipo:

$$
l_{i}^{\prime \prime \prime} p^{\prime x} L^{\prime \prime p} p^{p} \text {. }
$$

Inoltre, un elemento qualunque del detto gruppo di grado $p^{2}$ è la $p^{m a}$ potenza di un elemento del gruppo principale e quiudi questo non può avere più di tre divisori indipendenti d'indice $p$ : dungue il gruppo principale $\left[p^{\circ}\right]\left[p^{2}\right][p]$ è un $\left(x_{5}^{0,3}\right.$.

Tutti gli elementi di grado $p$ di questo gruppo formano jl gruppo $C_{3}{ }^{3.3}$ costituito dagli elementi del tipo:

$$
E^{\prime \prime \prime} E^{\prime \prime} E^{\prime \beta} E^{\prime}
$$


Dei divisori d'indice $p$ del gruppo principale, quelli che contengono $G_{3}{ }^{0,3}$ sono necessariamente gruppi $G_{4}^{0,3}$ e, pensando al gruppo complementare di $G_{3}{ }^{0,3}$ nel detto gruppo principale, si vede subito che il loro numero è $p+1$.

Inoltre, ogni divisore $G_{4}{ }^{0.3}$ del gruppo principale contiene evidentemente $G_{3}{ }^{03}$ : dunque il detto gruppo possiede $p+1$, e non più, divisori $G_{4}{ }^{0.3}$.

Ciò posto, i rimanenti $p^{2}$ divisori d'indice $p$ sono necessariamente gruppi $\left.\left.\mathrm{G}_{4}^{0,2}: \mid p^{2}\right]\left[p^{2}\right]\right\}$.

19. I gruppi $G_{5}{ }^{1}$ possono essere o gruppi $G_{5}^{1,2}$ o gruppi $G_{5}^{1,3}$, oppure gruppi $i_{\mathrm{s}}{ }^{1,4}$, ed io mostrerò che effettivamente esistono gruppi in ognuna di queste classi.

Sia:

$$
G_{5}{ }^{1}, \quad G_{1}, \quad G_{3}, \quad G_{2}, G_{1}
$$

una serie canonica di un gruppó $G_{5}{ }^{1}$ seelta in modo tale che sia $G_{3}=H$, ed:

$$
E_{5}, \quad E_{4}, \quad E_{3}, \quad E_{2}, \quad E_{1}
$$

una base canonica relativa alla detta serie.

Giacchè la potenza $J_{4}^{\prime \prime}$ sta in $H$, l'elemento $E_{3}^{\prime}$, che figura nella relazione $E_{5}^{-1} E_{4} E_{5}=E_{4} E_{*}^{\prime}$, è di grado $p$ ed io lo posso assumere come elemento $E_{1}$; quindi si ha:

$$
E_{\mathrm{5}}-1 E_{1} E_{5}^{\prime}=E_{4} E_{1}^{\prime}
$$

Se il gruppo $H$ è ciclico, scegliendo opportunamente l'elemento $E_{3}$, posso scrivere:

$$
E_{\overline{0}}^{p}=E_{3}^{a}, \quad E_{4}^{p}=E_{3}^{\beta}, \quad E_{3}^{p}=E_{2}, \quad E_{2}^{p}=E_{1}, \quad E_{1}^{p}=1
$$

e si può facilmente dimostrare che, qualunque siano gl' interi a e $\beta$, le formole (1) e (2) definiscono un gruppo $G_{5}{ }^{1}$.

Ciò posto, si osservi che l'operazione:

$$
\left(\begin{array}{ccccc}
E_{5}, & E_{4}, & E_{3}, & E_{2}, & E_{1} \\
E_{5} E_{3}^{\prime \prime}, & E_{4} E_{3}^{h}, & E_{3}, & E_{2}, & E_{1}
\end{array}\right)
$$

cambia $\alpha$ e $\beta$ rispettivamente in $\alpha+h p$ e $\beta+h p$; dunque, se $\alpha$ e $\beta$ sono multipli di $p$, si può supporre $\alpha=\beta=0$.

Se uno dei due numeri $\alpha, \beta$ è primo con $p$, io suppongo che sia $\beta$ primo con $p$ giacchè, in caso contrario, scambio $\alpha$ in $\beta$ mediante l'operazione:

$$
\left(\begin{array}{lllll}
E_{5}, & E_{4}, & E_{3}, & E_{2}, & E_{1}^{3} \\
E_{4}^{-1}, & E_{5}^{-1}, & E_{3}^{-1}, & E_{2}^{-1}, & E_{1}^{-1}
\end{array}\right) ;
$$


poi, eseguendo la trasformazione:

$$
\left(\begin{array}{lllll}
E_{5}, & E_{1}, & E_{3}^{\beta}, & E_{2}, & E_{1} \\
E_{5}^{\beta}: & E_{4}, & E_{3}^{\beta}, & E_{2}^{\beta}, & E_{1}^{\beta}
\end{array}\right),
$$

mi riduco al caso di $\beta=1$. Dopo ciò, chiamando $E_{5}^{*}$ l'elemento $E_{5} E_{4}^{-\alpha}$, si vede facilmente che è sempre lecito ritenere $\alpha=0$

Esistono quindi due soli gruppi $G_{5}{ }^{1}$, che hanno il divisore invertibile ciclico, i quali sono definiti dalla (1) e dalle formole:

$$
\begin{aligned}
& E_{5}^{p}=1, \quad E_{4}^{p}=1, \quad E_{3}^{p}=E_{2}, \quad E_{2}^{p}=E_{1}, \quad E_{1}^{p}=1 \\
& E_{5}^{p}=1, \quad E_{4}^{p}=E_{3}, \quad E_{3}^{p}=E_{2}, \quad E_{2}^{p}=E_{1}, \quad E_{1}^{p}=1
\end{aligned}
$$

Il primo di questi gruppi è un $G_{5}^{4,3}$ e fra tutti i suoi divisori d'indice $p$, i quali s'intersecano in $G_{2}$, ve ne sono $p^{2}$ non Abeliani; il secondo gruppo è un $G_{5}{ }^{1.2}$ e tutti i suoi $p+1$ divisori d'indice $p$ sono Abeliani.

20. Si supponga ora che il gruppo $H=G_{3}$ sia un $G_{3}{ }^{0,2}$.

In questo caso, scegliendo come gruppo $G_{2}$ l'unico divisore non ciclico di grado $p^{2}$ che possiede $H$, due ipotesi sono possibili rispetto all'elemento $E_{3}^{p}$, che è necessariamente di grado $p: 0$ questo elemento sta in $G_{1}$ oppure è fuori di $G_{1}$. Nella prima ipotesi posso supporre $E_{3}^{p}=E_{3}$, e nella seconda ipotesi pongo $E_{3}^{p}=E_{2}$ : si hanno in corrispondenza ai due casi le due serie di formole:

$$
\begin{aligned}
& E_{5}^{p}=E_{3}^{\alpha} E_{2}^{\beta}, \quad E_{4}^{p}=E_{3}^{\gamma} E_{2}^{\delta}, \quad E_{3}^{p}=E_{1}, \quad E_{2}^{p}=1, \quad E_{1}^{p}=1 \\
& E_{5}^{p}=E_{3}^{\alpha} E_{1}^{\beta}, \quad E_{4}^{p}=E_{3}^{\gamma} E_{1}^{\delta}, \quad E_{3}^{p}=E_{2}, \quad E_{2}^{p}=1, \quad E_{1}^{p}=1 .
\end{aligned}
$$

Io dimostro ora che le (3) e la (1) definiscono sempre un gruppo $G_{5}{ }^{4}$ qualunque siano gl' interi $\alpha, \xi, \%, \delta$, e in un modo perfettamente analogo si pud dimostrare che anche le (4) e la (1) definiscono, in ogni caso, un gruppo $C_{5}{ }_{5}^{2}$.

Si considerino i simboli che si ottengono da:

$$
e_{5}^{x} e_{4}^{y} e_{3}^{z} e_{2}^{t} e_{1}^{x w}
$$

attribuendo ad $x, y, z, t, w$ arbitrari valori interi, e si chiamino eguali due tali simboli quando si ottengono l'uno dall'altro facendo variare $\mathrm{i}$ detti interi 
ordinatamente di $p k_{5}, p k_{4}, h_{3}, h_{2}, h_{1}$, purchè sia:

$$
\begin{aligned}
\alpha k_{5}+\gamma k_{4}+h_{3}+p h_{1} & \equiv 0, \quad\left(\bmod p^{2}\right) \\
\beta k_{5}+2 k_{4}+h_{2} & \equiv 0, \quad(\bmod p) .
\end{aligned}
$$

Dietro questa convenzione si ottengono soltanto $p^{5}$ simboli distinti.

Ora, ponendo per definizione:

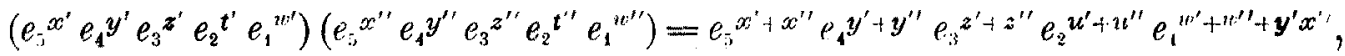

si può subito verificare che, rispetto a questa legge di composizinne, i simboli considerati costituiscono un gruppo $P_{5}$. Se si rappresenta questo gruppo in J' facendo corrispondere ai cinque elementi di $P_{5}$ :

$$
e_{3}{ }^{4} e_{4}{ }^{0} e_{3}{ }^{0} e_{2}{ }^{0} e_{1}{ }^{0}, \quad e_{5}{ }^{0} e_{4}{ }^{1} e_{3}{ }^{10} e_{2}{ }^{0} e_{1}{ }^{0}, \ldots, \quad e_{5}{ }^{0} e_{4}{ }^{0} e_{3}{ }^{0} e_{2}{ }^{0} e_{1}{ }^{1} \text {, }
$$

ordinatamente, i cinque elementi $E_{5}, E_{4}, E_{2}, E_{2}, E_{1}$ di $\mathrm{r}$, questi elementi costituiscono una base canonica di un $\left(_{x_{5}}^{1}\right.$ e verificano le relazioni (3) ed (1).

Io non ripeterò in seguito un simile ragionamento, che deve essere oramai familiare al lettore: mi limiterò solamente ad asserire, quando si presenta il caso, che una data serie di relazioni definiscono un gruppo di una data classe.

Riferendomi alle formole (3), se i numeri a e $\gamma$ sono multipli di $p$, siccome la trasformazione:

$$
\left(\begin{array}{ccccc}
E_{3} & E_{1} & E_{3} & E_{2} & E_{1} \\
E_{\mathrm{x}} E_{3}^{h} & E_{4} E_{3}^{k} & E_{3} & E_{2} & E_{1}
\end{array}\right)
$$

cambia $\mathrm{i}$ detti numeri rispettivamente in $\alpha+h p$ e $\gamma+k p$, posso supporre $\alpha=\gamma=0$. Ciò posto, se uno dei due numeri $\beta$, ò ̀̀ primo con $p$, ritengo, senza nuocere alla generalità, $\partial$ primo con $p$ e pongo a definizione dell'elemento $E_{2}$ la relazione $F_{4}{ }^{p}=E_{2}$; allora, cambiando $E_{5} E_{1}{ }^{-\beta}$ in $E_{5}$, si vede facilmente che si può assumere $\beta=0$. Ottengo quindi due gruppi $G_{5}{ }^{1}$ definiti dalla (1) e dalle relazioni:

$$
\begin{array}{ll}
E_{5}^{p}=1, \quad E_{4}^{p}=E_{2}, \quad E_{3}^{p}=E_{1}, \quad E_{2}^{p}=1, \quad E_{1}^{p}=1 \\
E_{5}^{p}=1, \quad E_{4}^{p}=1, \quad E_{3}^{p}=E_{1}, \quad E_{2}^{p}=1, \quad E_{1}^{p}=1
\end{array}
$$


Il primo di questi gruppi è un $C_{i}^{4,3}$ ed il relativo gruppo $K$ coincide con $G_{2}$, mentre il secondo è un $G_{5}^{1.4}$ ed il relativo gruppo $K$ coincide con $G_{1}$.

Se uno dei due numeri $\alpha, y$ è primo con $p$, posso supporre che sia $\gamma$ primo con $p$ : allora, facendo la trasformazione:

$$
\left(\begin{array}{llrrr}
E_{5}^{\prime}, & E_{4}^{\prime}, & E_{3}, & E_{2}^{\prime}, & E_{1} \\
E_{5}^{*}, & E_{4}^{\prime}, & E_{3}^{*} E_{2}^{\delta}, & E_{2}, & E_{1}^{\gamma}
\end{array}\right)
$$

ottengo $E_{4}^{p}=E_{3}$. Ciò posto, chiamando $F_{5}$ l'elemento $E_{5} E_{4}^{-a}$, si vede facilmente che si può assumere $\alpha=0$; allora, se è $\beta$ primo con $p$, pongo a definizione dell' elemento $E_{2}$ la relazione $E_{5}^{p}=E_{2}$.

Ottengo quindi due nuovi gruppi $G_{5}{ }^{4}$ definiti dalle (1) e dalle formole:

$$
\begin{aligned}
& E_{5}^{p}=E_{2}, \quad E_{4}^{p}=E_{3}, \quad E_{3}^{p}=E_{1}, \quad E_{2}^{p}=1, \quad E_{1}^{p}=1 \\
& E_{5}^{p}=1, \quad E_{4}^{p}=E_{3}, \quad E_{3}^{p}=E_{1}^{\prime}, \quad E_{2}^{p}=1, \quad E_{1}^{p}=1
\end{aligned}
$$

11 primo di questi gruppi è un $\left(_{r_{5}}^{1,2}\right.$ ed il relativo gruppo $K$ coincide con $H$, mentre il secondo è un $C_{s}^{*}$ ed il relativo gruppo $K$ coincide onl gruppo ciclico generato dall'elemento $F_{3}$.

21. Riferendomi alle formole (4), siano, in prima ipotesi, $\alpha$ e $y$ multipli di $p$ : in tale caso posso supporre come prima $\alpha=y=0$. Cuò posto, se uno dei due numeri $\beta$, $\partial$ è primo con $p$, in suppongo " primo con $p$; poi, facendo la trasformazione:

$$
\left(\begin{array}{lllll}
E_{5}^{\prime}, & E_{4}, & E_{3}, & E_{2}^{\prime}, & E_{1} \\
E_{5}^{\delta}, & E_{4}, & E_{3}, & E_{2}, & E_{1}^{\phi}
\end{array}\right)
$$

mi riduco al caso di $\delta=1$. Allora, supponento $p=2$ e chiamando $F$ l'ele mento $E_{s} E_{4}^{-r}$, posso supporre $\beta=0$.

Ottengo verciò due gruppi i quali, nell'attuale ipotesi di $p>2$, sono distinti, e questi gruppi sono definiti dalla (1) e dalle scguenti modificazioni delle formole (4):

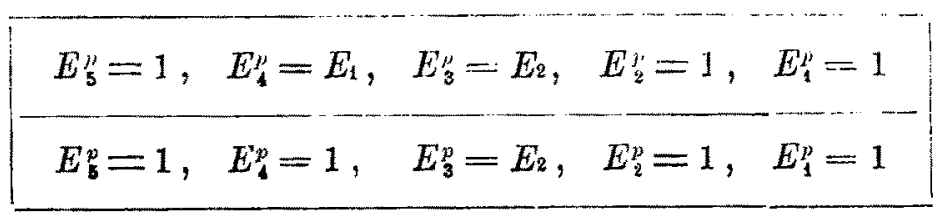


Entrambi questi gruppi appartengono alla classe dei gruppi $G_{5}^{1,3}$ ed i relativi gruppi $K$ coincidono con $G_{2}$.

Se invece è $p=2$, si vede subito, pensando alla (1), che il caso di $E_{\mathbf{s}}=E_{1}$, $E_{4}=E_{1}$ non è riducibile ad alcun altro caso, mentre poi tutti questi altri casi sono riducibili al caso di $F_{5}=1, E_{4}=1$.

Dunque ottengo due gruppi $C_{5}{ }^{1}$ di grado $2^{5}=32$, i quali sono definiti dalle formole:

$$
\begin{array}{ll}
E_{5}^{2}=E_{1}, \quad E_{1}^{2}=E_{1}, \quad E_{3}^{2}=E_{2}, \quad E_{2}^{2}=1, \quad E_{1}^{2}=1 \\
E_{5}^{2}=1, \quad E_{1}^{2}=1, \quad E_{3}^{2}=E_{2}, \quad E_{2}^{2}=1, \quad E_{1}^{2}=1
\end{array}
$$

insieme alla formula (1).

Entrambi questi gruppi sono gruppi $G_{5}^{1,3}$ e, in ognuno di essi, il divisore $K$ coincide con $G_{2}$.

Sia, in seconda ipotesi, uno dei due numeri $a, \gamma$ primo con $p$ e precisamente si supponga $\gamma$ primo con $p$. Allora, facendo la trasformazione:

$$
\left(\begin{array}{ccccc}
E_{5}^{\prime} & E_{1}^{\gamma} & E_{3} & E_{2} & E_{1} \\
E_{5} & E_{1}^{*} & E_{3}^{\gamma} E_{2}^{\gamma} & E_{2}^{\gamma} & E_{1}
\end{array}\right)
$$

ottengo $E_{4}^{p}=E_{3}$. Ciò posto, chiamando $E_{\mathrm{s}}$ l'elemento $E_{5} E_{4}^{-a}$, posso ritenere $\alpha=0$; poi, se non è $\beta$ multiplo di $p$, facendo la trasformazione:

$$
\left(\begin{array}{ccccc}
E_{5} & E_{4} & E_{3} & E_{2} & E_{1} \\
E_{5} & E_{4}^{\beta} & E_{3}^{\beta} & E_{2}^{\beta} & E_{1}^{\beta}
\end{array}\right)
$$

mi riduco al caso di $\beta=1$.

Ottengo perciò due gruppi $C_{5}{ }^{1}$, che sono definiti dalla (1) e dalle formole:

$$
\begin{aligned}
& E_{5}^{p}=E_{1}, \quad E_{4}^{p}=E_{3}, \quad E_{3}^{p}=E_{2}, \quad E_{2}^{p}=1, \quad E_{1}^{p}=1 \\
& E_{5}^{p}=1, \quad E_{4}^{p}=E_{3}, \quad E_{3}^{p}=E_{2}, \quad E_{2}^{p}=1, \quad E_{1}^{p}=1
\end{aligned}
$$

Ognuno di questi due gruppi è un gruppo $G_{6}^{1^{1,2}}$ che ha il relativo divisore $K$ coincidente con $H$. 
22. To abbandono ora l'ipotesi fatta al principio del n. 20 e suppongo invece che il gruppo $H=G_{3}$ sia un $G_{3}{ }^{0,3}$.

In questo caso si può porre nella più generale maniera:

$$
E_{5}^{p}=E_{3}^{a} E_{2}^{p} E_{1}^{\alpha}, \quad E_{4}^{p}=E_{3}^{\gamma} E_{2}^{\delta} E_{1}^{\mu}, \quad E_{3}^{p}=1, \quad E_{2}^{p}=1, \quad E_{1}^{p}=1,
$$

e queste formole, insieme alla (1), definiscono sempre un gruppo $G_{s}{ }^{1}$ qualunque siano gl' interi $\alpha, \beta, \lambda, \%, \delta, \mu$.

Siano, in prima ipotesi, i quattro numeri $\alpha, \beta, \gamma, \delta$ multipli di $p$. Allora, se uno dei due numeri $\lambda, \mu$ è primo con $p$, io suppongo $\mu$ primo con $p$; poi, mediante la traformazione:

$$
\left(\begin{array}{lllll}
E_{5} & E_{4} & E_{3} & E_{2} & E_{1} \\
E_{\mathrm{s}}^{\mu} & E_{1} & E_{3} & E_{2} & E_{1}^{\mu}
\end{array}\right)
$$

mi riduco al caso di $\mu=1$. Ciò posto, se è $p=2$, chiamando $E_{5}$ l'elemento $E_{s} E_{4}^{-\lambda}$, si vede che è lecito ritenere $\lambda_{1}=0$.

Si hanno quindi due gruppi $G_{5}{ }^{1}$ in corrispondenza alle formole:

$$
\begin{array}{llll}
E_{5}^{n}=1, & E_{4}^{p}=E_{1}, \quad E_{3}^{p}=1, & E_{2}^{p}=1, & E_{1}^{p}=1 \\
E_{5}^{n}=1, \quad E_{4}^{p}=1, \quad E_{3}^{p}=1, & E_{2}^{p}=1, & E_{1}^{p}=1
\end{array}
$$

Questi due gruppi appartengono alla classe dei gruppi $G_{5}^{1,4}$ e, per ognuno di essi, il gruppo $K$ coincide con $G_{1}$.

Se è $p=2$, si vede facilmente che il caso di $E_{5}^{2}=E_{1}, E_{4}^{2}=E_{1}$ si trasforma sempre in sè stesso mediante le operazioni $T$, che lasciano inalterata la (1) e la forma delle (5), mentre qualunque altro caso è riducibile a quello in cui ̀̀ $E_{5}^{2}=1, E_{4}^{2}=1$.

Percid si hanno due gruppi $G_{5}{ }^{1}$ di grado 32 in corrispondenza alle due serie di formole:

$$
\begin{aligned}
& E_{\mathrm{s}}^{2}=E_{1}, \quad E_{1}^{2}=E_{1}, \quad E_{3}^{2}=1, \quad E_{2}^{2}=1, \quad E_{1}^{2}=1 \\
& E_{5}^{2}=1, \quad E_{1}^{2}=1, \quad E_{3}^{2}=1, \quad E_{2}^{2}=1, \quad E_{1}^{2}=1
\end{aligned}
$$

e questi due gruppi appartengono alla classe dei gruppi $G_{5}{ }^{1,4}$. 
Sia, in seconda ipotesi, uno dei numeri $\alpha, \beta, \gamma, \delta$ primo con $p$. In tal caso, senza nuocere alla generalità, si può ritenere che uno dei due numeri $\gamma, \delta$ sia primo con $p$ e quindi è lecito porre a definizione dell' elemento $E_{2}$ la relazione $E_{4}^{p}=E_{2}$; allora chiamando $E_{5}$ l'elemento $E_{8} E_{4}{ }^{-\beta}$, si vede che si può assumere $\beta=0$. Cì̀ posto, se $\alpha$ è primo con $p$, pongo a definizione dell'elemento $E_{3}$ la relazione $E_{5}^{p}=E_{3}$; in caso contrario, bisogna distinguere l'ipotesi di $\lambda$ primo con $p$ da quella in cui è $\lambda$ un multiplo di $p$; e, nella prima ipotesi, facendo la trasformazione:

$$
\left(\begin{array}{lllll}
E_{5}, & E_{4}, & E_{3}, & E_{2}, & E_{1} \\
E_{5}, & E_{1}^{\lambda}, & E_{3}, & E_{2}^{\lambda}, & E_{1}^{\lambda}
\end{array}\right),
$$

mi riduco al caso di $\lambda=1$.

Si hanno quindi tre gruppi $G_{5}{ }^{1}$ ehe sono definiti dalla (1) e dalle seguenti modificazioni delle formole (5):

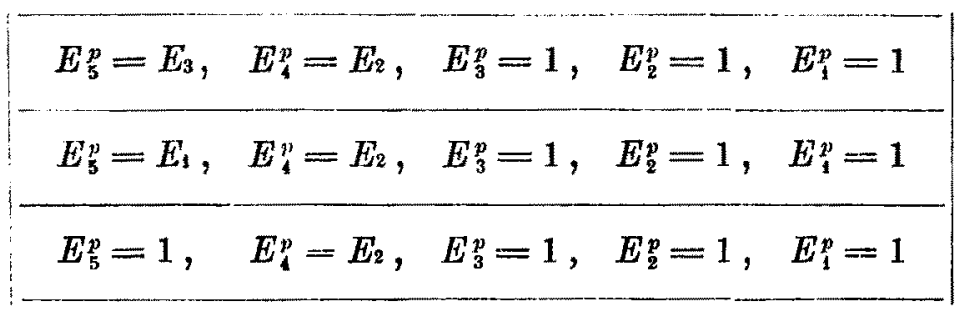

Il primo di questi gruppi è un $G_{5}{ }^{1,2}$, mentre gli ultimi due appartengono alla classe dei gruppi $\dot{G}_{5}^{1,3}$.

23. Riepilogando: qualunque sia il numero primo $p$, io ho trovato nel presente paragrafo ventidue gruppi di grado $p^{5}$.

Di questi ventidue gruppi, sette sono Abeliani e quindici sono gruppi $G_{\mathbf{s}}{ }^{1}$.

I quindici gruppi $G_{5}{ }^{1}$ trovati sono tutti i possibili gruppi $G_{5}{ }^{1}$ e sono: cinque gruppi $G_{5}^{1,2}$, sette gruppi $G_{5}^{1,3}$ e tre gruppi $G_{5}^{1,4}$.

I detti ventidue gruppi di grado $p^{5}$ sono brevemente rappresentati nella seguente tabella: 


\section{Gruppi $G^{0}{ }^{0}$.}

$\left[p^{5}\right], \quad\left[p^{2}\right][p][p][p], \quad[p][p][p][p][p]$, $\left[p^{4}\right][p], \quad\left[p^{3}\right]\left[p^{2}\right], \quad\left[p^{3}\right][p][p], \quad\left[p^{2}\right]\left[p^{2}\right][p]$.

II. Gruppi $G_{5}{ }^{1}$.

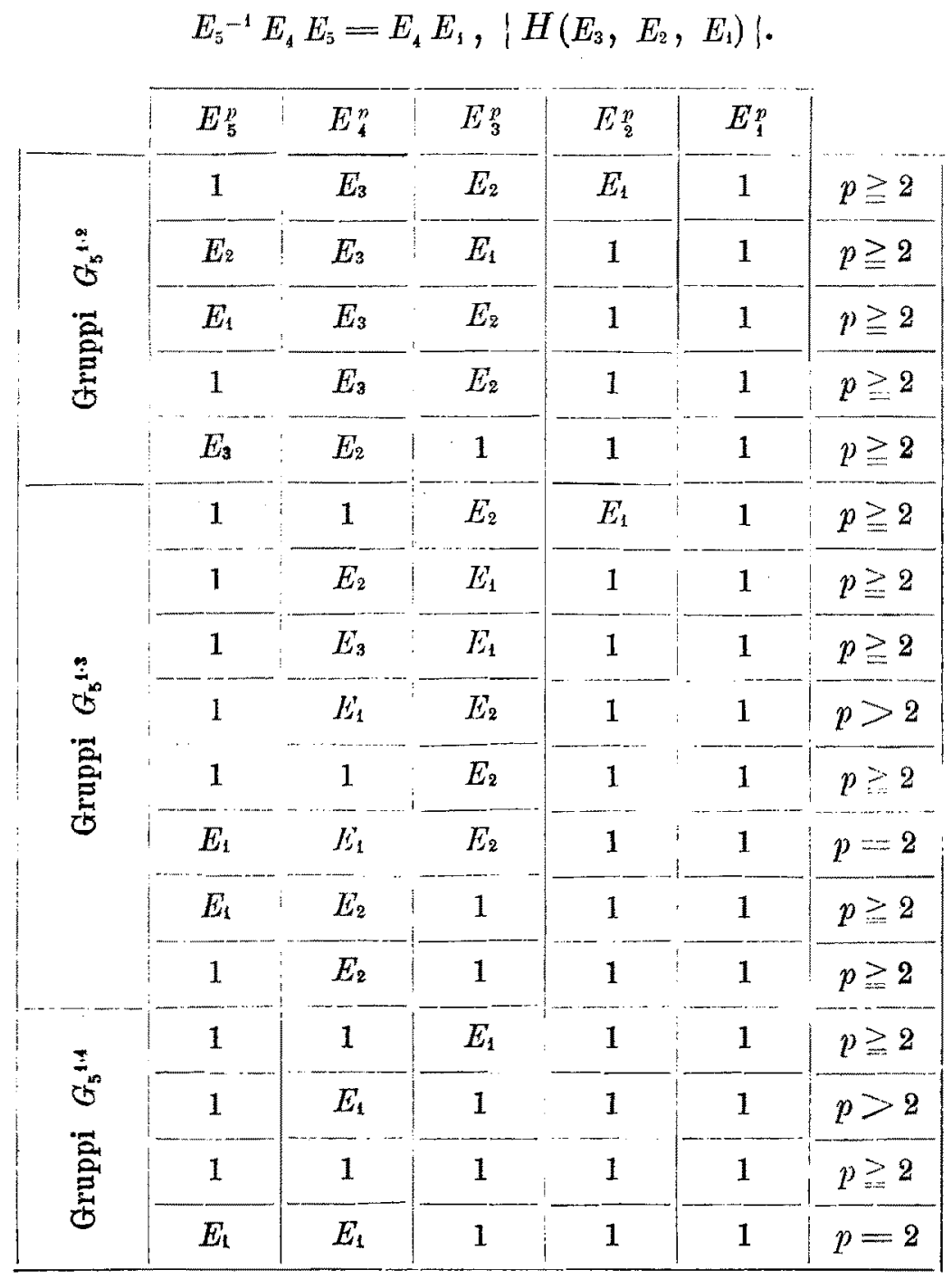




\title{
La composizione dei Gruppi finiti il cui grado è la quinta potenza di un nu- mero primo.
}

\author{
(Di G. Bagnera, a Palermo.)
}

PARTE SECONDA.

\begin{abstract}
$\S$ IV. I Gruppi $G_{5}^{2}$
che non posseggono alcun divisore Abeliano d'indice $p$.
\end{abstract}

24. I gruppi $G_{5}{ }^{2}$ possono essere o gruppi $G_{5}{ }^{2,2}$ o gruppi $G_{5}^{2,3}$ giacchè, come ora dimostrerò, non esistono gruppi $G_{5}{ }^{2,4}$.

Infatti, supposta l'esistenza di un gruppo $G_{5}{ }^{2,4}$, sia $E$ un elemento di grado $p$ generatore del gruppo $K$ di $G_{5}{ }^{2,4}$.

Siccome $E$ appartiene certamente ad $H$, il gruppo complementare $G_{5}{ }^{2,4} ; H$, di grado $p^{3}$, è Abeliano ed ha tutti i suoi elementi di grado $p$. Essendo $E^{\prime} H, E^{\prime \prime} H, E^{\prime \prime} H$ tre elementi generatori del detto gruppo complementare, si ha:

$$
E^{\prime-1} E^{\prime \prime} E^{\prime}=E^{\prime \prime} E^{\alpha}, E^{\prime-1} E^{\prime \prime \prime} E^{\prime}=E^{\prime \prime \prime} E^{\beta}, E^{\prime \prime-1} E^{\prime \prime \prime} E^{\prime \prime}=E^{\prime \prime \prime} E^{\gamma} .
$$

Giacchè il determinante :

$$
\left|\begin{array}{rrr}
0 & \alpha & \beta \\
-\alpha & 0 & \gamma \\
-\beta & -\gamma & 0
\end{array}\right|
$$

è nullo identicamente, riesce possibile determinare tre numeri interi $x, y, z$, non tutti multipli di $p$, tali che sia:

$$
\alpha y+\beta z \equiv 0,-\alpha x+\gamma z \equiv 0,-\beta x-\gamma y \equiv 0,(\bmod p) .
$$

Allora l' elemento $E^{\prime x} E^{\prime \prime y} E^{\prime \prime \prime z}$, che è fuori di $H$, risulta, in virtù delle (1), invertibile con ogni elemento di $G_{5}{ }^{2,4}$ e cid è assurdo. Annali di Matematica, tomo I. 
Stabilito ciò, io passo ora a dimostrare che, se è $p>2$, la potenza $p^{m a}$ di un elemento qualunque di un gruppo $G_{5}{ }^{2}$ appartiene al divisore invertibile $H$.

Sia, se è possibile, $E$ un elemento di $G_{5}^{2}$ tale che $E^{p}$ non appartenga ad $H$. Il gruppo di grado minimo, che contiene l'elemento $E$ ed il divisgre $H$, è Abeliano; quindi, non potendo coincidere con $G_{\mathrm{a}}{ }^{2}$, detto gruppo è un divisore $G_{4}$, d'indice $p$, di $G_{5}{ }^{2}$.

Si chiami $E^{\prime}$ un elemento di $G_{5}{ }^{2}$ fuori di $G_{4}$ e si ponga:

$$
E^{\prime-1} E E^{\prime}=E E^{\prime \prime}, \quad E^{\prime-1} E^{\prime \prime} E^{\prime}=E^{\prime \prime} H_{1}^{\prime \prime \prime} \text {. }
$$

L'elemento $E^{\prime p}$ appartiene certamente ad $H$; altrimenti il gruppo di grado minimo che contiene $E^{\prime}$ ed $H$ sarebbe un seçondo divisore Abeliano di $G_{5}{ }^{2}$; allora, l'intersezione di questi due divisori, che è di grado $p^{3}$, dovrebbe essere contenuta nel gruppo $H$ che, in un gruppo $G_{5}{ }^{2}$, è di grado $p^{2}$. L'elemento $E^{\prime \prime}$ appartiene al gruppo $K$ intersezione di tutti i divisori d'indice $p$ di $G_{\mathbf{5}}{ }^{2}$ e si può, in prima ipotesi, ammettere che $E^{\prime \prime}$ appartenga ad $H$. Allora dalla prima delle (2) si ricava:

$$
E^{\prime-p} E E^{\prime p}=E E^{\prime \prime p}, \quad E^{\prime-1} E^{p} E^{\prime}=E^{p} E^{\prime \prime p} ;
$$

quindi, giacchè $E^{\prime p}$ sta in $H$, risulta $E^{\prime \prime p}=1$. L'elemento $E^{p}$ è dunque invertibile con $E^{\prime}$ e perciò con ogni elemento di $G_{5}{ }^{2}$, il che contraddice alla definizione di $E$.

Sia, in seconda ipotesi, $E^{\prime \prime}$ fuori di $H$ e si pensi alla intersezione dei gruppi $K$ ed $H$. Se $K$ è di grado $p^{3}$, il gruppo $G_{5}{ }^{2}$ è un $G_{5}{ }^{2,2}$ e quindi $\left(\mathbf{n}^{\circ} 4\right) H$ è contenuto in $K$; se invece $K$ è di grado $p^{2}$, il gruppo $G_{53}{ }^{2} \grave{e ̀ ~ u n ~} G_{5}{ }^{2,3}$ di cui $K$ è divisore normale, e siccome detto divisore non coincide con $H$, esso contiene un sotto gruppo proprio di $H$. In ogni caso, si vede che la detta intersezione è un divisore d'indice $p$ di $K$; quindi $E^{\prime \prime p}$ ed $E^{\prime \prime \prime}$ sono elementi di questa intersezione e perciò di $H$.

Allora le formole (2) dànno:

$$
E^{\prime-p} E E^{\prime p}=E E^{\prime \prime p} E^{\prime \prime \prime}\left(\begin{array}{l}
p \\
2
\end{array}\right), E^{\prime-1} E^{\prime \prime p} E^{\prime}=E^{\prime \prime p} E^{\prime \prime \prime p}
$$

e quindi deve essere $E^{\prime / / p}=1, E^{\prime / p}=1$, purchè si pensi che ̀̀ $p>2$.

Dopo ciò, tenendo presente la prima delle (2), si vełe che Ep risulta invertibile con $E^{\prime}$ e con ogni elemento di $G_{5}{ }^{2}$ : dunque l'elemento $E$ is assurdo. 
25. Un gruppo $G_{5}{ }^{2}$ o contiene uno, ma soltanto uno, oppure nessuno divisore Abeliano d'indice $p$, ed io voglio anzitutto mettermi in quest' ultima ipotesi.

Se :

$$
G_{5}^{2}, \quad G_{1}^{4}, G_{3}, G_{2}, G_{1},
$$

è una serie canonica di composizione di un tale gruppo scelta in modo che $\operatorname{sia} G_{2}=H$, ed

$$
E_{\mathrm{s}}, \quad E_{4}, E_{3}, E_{2}, E_{1}
$$

è una base relativa alla detta serie, si ha:

$$
E_{5}^{-1} E_{4} E_{5}=E_{4} E_{3}^{\prime}, \quad E_{5}^{-1} E_{3} E_{5}=E_{3} E_{2}^{\prime}, \quad E_{4}^{-1} E_{3} E_{4}=E_{3}^{\prime} E_{2}^{\prime \prime} \text {. }
$$

Giacchè $E_{3}^{p}$ sta in $H$, dalle (3) risulta $E_{2}^{\prime p}=1, E_{2}^{\prime \prime p}=1$; inoltre gli elementi $E_{2}^{\prime}$ ed $E_{2}^{\prime \prime}$ non possono appartenere ad uno stesso gruppo ciclico, perchè, se fosse ad es. $E_{2}^{\prime \gamma}=E_{2}^{\prime \prime}$, l'elemento $E_{5}^{-\gamma} E_{4}$, che non sta in $G_{3}$, risulterebbe permutabile con $E_{3}$ e quindi, contrariamente all'attuale ipotesi, il gruppo $G_{5}^{2}$ ammetterebbe un divisore Abeliano d'indice $p$. Dunque $E_{2}^{\prime}$ ed $E_{2}^{\prime \prime}$ sono due elementi di grado $p$ capaci di generare il gruppo $G_{2}$.

Riguardo all' elemento $E^{\prime}{ }_{3}$, dico che esso è fuori di $G_{i}$; giacchè, se fosse $E_{3}^{\prime}=E_{2}^{\prime \alpha} E_{2}^{\prime \prime \beta}$, i due elementi $E_{5} E_{3}^{\beta}, E_{4} E_{3}^{-\alpha}$, in virtù delle (3), risulterebbero invertibili e quindi il gruppo $G_{5}^{2}$ ammetterebbe il divisore Abeliano d'indice $p$ generato dai detti due elementi e dagli elementi $E_{2}^{\prime}, E_{2}^{\prime \prime}$.

Il ragionamento precedente prova che, dati i due elementi $E_{5}, E_{4}$, si possono definire $E_{3}, E_{2}, E_{1}$ mediante le formole:

$$
E_{5}^{-1} E_{4} E_{8}=E_{4} E_{3}, \quad E_{5}^{-1} E_{3} E_{5}=E_{3} E_{2}, \quad E_{4}^{-1} E_{3} E_{4}=E_{3} E_{1} .
$$

Escludo subito il caso di $p=2$, perchè mediante le (4) si puð dimostrare che ogni gruppo $G_{5}^{2}$ di grado 32 ha necessariamente un divisore Abeliano d'indice 2.

Infatti, se un tale gruppo $G_{5}^{2}$ non ha un divisore Abeliano d'indice 2, debbono essere verificate le (4) e quindi deve essere:

$$
E_{5}^{-2} E_{4} E_{5}^{2}=E_{1} E_{3}^{2} E_{2}
$$

allora, se $E_{5}^{2}$ sta in $H$, risulta $E_{3}^{2}=E_{2}$. In questo caso, siccome le (4) dànno anche :

$$
E_{5}^{-1} E_{1}^{2} E_{5}=E_{4}^{2} E_{3}^{2} E_{1}=E_{4}^{2} E_{2} E_{1},
$$




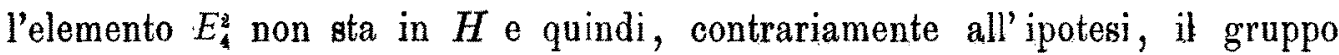
$G_{5}^{2}$ contiene il divisore Abeliano d'indiee 2 generato dagli elementi $E_{1}, E_{2}, E_{1}$.

Supponendo dunque $p>2$, dalle (4) facilmente si ricava:

$$
E_{5}^{-p} E_{4} E_{5}^{p}=E_{4} E_{3}^{p}
$$

e siccome $E_{5}^{p}$ sta $\left(\mathrm{n}^{\circ}{ }^{0} 24\right)$ in $H$, risulta $E_{3}^{p}=1$. Posso dunque scrivere:

$$
E_{5}^{p}=E_{2}^{a} E_{1}^{\beta}, \quad E_{4}^{p}=E_{2}^{\gamma} E_{1}^{\delta}, \quad E_{3}^{p}=1, \quad E_{2}^{p}=1, \quad E_{1}^{p}=1 ;
$$

e, tenendo presente che $E_{2}, E_{1}$ appartengono al divisore invertibile $H$, si dimostra col solito procedimento che, nell'ipotesi di $p>2$, qualunque siano gl'interi $\alpha, \beta, \gamma, \delta$, le formole (5) e (4) definiscono sempre un gruppo $G_{5}^{2}$. 亡 poi evidente che tutti i gruppi $G_{5}^{2}$ cosi definiti sono gruppi della classe $G_{5}{ }^{2,2}$.

26. Bisogna ora vedere come variano $\alpha, \beta, \gamma, \delta$ relativamente al gruppo delle operazioni :

$$
T=\left(\begin{array}{lllll}
E_{5} & E_{4} & E_{3} & E_{2} & E_{1} \\
\mathbf{E}_{5} & \mathbf{E}_{4} & \mathbf{E}_{3} & \mathbf{E}_{2} & \mathbf{E}_{1}
\end{array}\right)
$$

che lasciano inalterate le (4).

Ponendo:

$$
\mathbf{E}_{5} \equiv E_{5}^{u} E_{4}^{v}, \quad \mathbf{E}_{4} \equiv E_{5}^{r} E_{4}^{s}, \quad\left(\bmod G_{3}\right),
$$

affinchè la prima delle $(4)$ si conservi, bisogna prendere $\mathbf{E}_{3} \equiv E_{3}^{A}\left(\bmod G_{2}\right)$; quindi, per non contraddire alla definizione di $\mathbf{E}_{3}$, deve essere il determinante $\Delta=u s-v r$ primo con $p$; poi, affinchè si conservino le ultime due delle formole (4), deve essere:

$$
\mathbf{E}_{2}=E_{2}^{A u} E_{1}^{A v}, \quad \mathbf{E}_{1}=E_{2}^{A r} E_{1}^{A s} .
$$

D' altra parte, io osservo che le operazioni $T$ appartenenti alla classe definita dalle relazioni:

$$
\mathbf{E}_{5} \equiv E_{5}, \quad \mathbf{E}_{4} \equiv E_{1}, \quad\left(\bmod G_{3}\right),
$$

non alterano $\alpha, \beta, \gamma, \delta$, perchè allora, rammentando che è $p>2$, le (4) dànno :

$$
\mathbf{E}_{5}^{p}=E_{5}^{p}, \quad \mathbf{E}_{1}^{p}=E_{4}^{p}, \quad \mathbf{E}_{2}=E_{2}, \quad \mathbf{E}_{1}=E_{1} .
$$

Inoltre le dette operazioni costituiscono evidentemente un gruppo, che è divisore normale del gruppo di tutte le operazioni $T$ che conservano le (4); dunque, giacchè m'interessa studiare le variazioni di $\alpha, \beta, \gamma, \delta$, posso ritenere eguale all'operazione identica ogni operazione di detta classe. In questa 
ipotesi le (6) definiscono un' unica operazione che io rappresenterò con la notazione:

$$
\left|\begin{array}{ll}
u & v \\
r & s
\end{array}\right|
$$

Ora si osservi che le relazioni (4), dietro un facile calcolo, dànno:

$$
\left(E_{5}^{n} E_{4}^{k}\right)^{n}=E_{5}^{n h} E_{4}^{n k} E_{3}^{\left(\begin{array}{l}
n \\
2
\end{array}\right) h k} E_{2}^{\lambda} E_{1}^{\mu}
$$

dove per brevità si è ritenuto:

$$
\lambda=k\left\{h^{2}\left(\begin{array}{l}
n \\
3
\end{array}\right)+\left(\begin{array}{l}
n \\
2
\end{array}\right)\left(\begin{array}{l}
n \\
2
\end{array}\right)\right\}, \quad \mu=h\left\{2 k^{3}\left(\begin{array}{l}
n \\
3
\end{array}\right)+k^{2}\left(\begin{array}{l}
n \\
2
\end{array}\right)+\left(\begin{array}{l}
k \\
2
\end{array}\right)\left(\begin{array}{l}
n \\
2
\end{array}\right)\right\} .
$$

Dunque, supponendo $p>3$, in virtù delle (5) si ha:

$$
\left(E_{5}^{h} E_{4}^{k}\right)^{p}=E_{5}^{p h} E_{4}^{p k}=E_{2}^{h \alpha+k \gamma} E_{1}^{h \beta+k \delta} .
$$

Ciò posto, chiamando $\alpha_{1}, \beta_{1}, \gamma_{1}, \delta_{1} \mathrm{i}$ valori che assumono rispettivamente $\alpha, \beta, \gamma, \delta$ dopo avere eseguita la trasformazione:

$$
\left|\begin{array}{ll}
u & v \\
r & s
\end{array}\right|
$$

in seguito ad un breve calcolo si ba:

$$
\left.\begin{array}{l}
\Delta^{2} \alpha_{1} \equiv(\alpha u+\gamma v) s-(\beta u+\delta v) r \\
\Delta^{2} \beta_{1} \equiv(\beta u+\delta v) u-(\alpha u+\gamma v) v \\
\Delta^{2} \gamma_{1} \equiv(\alpha r+\gamma s) s-(\beta r+\delta s) r \\
\Delta^{2} \delta_{1} \equiv(\beta r+\delta s) u-(\alpha r+\gamma s) v .
\end{array}\right\} \quad(\bmod \eta)
$$

Dalle formole (8) risulta:

$$
\Delta\left(\alpha_{1}+\delta_{1}\right) \equiv \alpha+\delta, \quad \Delta^{2}\left(\alpha_{1} \delta_{1}-\beta_{1} \gamma_{1}\right) \equiv \alpha \delta-\beta \gamma, \quad(\bmod p) .
$$

Io introduco il numero:

$$
D=(\alpha+\delta)^{2}-4(\alpha \delta-\beta \gamma),
$$

e distinguo i tre casi possibili :

$$
\left(\frac{D}{p}\right)=+1,\left(\frac{D}{p}\right)=-1,\left(\frac{D}{p}\right)=0 ;
$$

dove, secondo Legendre, il simbolo $\left(\frac{D}{p}\right)$ denota il resto di $D^{\frac{p-1}{2}}$, che è preso rispetto al numero primo $p$ ed ha il minimo valore assoluto. 
27. Caso $\left(\frac{D}{p}\right)=+1$.

Il minimo intero $q$, positivo o nullo, che verifica la congruenza:

$$
D q-(\alpha+\delta)^{2} \equiv 0,(\bmod p),
$$

in virtù delle $(9)$ resta inalterato per tutte le possibili operazioni :

$$
\left|\begin{array}{ll}
u & v \\
r & s
\end{array}\right|
$$

e quindi è un numero invariantivo per il gruppo rappresentato dal $G_{5}^{2,2}$ definito dalle relazioni (4) e (5) del presente paragrafo.

Ciò posto, il discriminante $D$ della congruenza quadratica :

$$
\rho^{2}-(\alpha+\delta) \rho+(\alpha j-\beta \gamma) \equiv 0, \quad(\bmod p),
$$

$\grave{e}$, nel caso attuale, un numero quadrato rispetto a mod $p$, e perciò la detta congruenza è soddisfatta da due interi $\rho_{1}, \rho_{2}$, che sono distinti rispetto a questo modulo.

E possibile dunque determinare quattro numeri $u, v, r, s$ che verifichino le congruenze:

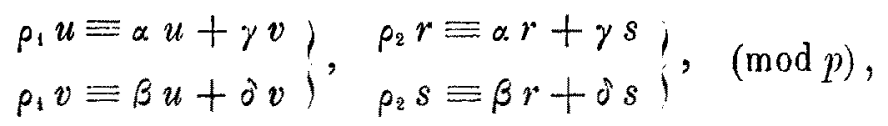

e tali che risulti il determinante $\Delta=u s-v r$ primo con $p$; allora, facendo la trasformazione:

$$
\left|\begin{array}{ll}
u & v \\
v & s
\end{array}\right|
$$

le (8) dànno:

$$
\Delta \alpha_{1} \equiv \rho_{1}, \quad \Delta \delta_{1} \equiv \rho_{2}, \quad \beta_{1} \equiv \gamma_{1} \equiv 0, \quad(\bmod p) .
$$

In conclusione, per i gruppi considerati nel presente caso, le relazioni (5) possono essere sostituite dalle relazioni:

$$
E_{\mathrm{5}}^{p}=E_{2}^{\alpha}, \quad E_{1}^{p}=E_{1}^{\delta}, \quad E_{3}^{p}=1, \quad E_{2}^{p}=1, \quad E_{1}^{p}=1,
$$

dove la differenza $\delta-\alpha$ non è divisibile per $p$.

Prendendo come formole iniziali le (11), mediante le (8) si vede che, eseguendo la trasformazione:

$$
\mid \begin{array}{ll}
u & 0 \\
0 & s
\end{array}
$$


non viene alterata la loro forma, ma $\mathrm{i}$ numeri $\alpha$ e $\partial$ acquistano uno stesso fattore arbitrario primo con $p$ : si può dunque supporre:

$$
\delta-\alpha \equiv 2, \quad(\bmod p) \text {. }
$$

Ciò posto, se la somma $a+\delta$ non è divisibile per $p$, denotando con $\varepsilon$ una radice primitiva di $p$, si può porre:

$$
\alpha \equiv \varepsilon^{m}-1, \quad \delta \equiv \varepsilon^{m}+1, \quad(\bmod p) ;
$$

allora, sostituendo questi valori di $\alpha$ e $\delta$ nella (10), si ottiene:

$$
q \equiv \varepsilon^{2 m}, \quad(\bmod p) \text {. }
$$

Quando si cambia $m$ in $m+\frac{p-1}{2}$, il numero $q$ resta inalterato, ma $\sigma$ e $\delta$ si cambiano rispettivamente in $-\delta$ e $-\alpha$.

Giacchè la trasformazione :

$$
\left|\begin{array}{ll}
0 & 1 \\
1 & 0
\end{array}\right|
$$

produce lo stesso cambiamento, io debbo fare sul numero $m$ solamente le ipotesi :

$$
m=0,1, \ldots, \frac{p-3}{2}
$$

In corrispondenza a questi tali valori di $m$, si hanno $\frac{p-1}{2}$ gruppi $G_{5}{ }^{2,2}$ definiti dalle formole:

$$
E_{5}^{p}=E_{2}^{\varepsilon^{m}-1}, \quad E_{4}^{p}=E_{1}^{e^{m}+1}, \quad E_{3}^{p}=1, \quad E_{2}^{p}=1, \quad E_{1}^{p}=1
$$

e dalle formole (4).

Questi gruppi sono effettivamente distinti, perchè in virtù della (12) due qualunque di essi hanno $\mathrm{i}$ numeri invariantivi $q$ diseguali.

Se la somma $\alpha+\delta$ è divisibile per $p$, il che equivale a supporre $q=0$, si ha:

$$
\alpha \equiv-1, \quad \delta \equiv 1, \quad(\bmod p),
$$

e quindi si ottiene un nuovo gruppo $G_{5}{ }^{2,2}$ corrispondente alle formole:

$$
E_{\mathrm{s}}^{p}=E_{2}^{-1}, \quad E_{4}^{p}=E_{1}, \quad E_{3}^{p}=1, \quad E_{2}^{p}=1, \quad E_{1}^{p}=1
$$


28. Caso $\left(\frac{D}{p}\right)=-1$.

Il numero $q$ che soddisfa alla (10) $\grave{e}$, anche in questo caso, invariantivo per il gruppo rappresentato dal $G_{5}^{2,2}$ definito dalle formole (4) e (5); però, se detto numero non è zero, esso è un non residuo quadratico di $p$.

Giacchè nelle attuali ipotesi, nessuno dei numeri $\beta, \gamma$ può essere multiplo di $p$, è possibile determinare un intero $v$ in modo che sia:

$$
2 \gamma v \equiv \delta-\alpha, \quad(\bmod p) ;
$$

allora, eseguendo la trasformazione :

$$
\left|\begin{array}{ll}
1 & v \\
0 & 1
\end{array}\right|
$$

le (8) dànno $\alpha_{1} \equiv \grave{j}_{1}(\bmod p)$.

Io suppongo dunque che $\mathrm{i}$ valori iniziali di $\alpha$ e $\delta$, presi rapporto a $\bmod p$, abbiano uno stesso valore $\rho$ e cerco tutte le trasformazioni:

$$
\left|\begin{array}{ll}
u & v \\
v & s
\end{array}\right|
$$

che lasciano sussistere questa ipotesi.

Ora, essendo $\alpha \equiv \delta \equiv \rho$, affinchè sia $\alpha_{1} \equiv \delta_{1} \equiv \rho_{1}$, è necessario e sufficiente che $u, v, r, s$ verifichino la congruenza:

$$
\beta u v-\gamma v s \equiv 0, \quad(\bmod p)
$$

e cio risulta subito dalle formole (8).

Supponendo soddisfatta la (13), le (8) si possono sostituire con le formole:

$$
\Delta \rho_{1} \equiv \rho, \quad \Delta^{2} \beta_{1} \equiv \beta u^{2}-\gamma v^{2}, \quad \Delta^{2} \gamma_{1} \equiv \gamma s^{2}-\beta r^{2}, \quad(\bmod p),
$$

mediante le quali si verifica subito che è :

$$
\Delta^{2} \beta_{1} \gamma_{1} \equiv \beta \gamma, \quad(\bmod p) .
$$

Giacche si ha ora $D=4 \beta \gamma$, i due numeri $\beta$ e $\gamma$ sono necessariamente uno residuo e l'altro non residuo quadratico rispetto a $\bmod p$, ed è indifferente attribuire una di queste proprietà all' uno od all'altro numero, perchè si pud dimostrare che esiste una trasformazione la quale, senza alterare $p$, scambia $\beta$ con $\gamma$.

Infatti, se -1 è un numero quadrato $\bmod p$, il che ha luogo soltanto quando $p-1$ è multiplo di 4 , trovato un intero $v$ tale che $v^{2} \equiv-1(\bmod p)$, 
ottengo l'intento mediante la trasformazione:

$$
\mid \begin{array}{ll}
0 & v \\
v & 0
\end{array}
$$

Se invece $p-1$ non è divisibile per 4 , si considerino i $\frac{p+1}{2}$ numeri che si ottengono da $1+v^{2}$ attribuendo a $v$ i valori:

$$
0,1, \ldots, \frac{p-1}{2} \text {. }
$$

I detti numeri risultano distinti rispetto a $\bmod p$, e perciò non possono essere tutti residui quadratici di $p$. Dunque, giacchè per ipotesi non è mai $1+v^{2}$ un multiplo di $p$, nella serie dei valori attribuiti a $v$ ne esiste almeno uno per cui $1+v^{2}$ è un non quadrato $(\bmod p)$.

Allora, fissato per $v$ un tale valore, scelgo, il che riesce possibile, per u ed $s$ una soluzione del sistema:

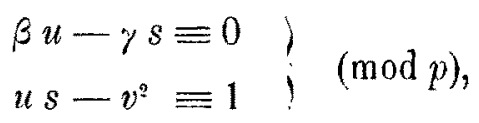

e considero poi la trasformazione:

$$
\begin{array}{ll:l}
u & v & \\
v & s &
\end{array}
$$

Questa trasformazione appartiene alla classe definita dalla formola (13) ed ha il determinante eguale ad $1(\bmod p) ;$ inoltre, le $(14)$ mostrano che essa scambia semplicemente $i$ numeri $\beta$ e $\gamma$.

Sia dunque $\beta$ un residuo quadratico di $p$. Se si pone $v \equiv r \equiv 0(\bmod p)$, la (13) è verificata e le (14) dànno:

$$
u s \rho_{1} \equiv \rho, \quad s^{2} \beta_{1} \equiv \beta, \quad(\bmod p)
$$

perciò, se non è $\rho$ multiplo di $p$, si possono determinare $s$ ed $u$ in modo che sia :

poi la (10) dà :

$$
p_{1} \equiv 1, \quad \beta_{1} \equiv 1, \quad(\bmod p)
$$

$$
y_{1} q \equiv 1, \quad(\bmod p)
$$


Quindi, ponendo $\gamma_{1} \equiv \varepsilon^{2 m+1}(\bmod p)$, si ha:

$$
q \equiv \varepsilon^{-2 m-1}, \quad(\bmod p) \text {. }
$$

In conclusione, ottengo $\frac{p-1}{2}$ gruppi $G_{5}^{2,2}$ che sono definiti dalle (4) e dalle formole che si ottengono da:

$$
E_{5}^{p}=E_{2} E_{1}, \quad E_{4}^{p}=E_{2} E_{1}^{\varepsilon_{1}^{2 n+1}}, \quad E_{3}^{p}=1, \quad E_{2}^{p}=1, \quad E_{1}^{p}=1
$$

facendo successivamente :

$$
m=0,1, \ldots, \frac{p-3}{2}
$$

Questi gruppi sono effettivamente distinti perchè, in virtù della (15), due qualunque di essi hanno $i$ numeri invariantivi $q$ diseguali.

Se invece il numero $\beta$ è un multiplo di $p$, il che equivale a supporre $q=0$, ponendo come prima $v \equiv r \equiv 0(\bmod p)$, le $(14)$ dànno:

$$
s^{2} \beta_{1} \equiv \beta, \quad u^{2} \gamma_{1} \equiv \gamma, \quad(\bmod p) \text {; }
$$

e perciò si possono determinare $s$ ed $u$ in modo che sia:

$$
\beta_{1} \equiv 1, \quad \gamma_{1} \equiv \varepsilon, \quad(\bmod p):
$$

ottengo quindi un nuovo gruppo $G_{z}^{z, 2}$ definito dalle formole:

$$
E_{s}^{p}=E_{1}, \quad E_{4}^{n}=E_{0}^{*}, \quad E_{3}^{p}=1, \quad E_{2}^{p}=1, \quad E_{1}^{n}=1
$$

e dalle relazioni (4).

29. $\operatorname{Caso}\left(\frac{D}{p}\right)=0$.

Si ha allora $D \equiv 0(\bmod p)$ e quindi la congruenza:

$$
p^{2}-(\alpha+\delta) p+(\alpha . j-\beta y) \equiv 0 \quad(\bmod p)
$$

è soddisfatta da un solo resto $\rho$ di $p$.

Scegliendo come numeri $u$ e $v$ una soluzione propria del sistema:

$$
\begin{aligned}
& \rho u \equiv \alpha+\gamma v ; \quad(\bmod p) \\
& \rho v \equiv \beta u+\delta v ;
\end{aligned}
$$


la seconda delle $(8)$ dà $\beta_{1} \equiv 0(\bmod p)$; quindi direttamente dalle (9) si ricava $\Delta \alpha_{1} \equiv \Delta \delta_{1} \equiv p(\bmod p)$.

Assumendo dunque:

le. (8) si scrivono:

$$
\beta \equiv 0, \quad \alpha \equiv \delta \equiv \beta, \quad(\bmod p)
$$

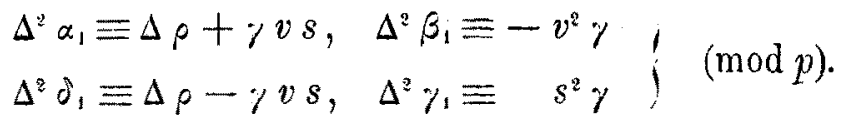

Ciò posto, se y è primo con $p$, volendo conservare le condizioni iniziali, bisogna supporre $v$ nullo $(\bmod p)$; indi si può determinare $u$ in modo che sia $\eta_{1} \equiv 1(\bmod p)$ ovvero $\gamma_{1} \equiv \varepsilon(\bmod p)$. Allora, se p è un multiplo di $p$, si ha $\alpha_{1} \equiv \partial_{1} \equiv 0(\bmod p)$, in caso contrario si possono scegliere $r$ ed $s$ in modo che $\operatorname{sia} \alpha_{1} \equiv \delta_{1} \equiv 1(\bmod p)$.

Se poi è $\gamma$ un multiplo di $p$, si ha sempre $\beta_{1} \equiv \gamma_{1} \equiv 0(\bmod p)$, ma è $\alpha_{1} \equiv \partial_{1} \equiv 0(\bmod p)$, ovvero si può supporre $\alpha_{1} \equiv \delta_{1} \equiv 1(\bmod p)$, secondo che $\grave{e}$, ovvero non è, $\rho$ un multiplo di $p$.

In conclusione, in corrispondenza all' ipotesi $\left(\frac{D}{p}\right)=0$, si hanno soltanto sei gruppi, i quali sono definiti dalle (4) e dalle seguenti modificazioni delle formole $(5)$ :

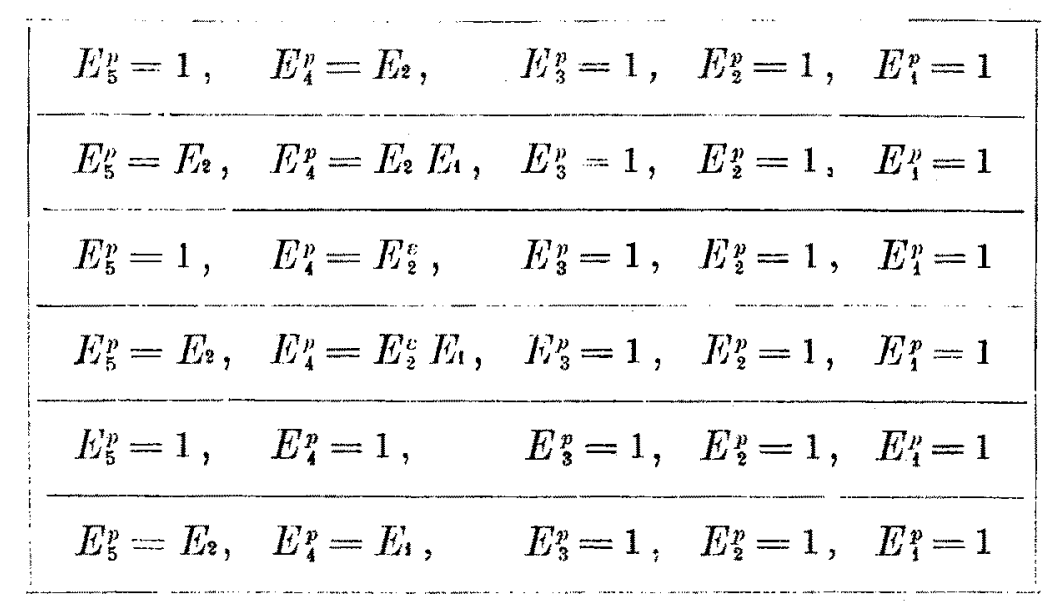

30. Finalmente jo passo ad esaminare il caso di $p=3$, che ho escluso verso la fine del n. $^{\circ} 26$. Quando è $p=3$ tutte le possibili operazioni:

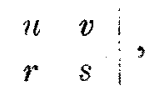


definite precedentemente costituiscono un gruppo di grado 48; però, osservando che l'operazione:

$$
\left|\begin{array}{rr}
-1 & 0 \\
0 & -1
\end{array}\right|
$$

lascia inalterati i quattro numeri $\alpha, \beta, \gamma, \delta$ che figurano nelle (5), io non considero come distinte le due operazioni:

$$
\left|\begin{array}{ll}
u & v \\
r & s
\end{array}\right|, \quad\left|\begin{array}{cc}
-u & -v \\
-v & -s
\end{array}\right|,
$$

ed allora il grado del detto gruppo si riauce di metà. Il gruppo di grado 24, che cosi si ottiene, è isomorfo al gruppo dell'ottaedro $\left({ }^{*}\right)$ : esso può essere generato mediante le tre operazioni:

$$
U=\left|\begin{array}{rr}
-1 & 0 \\
0 & 1
\end{array}, \quad V=\begin{array}{ll}
1 & 1 \\
0 & 1
\end{array}, \quad W=\begin{array}{rr}
1 & 1 \\
-1 & 1
\end{array}\right|,
$$

che sono ordinatamente di secondo, terzo e quarto ordine e che soddisfano alle relazioni :

$$
V^{2} W=W^{3} V, \quad W^{3} U=U W, \quad U V^{2}=V U .
$$

Si vode subito, tenendo presenti le formole (4) e (5), che la $U$ non altera $\beta$ e $\gamma$, ma cambia soltanto di segno a e $\delta$.

Facendo uso della (7), si verifica facilmente che l'operazione $V$ cambia la somma $\alpha+\delta$ in $\alpha+\delta+1$ e quindi l'operazione $V^{2}$ cambia la detta somma in $\alpha+\partial+2$; perciò non si lede la generalità ponendo:

$$
x+i \equiv 0, \quad(\bmod 3) \text {. }
$$

Ciò posto, servendosi della (7) e tenendo conto della congruenza (16), un breve calcolo mostra che l'operazione $W$ produce sopra i numeri $\alpha, \beta, \gamma, \delta$ la sostituzione congrua:

$$
\begin{array}{ll}
\alpha_{1} \equiv \beta+\gamma, & \beta_{1} \equiv \alpha+\beta-\gamma+1, \\
\delta_{1} \equiv-\beta-\gamma, & \gamma_{1} \equiv-\beta+\gamma-\delta-1,
\end{array} \quad(\bmod 3) .
$$

(*) Per convincersene, basta far corrispondere alle tre operazioni che sono donotate in seguito con $\zeta, I, W$, ordinatamente le tre sostituzioni: $(02),(012),(0) \perp 23)$. 
il cui grado è la quinta potenza di un numero primo.

Dunque le operazioni $W$ ed $U$ lasciano inalterata la (16) e lo stesso fa ogni operazione del gruppo di grado $\&$ da esse generato; mentre una qualunque delle rimanenti 16 operazioni, potendosi ottenere come prodotto di una operazione del detto gruppo di grado 8 per $V$, ovvero per $V^{2}$, non laseia sussistere la congruenza (16). In virtù di questa congruenza, relativamente ai valori di $\alpha$ e $j$, si possono fare le seguenti tre ipotesi :

$$
\alpha \equiv 0, \delta \equiv 0 ; \quad \alpha \equiv 1, \delta \equiv 2 ; \quad \alpha \equiv 2, \delta \equiv 1, \quad(\bmod 3) ;
$$

poi a ciascuna di queste tre coppie si può associare una coppia qualunque di valori di $\beta$ e $\gamma$ : si ottengono così 27 quaterne di numeri $(\alpha \beta \gamma \delta)$ a ciascuna delle quali corrisponde un gruppo $G_{5}^{2,2}$ di grado $3^{5}=243$.

Pero due quaterne, che si deducono l'una dall'altra mediante una operazione del precedente gruppo di grado 8 , si debbono ritenere equivalenti in quanto che esse definiscono due gruppi jsomorfi di grado 243.

Nella tabella che segue io scrivo tutte le 27 quaterne, mettendo in una stessa linea orizzontale le quaterne che sono equivalenti.

\begin{tabular}{|c|c|c|c|c|c|c|c|}
\hline$\left(\begin{array}{llll}1 & 0 & 1 & 2\end{array}\right)$ & $\left(\begin{array}{llll}1 & 1 & 1 & 2\end{array}\right)$ & $\left(\begin{array}{llll}2 & 2 & 0 & 1\end{array}\right)$ & $(2221)$ & $\left(\begin{array}{llll}2 & 0 & 1 & 1\end{array}\right)$ & $\left(\begin{array}{lllll}2 & 1 & 1 & 1\end{array}\right)$ & $\left(\begin{array}{llll}1 & 2 & 0 & 2\end{array}\right)$ & $\left(\begin{array}{lll}1222\end{array}\right)$ \\
\hline$(1022)$ & $\left(\begin{array}{llll}2 & 0 & 2 & 1\end{array}\right)$ & $\left(\begin{array}{llll}2 & 1 & 0 & 1\end{array}\right)$ & $\left(\begin{array}{llll}1 & 1 & 0 & 2\end{array}\right)$ & & & & \\
\hline$\left(\begin{array}{llll}0 & 1 & 1 & 0\end{array}\right)$ & $\left(\begin{array}{llll}2 & 1 & 2 & 1\end{array}\right)$ & $\left(\begin{array}{llll}0 & 2 & 2 & 0\end{array}\right)$ & $\left(\begin{array}{llll}1 & 1 & 2 & 2\end{array}\right)$ & & & & \\
\hline$\left(\begin{array}{llll}0 & 0 & 1 & 0\end{array}\right)$ & $(1002)$ & $\left(\begin{array}{llll}0 & 2 & 0 & 0\end{array}\right)$ & $\left(\begin{array}{llll}2 & 0 & 0 & 1\end{array}\right)$ & & & & \\
\hline$(0020)$ & $\left(\begin{array}{llll}2 & 2 & 1 & 1\end{array}\right)$ & $\left(\begin{array}{llll}0 & 1 & 0 & 0\end{array}\right)$ & $\left(\begin{array}{llll}1 & 2 & 1 & 2\end{array}\right)$ & & & & \\
\hline$\left(\begin{array}{llll}0 & 0 & 0 & 0\end{array}\right)$ & & & & & & & \\
\hline$(0210)$ & & & & & & & \\
\hline
\end{tabular}

Si vede dunque che esistono soltanto sette gruppi $G_{3}^{2,2}$ di grado 243 , che non posseggono alcun divisore Abeliano d'indice 3 e questi gruppi si posson fare corrispondere alle sette quaterne $(\alpha \beta \gamma \delta)$, che stanno nella prima linea verticale della precedente tabella. 
Io scrivo le formole che, insieme alle (4), servono a definire i detti gruppi.

\begin{tabular}{|c|c|c|}
\hline$E_{5}^{3}=E_{2}$ & $E_{4}^{3}=E_{2} E_{1}^{2}$ & $E_{3}^{3}=1, \quad E_{2}^{3}=1, \quad E_{1}^{3}=1$ \\
\hline$E_{5}^{3}=E_{2}$ & $E_{4}^{3}=E_{2}^{2} E_{1}^{2}$ & $E_{3}^{3}=1, \quad E_{2}^{3}=1, \quad E_{1}^{3}=1$ \\
\hline$E_{\mathrm{s}}^{3}=E_{\mathrm{t}}$ & $E_{4}^{3}=E_{2}$ & $E_{3}^{3}=1, \quad E_{2}^{3}=1, \quad E_{1}^{3}=1$ \\
\hline$E_{5}^{3}=1$ & $E_{4}^{3}=E_{2}$ & $E_{3}^{3}=1, \quad E_{2}^{3}=1, \quad E_{1}^{3}=1$ \\
\hline$E_{5}^{3}=1$ & $E_{4}^{2}=E_{2}^{2}$ & $E_{3}^{3}=1, \quad E_{2}^{3}=1, \quad E_{1}^{3}=1$ \\
\hline$E_{5}^{3}=1$ & $E_{4}^{3}=1$ & $E_{3}^{3}=1, \quad E_{2}^{3}=1, \quad E_{1}^{3}=1$ \\
\hline$E_{5}^{3}=E_{1}^{2}$ & $E_{4}^{3}=E_{2}$ & $E_{3}^{3}=1, \quad E_{2}^{3}=1, \quad E_{1}^{3}=1$ \\
\hline
\end{tabular}

31. Nel presente paragrafo io ho stabilito quanto segue.

Ogni gruppo $G_{5}^{2,2}$, che non possiede alcun divisore Abeliano d'indice $r$, è perfettamente definito dalle formole (4) e (5), purchè si tenga presente che $E_{2}$ ed $E_{1}$ appartengono al divisore invertibile $H$.

Non esistono di tali gruppi di grado $2^{5}=32$.

Esistono soltanto sette di tali gruppi di grado $3^{5}=243$.

Finalmente, quando il numero primo $p$ supera 3 , esistono $p+7$, e non più, di tali gruppi, i quali, rispetto alle formole (4) e (5), si classificano nella seguente maniera.

Quando gl'interi $\alpha, \beta, \gamma, \delta$ verificano la congruenza:

$$
D=(\alpha+j)^{2}-4(\alpha \partial-\beta \gamma) \equiv 0,(\bmod p),
$$

si ha uno dei sei gruppi trovati al n. ${ }^{\circ} 29$; in ogni altro caso, il minimo numero $q$ positivo o nullo, che verifica la congruenza:

$$
D q-(\alpha+\delta)^{2} \equiv 0,(\bmod p)
$$

è un numero invariantivo per il gruppo definito dalla quaterna $(\alpha \beta \% j)$.

In corrispondenza ad ogni numero $q>0$ si ha un solo gruppo; ma, per $q=0$, si hanno due gruppi secondo che $D \grave{e}$, ovvero non $\grave{e}$, un residuo quadratico di $p$.

I diversi tipi di gruppi trovati nel presente paragrafo sono rappresentati nella seguente tabella. 
il cui grado è la quinta potenza di un numero primo.

I.

$\left\{H\left(E_{2}, E_{1}\right)\right\},\left\{K\left(E_{3}, E_{2}, E_{1}\right)\right\}$.

$E_{5}^{-1} E_{4} E_{5}=E_{4} E_{3}, \quad E_{5}^{-1} E_{3} E_{5}=E_{3} E_{2}, \quad E_{4}^{-1} E_{3} E_{4}=E_{3} E_{1}$.

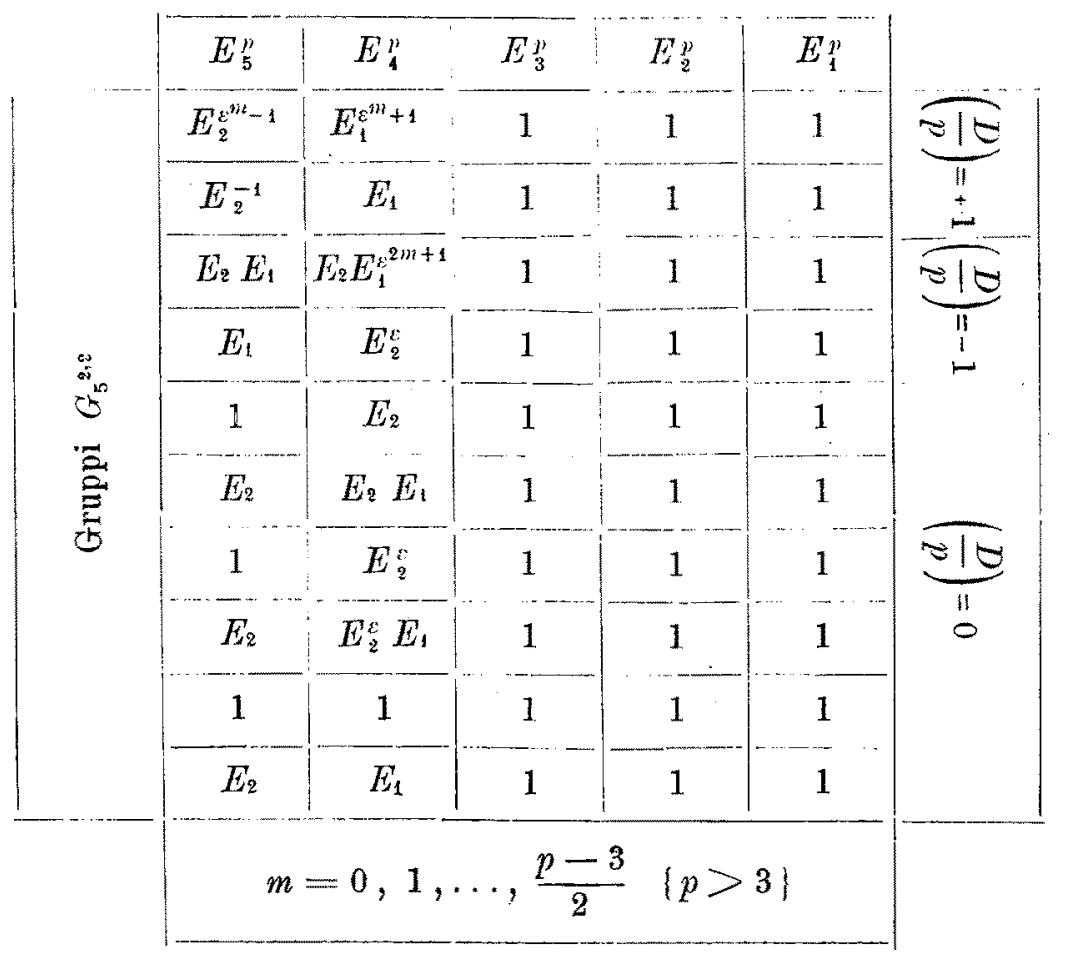

II.

\begin{tabular}{|c|c|c|c|c|c|}
\hline & $E_{5}^{3}$ & $E_{4}^{3}$ & $E_{3}^{3}$ & $E_{2}^{3}$ & $E_{1}^{3}$ \\
\hline \multirow{3}{*}{ 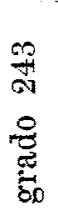 } & $E_{2}$ & $E_{2} E_{1}^{2}$ & 1 & 1 & 1 \\
\hline & $E_{z}$ & $E_{2}^{2} E_{1}^{2}$ & 1 & 1 & 1 \\
\hline & $E_{\mathrm{i}}$ & $E_{2}$ & 1 & 1 & 1 \\
\hline \multirow{4}{*}{ 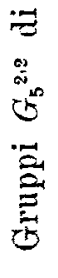 } & 1 & $E_{2}$ & 1 & 1 & 1 \\
\hline & 1 & $E_{2}^{2}$ & 1 & 1 & 1 \\
\hline & 1 & 1 & 1 & 1 & 1 \\
\hline & $E_{1}^{2}$ & $E_{2}$ & 1 & 1 & 1 \\
\hline
\end{tabular}




\section{$\S$ V. I Gruppi $G_{5}{ }^{2}$ che posseggono un divisore Abeliano d'indice $p$.}

32. Al principio del n. $^{0} 25$ si è osservato che un gruppo $G_{5}{ }^{2}$ può contenere uno oppure nessuno divisore Abeliano d'indice $p$. Io ho svolto nel paragrafo precedente l'analisi relativa alla seconda ipotesi ed ora mi propongo di svolgere in questo paragrafo l'analisi relativa alla prima.

Il divisore Abeliano $G_{4}{ }^{0}$ contenuto per ipotesi in $G_{5}{ }^{2}$, contiene evidentemente il divisore invertibile $H$, e quindi è possibile costruire una serie canonica di composizione:

tale che sia $G_{2}=H$.

$$
G_{5}{ }^{2}, G_{4}^{0}, G_{3}, G_{2}, G_{1},
$$

Denotando con:

$$
E_{5}, \quad E_{4}, E_{3}, E_{2}, E_{1}
$$

una base relativa alla detta serie e scrivendo le formole:

$$
E_{5}^{-1} E_{4} E_{5}=E_{4} E_{3}^{\prime}, \quad E_{5}^{-1} E_{3} E_{5}=E_{3} E_{2}^{\prime}, \quad E_{4}^{-1} E_{3} E_{4}=E_{3},
$$

l'elemento $E^{\prime \prime}$; può essere contenuto o non essere contenuto in $H$. Questi due casi si debbono distinguere accuratamente, perchè, in corrispondenza, si hanno due classi di gruppi $G_{5}{ }^{2}$ ben diverse.

Comincio ad occuparmi del primo caso, il quale è equivalente all'ipotesi di ritenere il gruppo $K$ coincidente col gruppo $H$, perchè, in virtù del ragionamento fatto al $n .^{\circ} 24$, in tal caso, anche quando è $p=2$, la potenza $p^{\text {mat }}$ di un elemento qualunque di $G_{\mathrm{s}}{ }^{2}$ sta in $H$.

Dunque deve essere $E_{3}^{\prime \prime}=1, E_{2}^{\prime p}=1$; e siccome gli elementi $E_{2}^{\prime}, E_{2}^{\prime}$ non possono appartenere ad uno stesso gruppo ciclico (n. $\left.{ }^{\circ} 24\right)$, i detti elementi sono di grado $p$ e possono generare il gruppo $H$.

Io posso dunque scrivere le formole:

$$
\begin{gathered}
E_{5}^{-1} E_{1} E_{5}=E_{1} E_{2}, E_{5}^{1} E_{3} E_{5}^{\prime}=E_{3} E_{1}, E_{1}^{-1} E_{3} E_{1}=E_{3}, \\
E_{5}^{p}=E_{2}^{\lambda} E_{1}^{\prime \prime}, E_{1}^{\prime \prime}=E_{2}^{\prime \prime} E_{1}^{\prime \prime}, E_{3}^{p}=E_{2}^{\prime} E_{1}^{\lambda}, E_{2}^{p}=1, E_{1}^{\prime \prime}=1 .
\end{gathered}
$$

Queste formole definiscono, qualunque siano gl'interi $\lambda, \mu, \alpha, \beta, \%, \delta$, un gruppo $G_{5}^{2}$, e tutti i gruppi così definiti appartengono evidentemente alla classe dei gruppi $G_{\mathrm{s}}{ }^{2,3}$.

Si consideri una nuova base canonica:

$$
\mathbf{F}_{5}, \mathbf{E}_{4}, \mathbf{E}_{3}, \mathbf{E}_{2}, \mathbf{E}_{1},
$$


tale che l'operazione $T$ che porta questa nuova hase nella primitiva non alteri le prime tre delle relazioni (1).

Ponendo:

$$
\mathbf{E}_{\mathbf{8}} \equiv E_{5}, \quad \mathbf{E}_{4} \equiv E_{4}^{r} E_{3}^{r}, \quad \mathbf{E}_{3} \equiv E_{4}^{r} E_{3}^{s}, \quad\left(\bmod G_{2}\right),
$$

si trova:

$$
\mathbf{E}_{2} \equiv E_{2}^{u} E_{1}^{r}, \quad \mathbf{E}_{1} \equiv E_{2}^{r} E_{1}^{s} ;
$$

e giacchè $\mathbf{E}_{2}$ ed $\mathbf{E}_{1}$ sono due elementi generatori di $H$, deve supporsi il determinante $\Delta=u s-v r$ primo con $p$. Per la osservazione più volte fatta, considero come una sola operazione tutte le operazioni $T$ appartenenti alla classe definita dalle (2), quando si attribuiscono ad $u, v, r, s$ determinati resti di $p$, e quest' unica operazione la rappresento col simbolo:

$$
\left|\begin{array}{ll}
u & v \\
r & s
\end{array}\right|
$$

Inoltre, se si cambia soltanto l'elemento $\mathbf{E}_{\mathbf{s}}$ ponendo, nella maniera pù generale,

$$
\mathbf{E}_{5} \equiv E_{5}^{4}, \quad\left(\bmod G_{4}{ }^{0}\right),
$$

essendo $\sigma$ primo con $p$, risulta subito dalle (1) che $\mathrm{i}$ quattro numeri $\alpha, \beta, \gamma, \delta$ acquistano uno stesso fattore arbitrario primo con $p$ : dunque le due quaterne $(\alpha, \beta, \gamma, \delta),(\sigma \alpha, \sigma \beta, \sigma \gamma, \sigma \delta)$ debbono ritenersi equivalenti.

Ciò posto, eseguendo l'operazione :

$$
\left|\begin{array}{ll}
u & v \\
r & s
\end{array}\right|
$$

si trovano facilmente le relazioni:

$$
\left.\begin{array}{l}
\Delta \alpha_{1} \equiv(\alpha u+\gamma v) s-(\beta u+\delta v) v \\
\Delta \beta_{1} \equiv(\beta u+\delta v) u-(\alpha u+\gamma v) v \\
\Delta \gamma_{1} \equiv(\alpha r+\gamma s) s-(\beta r+\delta s) r \\
\Delta \delta_{1} \equiv(\beta r+\delta s) u-(\alpha r+\gamma s) v
\end{array}\right\} \quad(\bmod p)
$$

dalle quali risulta:

$$
\alpha_{1}+\delta_{1} \equiv \alpha+\delta, \quad \alpha_{1} \delta_{1}-\beta_{1} \gamma_{1} \equiv \alpha \delta-\beta \%, \quad(\bmod p) .
$$

Allora, supponendo $p>2$, introduco il numero:

$$
D=(\alpha+\partial)^{2}-4(\alpha \delta-\beta \gamma),
$$


e distinguo, come nel paragrafo precedente, $\mathrm{i}$ tre casi di:

$$
\left(\frac{D}{p}\right)=+1, \quad\left(\frac{D}{p}\right)=-1, \quad\left(\frac{D}{p}\right)=0 .
$$

33. Nei primi due casi, il minimo numero intero $q$, positivo o nullo, che verifica la congruenza:

$$
D q-(\alpha+\delta)^{2} \equiv 0, \quad(\bmod p),
$$

resta inalterato per tutte le possibili operazioni $T$, perchè, quando si moltiplicano i quattro numeri $\alpha, \beta, \gamma, \delta$ per uno stesso fattore $\sigma$ primo con $p$, i numeri $D$ ed $(\alpha+\partial)^{2}$ acquistano lo stesso fattore $\sigma^{2}$; quindi $q$ ha carattere invariantivo per il gruppo rappresentato dal $G_{5}^{2,3}$ definito dalle relazioni (1) del presente paragrafo.

$$
\begin{aligned}
& \text { Quando ̀̀ }\left(\frac{D}{p}\right)=+1 \text {, la congruenza: } \\
& \qquad \rho^{2}-(\alpha+\delta) \rho+(\alpha \delta-\beta y) \equiv 0,(\bmod p),
\end{aligned}
$$

è soddisfatta da due distinti resti di $p$; mentre quando è $\left(\frac{D}{p}\right)=-1$, non esiste alcun numero intero che verifichi la detta congruenza; allora, relativamente alla quaterna di numeri $\alpha, \beta, \gamma, \delta$, l'analisi procede in modo perfettamente analogo a quella dei n. 27 e 28 del $\S$ IV e non è necessario ripeterla qui.

In riguardo ai numeri $\lambda$ e $\mu$. io faccio la seguente osservazione.

Cambiando solamente l'elemento $\mathbf{E}_{5}$ che figura nelle (2), ponendo:

$$
\mathbf{E}_{5} \equiv E_{5} E_{4}^{h} E_{3}^{k}, \quad\left(\bmod G_{2}\right),
$$

i numeri $\alpha, \beta, \gamma, \partial$ restano inalterati, ma $\lambda$ e $\mu$ si cambiano rispettivamente in due numeri $\lambda_{1}$ e $\mu_{1}$ tali che:

$$
\begin{aligned}
& \lambda_{1} \equiv \lambda+\alpha h+\gamma k \\
& \mu_{1} \equiv \mu+\beta h+j k
\end{aligned}, \quad(\bmod p) .
$$

Infatti, dalle formule (1) si ricava:

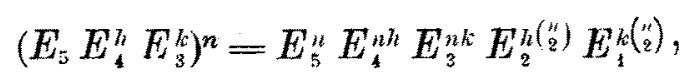

e quindi, avendo supposto $p>2$, si ha:

$$
\mathbf{E}_{5}^{p}=E_{5}^{p} E_{4}^{p h} E_{3}^{p k} \text {. }
$$


Ciò posto, quando il determinante $\alpha \grave{j}-\beta \gamma$ è primo con $p$, cioè quando $E_{4}^{p}$ ed $E_{3}^{p}$ sono due elementi generatori del gruppo $H$, si possono determinare gl' interi $h$ e $k$ in modo che sia :

$$
\lambda_{1} \equiv \mu_{1} \equiv 0, \quad(\bmod p) ;
$$

dunque, in tale ipotesi, è lecito supporre $E_{s}^{p}=1$.

Fatte queste considerazioni, si vede che nel caso di $\left(\frac{D}{p}\right)=+1$, si hanno $\frac{p-1}{2}$ gruppi $G_{5}^{2,3}$ in corrispondenza alle seguenti modificazioni delle ultime einque formole (1):

$$
E_{5}^{p}=1, \quad E_{4}^{p}=E_{2}^{e^{m-1}}, \quad E_{3}^{p}=E_{1}^{c^{m_{1}+1}}, \quad E_{2}^{p}=1, \quad E_{1}^{p}=1
$$

essendo $m=0, \ldots, \frac{p-3}{2}$.

Oltre ai detti $\frac{p-1}{2}$ gruppi, esistono ancora altri due particolari gruppi: il primo di questi si presenta quando il numero invariantivo $q$ è nullo. In tal caso si ha un gruppo che corrisponde alle formole:

$$
E_{5}^{p}=1, \quad E_{1}^{p}=E_{2}^{-1}, \quad E_{3}^{p}=E_{1}, \quad E_{2}^{p}=1, \quad E_{1}^{p}=1
$$

Il secondo gruppo particolare si presenta qualora si osservi che, nell'ipotesi di $m=0$, non è sempre lecito supporre nelle (7) $E_{\mathrm{5}}^{p}=1$, perchè, nella detta ipotesi, $E_{4}^{p}$ ed $E_{3}^{p}$ non sono due elementi generatori di $H$. Ma essendo, nel caso attuale, $\alpha=\beta=\gamma=0$ e $\delta=2$, le (5) mostrano che si può sempre supporre $\mu=0$; poi, se non è $\lambda$ un multiplo di $p$, chiamando $E_{4}$ l'elemento $E_{4}^{\lambda}$, dalle (1) risulta che si può ritenere $\lambda=1$. Ottengo così un nuovo gruppo $G_{5}{ }^{2,3}$ corrispondente alle formole:

$$
E_{5}^{p}=E_{2}, \quad E_{4}^{p}=1, \quad E_{3}^{p}=E_{1}, \quad E_{2}^{p}=1, \quad E_{1}^{p}=1
$$

Nel caso di $\left(\frac{D}{p}\right)=-1$ si hanno $\frac{p-1}{2}$ gruppi $G_{5,2,3}$ sostituendo le ultime cinque delle relazioni (1) con:

$$
E_{5}^{p}=1, \quad E_{4}^{p}=E_{2} E_{1}, \quad E_{3}^{p}=E_{2} E_{1}^{r^{2 n+1}}, \quad E_{2}^{p}=1, \quad E_{1}^{p}=1
$$


e facendo poi successivamente:

$$
m=0, \ldots, \frac{p-3}{2}
$$

inoltre, si ha un nuovo gruppo $G_{5}{ }^{2,3}$ nell' ipotesi di $q=0$ e questo gruppo corrisponde alle formole:

$$
E_{5}^{p}=1, \quad E_{4}^{p}=E_{1}, \quad E_{3}^{p}=E_{2}^{\varepsilon}, \quad E_{2}^{p}=1, \quad E_{1}^{p}=1
$$

34. $\operatorname{Caso}\left(\frac{D}{p}\right)=0$.

Si ha allora $D \equiv 0(\bmod p)$ e quindi la congruenza:

$$
p^{2}-(\alpha+\delta) p+(\alpha \delta-\beta \gamma) \equiv 0 \quad(\bmod p)
$$

è soddisfatta da un solo resto $\rho$ di $p$; poi, ragionando come nel n. ${ }^{\circ} 29$, si vede che è lecito supporre:

$$
\beta \equiv 0, \quad \alpha \equiv \delta \equiv \rho \quad(\bmod p)
$$

e quindi le formole (3) si scrivono:

$$
\left.\begin{array}{l}
\Delta \alpha_{1} \equiv \Delta \rho+\gamma v s, \quad \Delta \beta_{1} \equiv-v^{2} \gamma \\
\Delta \delta_{1} \equiv \Delta \rho-\gamma v s, \quad \Delta \gamma_{1} \equiv s^{2} \gamma
\end{array}\right\}(\bmod p) .
$$

Ciò posto, se $\gamma$ è primo con $p$, volendo conservare le condizioni iniziali, bisogna supporre $v \equiv 0(\bmod p)$; indi, se $\rho$ è primo con $p$, scelgo gl'interi $u$ ed $s$ in modo che sia $\gamma_{1} \equiv \rho(\bmod p)$, e se $\rho$ è un multiplo di $p$, scelgo i detti interi in modo che sia $y_{1}=1(\bmod p)$. Dunque, se è $\gamma$ primo con $p$, giacchè $\alpha, \beta, \gamma, \delta$ si possono moltiplicare per un fattore arbitrario $\left(\mathrm{n}^{\circ} 32\right)$, è lecito assumere :

ovvero:

$$
\alpha=\delta=\gamma=1, \quad \beta=0,
$$

$$
\alpha=\delta=\beta=0, \quad \gamma=1 \text {. }
$$

Se invece $\gamma$ è un multiplo di $p$, si vede subito che si può assumere:

ovvero :

$$
\alpha=\partial=1, \beta=y=0 \text {, }
$$

$$
\alpha=j=0, \beta=\gamma=0 \text {. }
$$

Nel primo e nel terzo di questi quattro casi, per l'osservazione fatta nel n. ${ }^{\circ}$ precedente, suppongo $\lambda=0, \mu=0$; ma il secondo e quarto caso si scindono oguuno in due dietro le considerazioni che seguono. 
Quando $\grave{\mathrm{e}} \alpha=\delta=\beta=0$ e $\gamma=1$, le (5) mostrano che si può supporre $\lambda=0$ : allora, se $\mu$ è un multiplo di $p$, si ha $E_{\mathrm{s}}^{p}=1$; e se $\mu$ è primo con $p$, eseguendo l'operazione:

$$
\left|\begin{array}{ll}
\mu & 0 \\
0 & \mu
\end{array}\right|
$$

$\alpha, \beta, \gamma, \delta$ non si alterano e $\mu$. va nel resto 1 di $p$.

Quando è $\alpha=\beta=y=\delta=0$ ed uno dei due numeri $\lambda, \mu$ è primo con $p$, una delle due operazioni :

$$
\left|\begin{array}{ll}
1 & 0 \\
\lambda & \mu
\end{array}\right|, \quad\left|\begin{array}{ll}
0 & 1 \\
\lambda & \mu
\end{array}\right|
$$

ha il determinante primo con $p$, e questa tale operazione dà $E_{5}^{\prime \prime}=E_{1}$; se poi i numeri $\lambda, \mu$ sono entrambi multipli di $p$, allora è $E_{5}^{p}=1$.

In conclusione, nel caso $\left(\frac{D}{p}\right)=0$, si hanno sei gruppi $G_{5}^{2,3}$ i quali cor-

\begin{tabular}{|c|c|c|c|}
\hline$E_{5}^{p}=1$ & $E_{*}^{p}=E_{2}$ & $E_{3}^{p}=E_{2} E_{1}$ & $E_{2}^{p}=1, \quad E_{1}^{p}=1$ \\
\hline$E_{s}^{p}=1$ & $E_{4}^{p}=E_{2}$ & $E_{3}^{p}=E_{1}$ & $E_{2}^{p}=1, \quad E_{1}^{p}=1$ \\
\hline$E_{5}^{\prime \prime}=1$ & $E_{4}^{p}=1$ & $E_{3}^{p}=E_{2}$ & $E_{2}^{p}=1, \quad E_{1}^{p}=1$ \\
\hline$E_{5}^{p}=E_{1}$ & $E_{4}^{p}=1$ & $E_{3}^{p}=E_{2}$ & $E_{2}^{p}=1, \quad E_{1}^{p}=1$ \\
\hline$E_{5}^{p}=E_{\mathrm{l}}$ & $E_{4}^{p}=1$ & $E_{3}^{n}=1$ & $E_{2}^{p}=1, \quad E_{.1}^{y}=1$ \\
\hline$E_{5}^{p}=1$ & $E_{4}^{p}=1$ & $E_{3}^{p}=1$ & $E_{2}^{p}=1, \quad E_{1}^{p}=1$ \\
\hline
\end{tabular}
rispondono alle seguenti modificazioni delle ultime cinque formole (1):

35. Io prendo ora in esame il caso di $p=2$ che fu escluso alla fine del $n .^{\circ} 32$.

In questo caso, tutte le operazioni :

$$
\left|\begin{array}{ll}
u & v \\
r & s
\end{array}\right|
$$

costituiscono un gruppo di grado 6 , che è isomorfo a quello che si è presentato nel n." 9 del $\$$ II. 
Le due operazioni :

$$
U=\begin{array}{ll}
1 & 1 \\
0 & 1
\end{array}|, \quad V=| \begin{array}{ll}
0 & 1 \\
1 & 1
\end{array} \mid
$$

generatrici del detto gruppo producono sopra i numeri $\alpha, \beta, \gamma, \sigma$ le due sostituzioni congrue:

$$
\begin{array}{lll}
\alpha_{1} \equiv \alpha+\gamma, & \beta_{1} \equiv \alpha+\beta+\gamma+\gamma, \quad \gamma_{1} \equiv \gamma, \quad \delta_{1} \equiv \gamma+\delta ; \\
\alpha_{1} \equiv \gamma+\delta, & \beta_{1} \equiv \gamma, \quad \gamma_{1} \equiv \alpha+\beta+\gamma+\delta, \quad \delta_{1} \equiv \alpha+\gamma ;
\end{array} \quad(\bmod 2),
$$

rispettivamente; e ciò si verifica subito mediante le (3).

Siccome ognuno dei numeri $\alpha, \beta, \%, \delta$ puo ritenersi, indipendentemente, eguale a 0 od eguale ad 1 , si hanno 16 quaterne $(\alpha \beta y \delta)$; però io assumo come equivalenti due tali quaterne che si deducono l'una dall'altra eseguendo una operazione composta con $U$ e $V$.

Nella tabella che segue sono scritte tutte le 16 quaterne in modo che le quaterne equivalenti stanno in una stessa linea orizzontale.

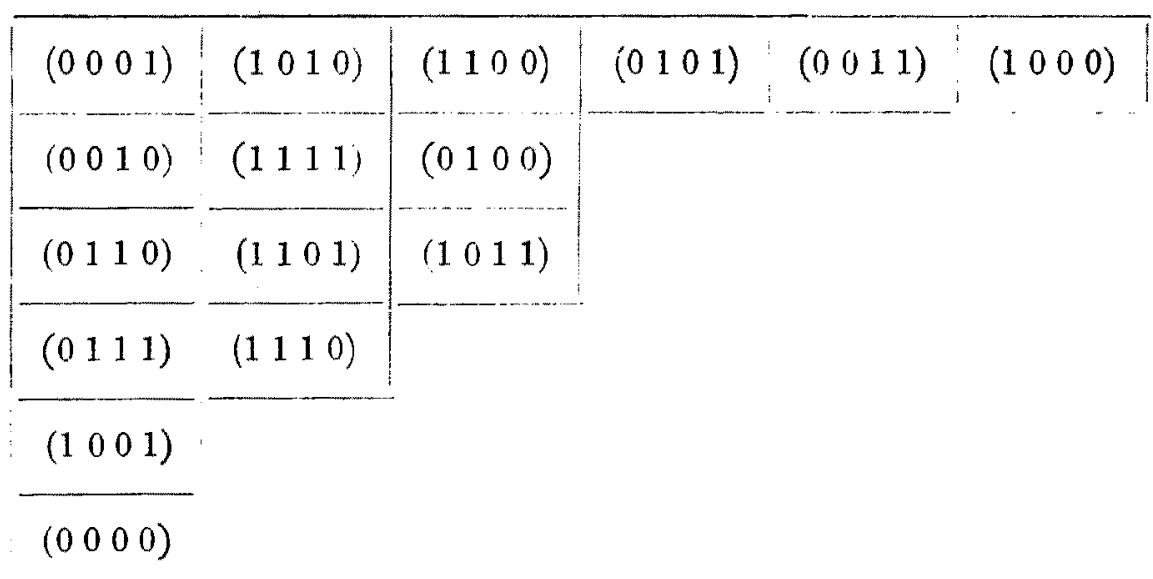

Allora jo considero le sei quaterne che figurano nella prima verticale del precedente quadro e, per ognuna di esse, mi formo il gruppo $a \beta \gamma^{j}$ costituito da tutte operazioni :

$$
\left(\begin{array}{lllll}
E_{5}, & E_{4}^{\prime}, & E_{3}^{\prime}, & E_{2}^{\prime}, & E_{1} \\
\mathbf{F}_{5}, & \mathbf{F}_{4}, & \mathbf{F}_{3}, & \mathbf{F}_{2}, & \mathbf{F}_{1}
\end{array}\right)
$$

che lasciano inalterata la quaterna $(\alpha, \notin ; 0)$. 
Chiamando $W$ una qualunque delle quattro operazioni definite dalle formole:

$$
\mathbf{E}_{5} \equiv E_{5} E_{4}^{h} E_{3}^{k}, \quad \mathbf{E}_{4} \equiv E_{4}, \quad \mathbf{E}_{3} \equiv E_{3}, \quad\left(\bmod G_{2}\right)
$$

è evidente che il gruppo generato dalle operazioni $W$ e dalle operazioni $U, V$ contiene tutte le possibili trasformazioni $T$.

Il detto gruppo è isomorfo al gruppo dell' ottaedro: alle quattro operazioni $W$ corrispondono le quattro sostituzioni del gruppo quaternario normale.

Per rendere chiaro quel che segue, scrivo le relazioni:

$$
\left(E_{5} E_{4}\right)^{2}=E_{5}^{2} E_{4}^{2} E_{2}, \quad\left(E_{5} E_{3}\right)^{2}=E_{5}^{2} E_{3}^{2} E_{1},
$$

che si possono, se più piace, ricavare dalla (6).

Cio posto, il gruppo $\overline{0001}$ è costituito dalle operazioni $W$ : una qualunque di esse o lascia inalterati $i$ numeri $\lambda$ e $\mu$ oppure cambia soltanto $\lambda$ in $\lambda+1$; dunque, associando alla quaterna $\left(\begin{array}{llll}0 & 0 & 0 & 1\end{array}\right)$ una delle due coppie $(00),(01)$ come coppia $(\lambda \mu)$, si ottengono in corrispondenza due grappi $G_{5}^{2,3}$ distinti di grado 32.

Il gruppo $\overline{0010}$ è generato dalle operazioni $W$ e dall'operazione $U V$, e mediante le $W$ si può portare la coppia $(\lambda \mu)$ in $(00)$.

Il gruppo $\overline{0110}$ è generato dalle operazioni $W$ e dalla operazione $U V^{2}$ : una qualunque $W$ o non altera $\mathrm{i}$ numeri $\lambda$ e $\mu$ oppure aumenta i detti numeri contemporaneamente di una unità; e la $U V^{s}$ scambia semplicemente $\lambda$.

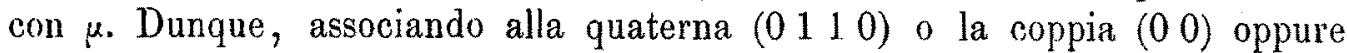
la coppia (0 1) si ottengono, in corrispondenza, due gruppi distinti $G_{5}^{2,3} \mathrm{dj}$ grado 32 .

Il gruppo $\overline{0111}$ è generato dalla operazione $V$ e dalle $W$ e mediante le $W$ si può portare la coppia $(\lambda \mu)$ nella coppia $(00)$.

Il gruppo $\overline{1001}$ è il gruppo principale generato dalle $W$ e dalle $U, V$ : una qualunque delle $W$ non altera $\lambda$ e $\mu$; l'operazione $U$ cambia soltanto $\mu$ in $\mu+\lambda$ e l'operazione $V$ cambia soltanto $\lambda$ in $\lambda+\mu$. Dunque, associando alla quaterna (lllll $\left.\begin{array}{lll}1 & 0 & 1\end{array}\right)$ la coppia ( 000$)$ oppure la coppia $(01)$, si ottengono, in corrispondenza, due gruppi distinti $G_{5}{ }^{2,3}$ di grado 32 .

Finalmente, il gruppo $\overline{0000}$ coincide anche col detto gruppo principale e mediante le $W$ si può portare la coppia $(\lambda \mu)$ nella coppia $(00)$.

La conclusione della precedente analisi è che, nelle attuali ipotesi, esistono nove gruppi $G_{5}^{2,3}$ di grado 32 , e questi gruppi corrispondono alle seguenti modificazioni delle ultime cinque delle formole (1). 


\begin{tabular}{|c|c|c|c|}
\hline$E_{5}^{2}=1$ & $E_{4}^{2}=1$ & $E_{3}^{2}=E_{1}$ & $E_{2}^{2}=1, \quad E_{1}^{2}=1$ \\
\hline$E_{5}^{2}=E_{\mathrm{t}}$ & $E_{4}^{2}=1$ & $E_{3}^{z}=E_{\mathrm{f}}$ & $E_{2}^{2}=1, \quad E_{1}^{2}=1$ \\
\hline$E_{5}^{2}=1$ & $E_{4}^{2}=1$ & $E_{3}^{2}=E_{2}$ & $E_{\mathrm{z}}^{\mathrm{q}}=1, \quad E_{\mathrm{i}}^{\mathrm{q}}=1$ \\
\hline$E_{\mathrm{5}}^{2}=1$ & $E_{4}^{2}=E_{1}$ & $E_{3}^{2}=E_{2}$ & $E_{2}^{2}=1, \quad E_{1}^{2}=1$ \\
\hline$E_{5}^{q}=E_{\mathfrak{t}}$ & $E_{4}^{2}=E_{1}$ & $E_{3}^{2}=E_{2}$ & $E_{2}^{*}=1, \quad E_{1}^{2}=1$ \\
\hline$E_{5}^{\hat{z}}=1$ & $E_{i}^{z}=E_{i}$ & $E_{3}^{2}=E_{2} E_{1}$ & $E_{\mathrm{a}}^{2}=1, \quad E_{1}^{2}=1$ \\
\hline$E_{\mathrm{5}}^{2}=1$ & $E_{4}^{2}=E_{2}$ & $E_{3}^{2}=E_{1}$ & $E_{2}^{2}=1, \quad E_{1}^{2}=1$ \\
\hline$E_{5}^{2}=E_{1}$ & $E_{4}^{2}=E_{2}$ & $E_{3}^{2}=E_{1}$ & $E_{2}^{2}=1, \quad E_{1}^{2}=1$ \\
\hline$E_{5}^{2}=1$ & $E_{4}^{2}=1$ & $E_{3}^{2}=1$ & $E_{2}^{2}=1, \quad E_{1}^{2}=1$ \\
\hline
\end{tabular}

36. Io ritorno all'ordine d'idee col quale ho incominciato il presente paragrafo e riscrivo le formole:

$$
E_{5}^{-1} E_{4} E_{5}=E_{4} E_{3}^{\prime}, \quad E_{5}^{-1} E_{3} E_{5}=E_{3} E_{2}^{\prime}, \quad E_{4}^{-1} E_{3} E_{4}=E_{3} \text {. }
$$

Ho già studiato in modo completo il caso in cui l'elemento $E_{3}^{\prime}$ appartiene al gruppo $H$, ed ora voglio fare l'ipotesi che il detto elemento stia fuori di $H$.

La potenza $E_{3}^{\prime}$ sta in $H$ e quindi si ha $E_{g}^{\prime p}=1$; inoltre, giacchè non pud $E_{2}^{\prime}$ coincidere con l'elemento identico perchè $E_{3}$ è fuori di $H$, posso scrivere le relazioni :

$$
E_{5}^{-1} E_{4} E_{5}=E_{4} E_{3}, \quad E_{5}^{-1} E_{3} E_{5}=E_{3} E_{4}, \quad E_{4}^{-1} E_{3} E_{4}=E_{3} .
$$

Si supponga $p>2$ : allora, siccome l'elemento $E_{4}^{p}$ sta (n. $\left.{ }^{\circ} 24\right)$ in $H$, deve essere $E_{3}^{p}=1$.

Si ammetta, in prima ipotesi, il gruppo $H$ ciclico: in tale caso, scegliendo $E_{2}$ in modo che sia $E_{2}^{p}=1$, ho:

$$
E_{5}^{p}=E_{2}^{r}, \quad E_{4}^{p}=E_{0}^{\beta}, \quad E_{3}^{p}=1, \quad E_{3}^{p}=E_{1}, \quad E_{1}^{p}=1 ;
$$

e, purchè si rammenti che gli elementi $E_{2}, E_{1}$ stanno in $H$, queste formole, insieme alle (8), definiscono un gruppo $G_{5}{ }^{2}$ qualunque siano gl'interi $\alpha$ e $\%$ 
Se chiamo $E_{5}$ ed $E_{4}$ gli elementi $E_{5} E_{2}^{h}$ ed $E_{4} E_{3}^{k}$ rispettivamente, i numeri $\alpha$ e $\beta$ si cambiano in $\alpha+p h$ e $\beta+p k$, e quindi $i$ detti numeri si debbono intendere determinati a meno di multipli di $p$.

Cio posto, avendosi una seconda base canonica:

$$
\mathbf{F}_{5}, \mathbf{E}_{4}, \mathbf{E}_{3}, \mathbf{E}_{2}, \mathbf{E}_{1},
$$

definita nello stesso modo della prima, se, nella maniera più generale, si ha:

deve essere:

$$
\mathbf{E}_{5} \equiv E_{5}^{n} E_{4}^{v}, \quad \mathbf{E}_{4} \equiv E_{4}^{s}, \quad\left(\bmod G_{3}\right)
$$

$$
\mathbf{F}_{3} \equiv E_{3}^{u s}, \quad \mathbf{F}_{2} \equiv E_{2}^{u^{3 s}}, \quad\left(\bmod G_{1}\right)
$$

ed il prodotto $u s$ deve necessariamente supporsi primo con $p$.

Intanto, dalle (8) si ricava:

$$
\left(E_{5} E_{4}\right)^{n}=E_{5}^{n} E_{4}^{n} E_{3}^{\left(\begin{array}{l}
n \\
2
\end{array}\right)} E_{2}^{\left(\begin{array}{l}
n \\
3
\end{array}\right)}
$$

e perciò, se ̀̀ $p>3$, si ha:

$$
\left(E_{5} E_{4}\right)^{p}=E_{5}^{p} E_{4}^{p}
$$

Allora, sia $p$ maggiore od eguale a 3 , eseguendo l'operazione:

$$
\left(\begin{array}{lllll}
E_{5}, & E_{4}, & E_{3}, & E_{2}, & E_{1} \\
\mathbf{E}_{5}, & \mathbf{E}_{4}, & \mathbf{I}_{3}, & \mathbf{E}_{2}, & \mathbf{E}_{4}
\end{array}\right)
$$

si vede subito che $\alpha$ e $\beta$ subiscono la sostituzione congrua:

$$
\begin{aligned}
& u^{2} s \alpha_{1} \equiv \alpha u+\beta v \\
& u^{2} \beta_{1} \equiv \beta
\end{aligned} \quad\{(\bmod p) .
$$

Dunque: se $\beta$ è un multiplo di $p$, posso determinare $u$ ed $s$ in modo che $\alpha_{1}$ sia 0 il resto 0 il resto 1 di $p$; se invece $\beta$ è primo con $p$, posso determinare $u$ in modo che sia $0 \beta_{1} \equiv 1(\bmod p)$ oppure $\beta_{1} \equiv \varepsilon(\bmod p) \mathrm{c}$, in entrambi i casi, dispongo di $v$ in modo che $\alpha_{1}$ sia il resto 0 di $p$.

In conclusione, quando $H$ è ciclico ed è $p>2$, si hanno quattro gruppi $G_{5}{ }^{2}$ in corrispondenza alle seguenti quattro serie di formole che sono modificazioni delle $(9)$.

$$
\begin{array}{|lllll}
E_{5}^{p}=1, & E_{4}^{p}=1, & E_{3}^{p}=1, & E_{2}^{p}=E_{1}, & E_{1}^{p}=1 \\
\hline E_{5}^{p}=E_{2}, & E_{4}^{p}=1, & E_{3}^{p}=1, & E_{2}^{p}=E_{1}, & E_{1}^{p}=1 \\
\hline E_{5}^{p}=1, \quad E_{4}^{p}=E_{2}, & E_{3}^{p}=1, & E_{2}^{p}=E_{1}, & E_{1}^{p}=1 \\
\hline E_{5}^{p}=1, & E_{4}^{p}=E_{2}^{e}, & E_{3}^{p}=1, & E_{2}^{p}=E_{1}, & E_{1}^{p}=1
\end{array}
$$


Il $G_{5}{ }^{2}$ corrispondente alla prima serie di formole è un gruppo $G_{5}{ }^{2,3}$, ed il relativo gruppo $K$ è generato dagli elementi $E_{3}, E_{1}$; invece i tre gruppi corrispondenti alle ultime tre formole sono gruppi $G_{5}^{2,2}$ e, per ciascuno di essi, il gruppo $K$ è generato dagli elementi $E_{3}^{\prime}, E_{2}, E_{1}$.

37. Si ammetta, in seconda ipotesi, che il gruppo $H$ abbia tutti i suoi elementi di grado $p$.

In tale caso bisogna sostituire le (9) con le formole:

$$
E_{5}^{p}=E_{2}^{i} E_{1}^{p}, \quad E_{1}^{n}=E_{2}^{\prime} E_{1}^{\delta}, \quad E_{3}^{p}=1, \quad E_{2}^{p}=1, \quad E_{1}^{p}=1,
$$

le quali, insieme alle (8), definiscono un gruppo $G_{5}^{2}$ qualunque siano gl'interi $\alpha, \beta, \gamma, \delta$.

Se il gruppo $G_{5}^{2}$ così definito è un $G_{5}^{2,2}$, uno almeno dei due numeri $\alpha, \gamma$ è primo con $p$.

Quando è $\gamma$ primo con $p$, io pongo a definizione dell'elemento $E_{2}$ la relazione $E_{4}^{p}=E_{2}$. Allora, se $v$ ed $s$ sono interi ed il secondo è primo con $p$, eseguendo l'operazione:

$$
\left(\begin{array}{ccccc}
E_{5}, & E_{1}, & E_{3}, & E_{2}, & E_{1} \\
E_{5} E_{1}^{v}, & E_{4}^{s}, & E_{3}^{s} E_{1}^{s}, & E_{3}^{s}, & E_{1}^{s}
\end{array}\right)
$$

la quale non altera nè la detta relazione nè le formole (8), si vede subito che i numeri $\alpha, \beta$ si portano in due numeri $\alpha_{1}, \beta_{1}$ tali che:

ovvero tali che:

$$
s \alpha_{1} \equiv \alpha+v, \quad s \beta_{1} \equiv \beta,(\bmod p>3),
$$

$$
s \alpha_{1} \equiv \alpha+v, \quad s \beta_{1} \equiv \beta+v, \quad(\bmod p=3) .
$$

Dunque, in entrambi i casi, disponendo opportunamente di $v$ ed $s$ si può portare la coppia $(\alpha \beta)$ o in $(01)$ ovvero in $(00)$.

Quando è $\gamma$ un multiplo di $p$ ma è $\alpha$ primo con $p$, io pongo a definizione dell'elemento $E_{2}$ la relazione $E_{5}^{\prime \prime}=E_{2}^{\prime}$. Allora, se $u$ è un intero primo con $p$, eseguendo l'operazione:

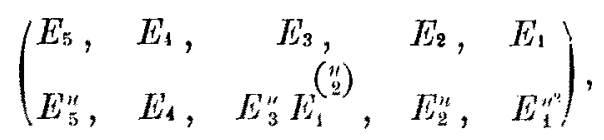

la quale non altera nè la detta relazione nè le formole (8), si ha:

$$
u^{2} \partial_{1} \equiv \partial,(\bmod p) \text {. }
$$

Dunque, disponendo convenientemente del numero intero $u$, si può portare $\delta$ in uno dei tre resti $\varepsilon, 1,0$ relativi a $p$ ed, evidentemente, uno qualunque di questi casi esclude gli altri due. 
In conclusione, continuando a supporre $p>2$, i gruppi $G_{5}{ }^{2,2}$ definiti dalle relazioni (8) e (10) sono cinque e corrispondono alle seguenti modificazioni delle formole $(10)$.

\begin{tabular}{|lllll}
$E_{5}^{p}=E_{1}$, & $E_{4}^{p}=E_{2}$, & $E_{3}^{p}=1$, & $E_{2}^{p}=1$, & $E_{1}^{p}=1$ \\
\hline$E_{5}^{p}=1$, & $E_{1}^{p}=E_{2}$, & $E_{3}^{p}=1$, & $E_{2}^{p}=1$, & $E_{1}^{p}=1$ \\
\hline$E_{5}^{p}=E_{2}$, & $E_{4}^{p}=1$, & $E_{3}^{p}=1$, & $E_{2}^{p}=1$, & $E_{1}^{p}=1$ \\
\hline$E_{5}^{p}=E_{2}$, & $E_{1}^{p}=E_{1}$, & $E_{3}^{p}=1$, & $E_{2}^{p}=1$, & $E_{1}^{p}=1$ \\
\hline$E_{5}^{p}=E_{2}$, & $E_{1}^{p}=E_{1}^{\varepsilon}$, & $E_{3}^{p}=1$, & $E_{2}^{p}=1$, & $E_{1}^{p}=1$ \\
\hline
\end{tabular}

Se il gruppo definito dalle relazioni (8) e (10) è un $G_{5}{ }^{2,3}$, i due numeri $\alpha, \gamma$ sono multipli di $p$; allora le dette relazioni mostrano che il gruppo $G_{5}{ }^{2,3}$, così definito, si ottiene aggiungendo in modo ovvio l'elemento $E_{2}$ al gruppo $G_{4}^{2,2}$ generato dagli elementi $E_{5}, E_{4}, E_{3}, E_{1}$. Le formole di composizione dei gruppi $G_{4}^{2,2}$ sono le (16) del $\S$ II nel caso di $p>3$ e le (17) dello stesso paragrafo nel caso di $p=3$; quindi, riscrivendo le dette formole dopo avere osservato che gli elementi che vi figurano $E_{4}, E_{3}, E_{2}$ hanno ora, ordinatamente, i nomi $E_{5}, E_{4}, E_{s}$, ed aggiungendo la relazione $E_{2}^{p}=1$, si ottengono tutte le modificazioni delie (10) che corrispondono a gruppi $G_{5}{ }^{2,3}$ distinti.

Dunque, nelle attuali ipotesi, se è $p>3$, si hanno quattro gruppi $G_{5}{ }^{2,3}$ corrispondenti alle formole:

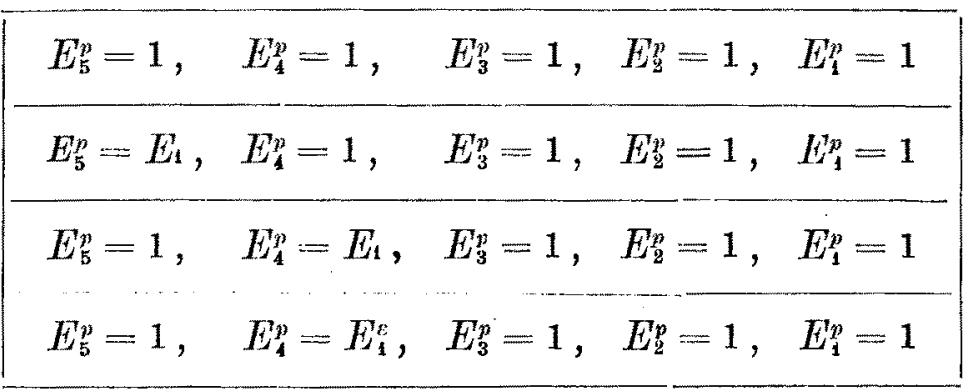

e se ̀̀ $p=3$, si hanno pure quattro gruppi $G_{5}^{2,3}$ corrispondenti alle 
formole :

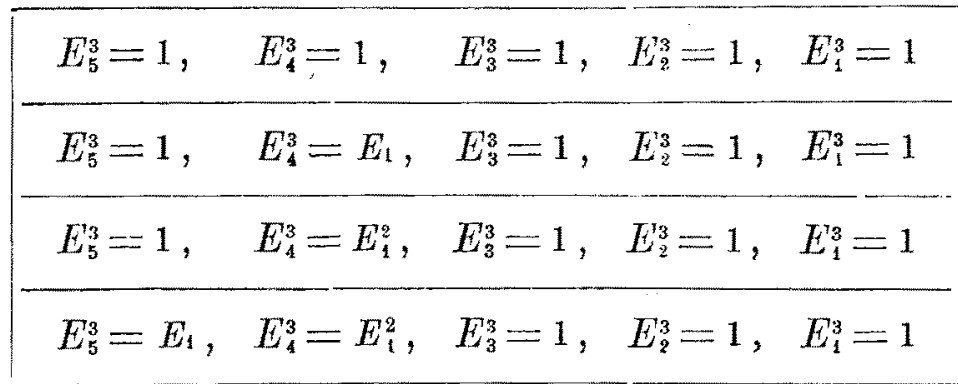

38. Mi rimane a considerare il caso di $p=2$ che ho escluso al principio del n. ${ }^{\circ} 36$.

Giacchè un gruppo $G_{5}{ }^{2}$ non può avere che un solo divisore Abeliano $\mathrm{d}$ 'indice $p$, risulta che, nella presente ipotesi di $p=2$, il quadrato dell' elemento $I_{5}$ che figura nelle relazioni (8) deve appartenere necessariamente al gruppo $H$. Intanto le dette relazioni dànno:

$$
E_{5}^{-2} E_{4} E_{5}^{2}=E_{4} E_{3}^{2} E_{4}^{\prime}
$$

e percio deve essere $E_{3}^{2}=E_{1}$. Allora la prima delle (8) mostra che la potenza $E_{1}^{z}$ è fuori di $H$ e quindi essa coincide con uno dei quattro elementi:

$$
E_{3}, \quad E_{3} E_{2}, \quad E_{3} E_{1}, \quad E_{3} E_{2} E_{1} \text {. }
$$

Io suppongo che si avveri il primo od il secondo caso, perchè, se fosse altrimenti, assumerei come elemento $E_{4}$ l'elemento $E_{4} E_{3}$ e quindi come elemento $E_{3}^{\prime}$ l'elemento $E_{3}^{\prime} E_{1}$.

Ciò posto, si dimostra col solito procedimento che, aggiungendo alle (8) le relazioni :

$$
E_{5}^{\prime 2}=E_{2}^{\prime t} E_{1}^{\prime}, \quad E_{1}^{\prime 2}=E_{3}^{\prime}, \quad E_{3}^{\prime 2}=E_{1}^{\prime}, \quad E_{2}^{2}=E_{1}^{\prime h}, \quad E_{1}^{\prime}=1,
$$

oppure le relazioni :

$$
E_{5}^{\prime \prime}=E_{2}^{\prime \prime} E_{1}^{\prime}, \quad E_{4}^{\prime 2}=E_{3}^{\prime} E_{2}^{\prime}, \quad E_{3}^{\prime}=E_{1}^{\prime}, \quad E_{2}^{2}=E_{1}^{\prime h}, \quad E_{1}^{2}=1,
$$

si definisce in ogni caso un gruppo $G_{5}^{2}$ di grado 32.

Essendo, in virtù delle (8),

$$
\left(E_{5} E_{4}\right)^{2}=E_{5}^{2} E_{4}^{2} E_{3}
$$

si vede che, chiamando $E_{5}$ l'elemento $E_{5} E_{4}$, se hanno luogo le $(11)$, gl'interi $\alpha$ e $\beta$ si cambiano rispettivamente in $\alpha$ e $\beta+1$, e, se hanno luogo le (12), i detti interi si cambiano rispettivamente in $\alpha+1$ e $\beta+1$. 
Dunque, nelle (11) io posso supporre che sia $E_{5}^{9}=1$ ovvero $E_{5}^{2}=E_{2}$, ed è poi facile convincersi che questi due casi non si possono ridurre l'uno nell' altro qualunque sia $h$.

Nelle (12) posso supporre che sia $E_{5}^{2}=1$ ovvero $E_{5}^{2}=E_{1}$, e questi due casi sono tra loro irreduttibili soltanto quando ̀̀ $h=0$; ma, se $\grave{\mathrm{e}} h=1$, i detti casi si possono ridurre l'uno nell'altro chiamando $E_{5}$ l'elemento $E_{5} E_{2}$.

In conclusione, nell'attuale jpotesi, si hanno sette gruppi $G_{5}{ }^{2}$ di grado 32 , i quali sono definiti dalle (8) insieme alle seguenti relazioni :

\begin{tabular}{|c|c|c|c|c|}
\hline$E_{5}^{2}=1$ & $E_{4}^{2}=E_{3}$ & $E_{3}^{2}=E_{1}$ & $E_{2}^{2}=1$ & $E_{1}^{2}=1$ \\
\hline$E_{5}^{2}=1$ & $E_{4}^{2}=E_{3}^{j}$ & $E_{3}^{2}=E_{1}$ & $E_{2}^{2}=E_{1}$ & $E_{1}^{2}=1$ \\
\hline$E_{5}^{2}=E_{2}$ & $E_{4}^{2}=E_{3}$ & $E_{3}^{2}=E_{1}$ & $E_{2}^{2}=1$ & $E_{1}^{2}=1$ \\
\hline$E_{5}^{2}=E_{2}$ & $E_{4}^{2}=E_{3}$ & $E_{3}^{2}=H_{1}$ & $E_{2}^{2}=E_{\mathrm{t}}$ & $E_{1}^{2}=1$ \\
\hline$E_{\mathrm{5}}^{2}=1$ & $E_{4}^{2}=E_{3} E_{2}$ & $E_{3}^{2}=H_{1}$ & $E_{2}^{2}=1$ & $E_{1}^{2}=1$ \\
\hline$E_{5}^{2}=1$ & $E_{4}^{9}=E_{3} E_{2}$ & $E_{3}^{2}=E_{1}$ & $E_{2}^{\mathrm{a}}=E_{\mathrm{t}}$ & $E_{1}^{2}=1$ \\
\hline$E_{5}^{\mathrm{v}}=E_{1}$ & $E_{4}^{9}=E_{3} E_{2}$ & $E_{3}^{2}=E_{1}$ & $E_{2}^{2}=1$ & $E_{1}^{2}=1$ \\
\hline
\end{tabular}

I due gruppi, che corrispondono alle prime due serie di formole, sono gruppi $G_{\mathrm{s}}^{2,3}$; invece i cinque gruppi corrispondenti alle ultime cinque formole sono gruppi $G_{5}^{2,2}$.

39. Io riepilogo brevemente i risultati ottenuti nel presente paragrafo.

I gruppi $G_{5}^{2}$, che posseggono un divisore Abeliano d'indice $p$, si dividono in due categorie, secondo che il gruppo $K$ coincide ovvero non coincide col gruppo $H$.

Nella prima categoria esistono $p+8$, e non più, gruppi $G_{5}{ }^{2}$ di grado $p^{5}$ con $p>2$, e soltanto nove gruppi $G_{5}{ }^{2}$ di grado 32 .

Tutti i gruppi della prima categoria sono gruppi $G_{5}{ }^{2,3}$.

Nella seconda categoria esistono soltanto tredici gruppi $G_{5}{ }^{2}$ di grado $p^{5}$ con $p>2$ : di questi tredici gruppi, cinque sono gruppi $G_{5}^{2,3}$ ed i rimanenti otto sono gruppi $G_{5}^{2,2}$. 
La detta seconda categoria contiene ancora sette gruppi $G_{5}{ }^{2}$ di grado 32 : di questi gruppi, due sono gruppi $G_{5}{ }^{2,3}$ ed i rimanenti cinque sono gruppi $G_{5}{ }^{p, 2}$.

Tutti i gruppi che sono stati trovati in questo paragrafo sono rappresentati nelle seguenti tabelle.

I.

$$
\begin{gathered}
\left\{H\left(E_{2}, E_{4}\right)|;| K=H \mid\right. \\
E_{5}^{-1} E_{4} E_{5}=E_{4} E_{2}, \quad E_{5}^{-1} E_{3} E_{5}=E_{3} E_{1}, \quad E_{4}^{-1} E_{3} E_{4}=E_{3} .
\end{gathered}
$$

\begin{tabular}{|c|c|c|c|c|c|c|}
\hline & $E_{5}^{p}$ & $E_{4}^{p}$ & $E_{3}^{p}$ & $E_{z}^{p}$ & $E_{1}^{p}$ & \\
\hline \multirow{11}{*}{ 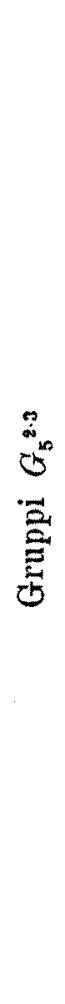 } & 1 & $E_{2}^{\varepsilon^{n n_{-1}}}$ & $E_{1}^{\varepsilon^{\prime \prime \prime+1}}$ & 1 & 1 & \multirow{3}{*}{$\frac{310}{ \pm}$} \\
\hline & 1. & $E_{2}^{-1}$ & $E_{1}$ & 1 & 1 & \\
\hline & $E_{2}$ & 1 & $E_{1}$ & 1 & 1 & \\
\hline & 1 & $E_{2} E_{1}^{\prime}$ & $E_{2} E_{1}^{\varepsilon^{2 m+1}}$ & 1 & 1 & \multirow{8}{*}{$\widehat{\sqrt{210}}$} \\
\hline & 1 & $E_{1}$ & $E_{z}^{*}$ & 1 & 1 & \\
\hline & 1 & $E_{2}$ & $E_{1}$ & 1 & 1 & \\
\hline & 1 & $E_{2}$ & $E_{2} E_{1}$ & 1 & 1 & \\
\hline & 1 & 1 & $E_{2}$ & 1 & 1 & \\
\hline & $E_{1}$ & 1 & $E_{2}$ & 1 & 1 & \\
\hline & $E_{1}$ & 1 & 1 & 1 & 1 & \\
\hline & 1 & 1 & 1 & 1 & 1 & \\
\hline & \multicolumn{5}{|c|}{$m=0, \ldots, \frac{p-3}{2}\{p>2\}$} & \\
\hline
\end{tabular}


il cui grado è la quinta potenza di un numero primo.

II.

Gruppi $G_{5}{ }^{2}$ di grado 32.

$\left.\left\{H\left(E_{2}, E_{1}\right)\right\} ; \quad K=H\right\}$.

$E_{5}^{-1} E_{4} E_{5}=E_{4} E_{2}, \quad E_{5}^{-1} E_{3} E_{5}=E_{3} E_{1}, \quad E_{4}^{-1} E_{3} E_{4}=E_{3}$.

\begin{tabular}{|c|c|c|c|c|c|}
\hline & $E_{5}^{2}$ & $E_{1}^{2}$ & $E_{3}^{2}$ & $E_{2}^{2}$ & $E_{1}^{2}$ \\
\hline \multirow{9}{*}{ 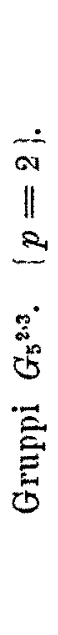 } & 1 & 1 & $E_{1}$ & 1 & 1 \\
\hline & $E_{\mathrm{t}}$ & 1 & $E_{t}$ & 1 & 1 \\
\hline & 1 & 1 & $E_{2}$ & 1 & 1 \\
\hline & 1 & $E_{t}$ & $E_{2}$ & 1 & 1 \\
\hline & $E_{t}$ & $E_{1}$ & $E_{2}$ & 1 & 1 \\
\hline & 1 & $E_{1}$ & $E_{2} E_{1}$ & 1 & 1 \\
\hline & 1 & $E_{2}$ & $E_{i}$ & 1 & 1 \\
\hline & $E_{1}$ & $E_{2}$ & $E_{\mathrm{t}}$ & 1 & 1 \\
\hline & 1 & 1 & 1 & 1 & 1 \\
\hline
\end{tabular}

$\left\{H\left(E_{\mathrm{q}}, E_{\mathrm{t}}\right)\right\} ; \quad\{K=H\}$.

$E_{5}^{-1} E_{4} E_{5}=E_{4} E_{3}, \quad E_{5}^{-1} E_{3} E_{j}=E_{3} E_{1}, \quad E_{4}^{-1} E_{3} E_{4}=E_{3}$.

\begin{tabular}{|c|c|c|c|c|c|}
\hline & $E_{5}^{2}$ & $E_{4}^{2}$ & $E_{3}^{2}$ & $X_{2}^{2}$ & $E_{\mathrm{t}}^{q}$ \\
\hline \multirow{2}{*}{ 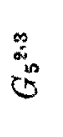 } & 1 & $E_{3}$ & $E_{1}$ & 1 & 1 \\
\hline & 1 & $E_{3}$ & $E_{1}$ & $E_{1}$ & 1 \\
\hline \multirow{5}{*}{ 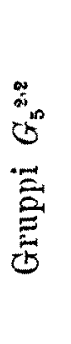 } & $E_{2}$ & $E_{3}$ & $E_{1}$ & 1 & 1 \\
\hline & $E_{u}$ & $E_{3}$ & $\overline{E_{1}}$ & $E_{1}$ & 1 \\
\hline & 1 & $E_{3} E_{2}$ & $E_{1}$ & 1 & 1 \\
\hline & 1 & $L_{3} E_{2}$ & $E_{1}$ & $E_{1}$ & 1 \\
\hline & $L_{1}$ & $E_{3} E_{2}$ & $F_{1}$ & 1 & 1 \\
\hline
\end{tabular}


III.

$\left\{H\left(E_{2}, E_{4}\right)\right\} ; \quad\{K=\mid=H\}$

$E_{5}^{-1} E_{4} E_{5}=E_{4} E_{3}, \quad E_{5}^{-1} E_{3} E_{3}=E_{3} E_{1}, \quad E_{4}^{-1} E_{3} E_{4}=E_{3}$.

\begin{tabular}{|c|c|c|c|c|c|c|}
\hline & $E_{\mathbf{5}}^{p}$ & $E_{4}^{p}$ & $E_{3}^{p}$ & $E_{2}^{p}$ & $E_{1}^{p}$ & \\
\hline \multirow{9}{*}{ 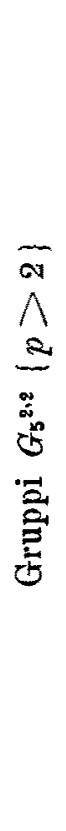 } & 1 & 1 & 1 & $E_{4}$ & 1 & $p \geqq 3$ \\
\hline & $E$ & 1 & 1 & $E_{1}$ & 1 & $p \geqq 3$ \\
\hline & 1 & $E_{2}$ & 1 & $E_{1}$ & 1 & $p \geqq 3$ \\
\hline & 1 & $E_{\mathbf{2}}^{\varepsilon}$ & 1 & $E_{1}$ & 1 & $p \geqq 3$ \\
\hline & $E_{\mathrm{l}}$ & $E_{2}$ & 1. & 1 & 1 & $p \geqq 3$ \\
\hline & 1 & $E_{\mathrm{g}}$ & 1 & 1 & 1 & $p \geqq 3$ \\
\hline & $E_{2}$ & 1 & 1 & 1 & 1 & $p \geqq 3$ \\
\hline & $E_{2}$ & $E_{1}$ & 1 & 1 & 1 & $p \geq 3$ \\
\hline & $E_{2}$ & $E_{1}^{\varepsilon}$ & 1 & 1 & 1 & $p \geq 3$ \\
\hline \multirow{5}{*}{ 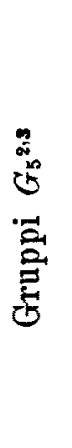 } & 1 & 1 & 1 & 1 & 1 & $p \geq 3$ \\
\hline & $E_{1}$ & 1 & 1 & 1 & 1 & $p>3$ \\
\hline & 1 & $E_{1}$ & 1 & 1 & 1 & $p \geq 3$ \\
\hline & 1 & $E_{1}^{\varepsilon}$ & 1 & 1 & 1 & $p>3$ \\
\hline & $E_{4}$ & $E_{1}^{2}$ & 1 & 1 & 1 & $p=3$ \\
\hline
\end{tabular}




\section{$\S$ VI. I Gruppi $G_{5}^{3,2}$.}

40. In un gruppo $G_{5}{ }^{3}$ il divisore invertibile $H$ è di grado $p$ ed è quindi contenuto in ogni divisore normale di $G_{5}{ }^{3}$. Nel presente paragrafo mi propongo di trovare tutti i gruppi $G_{5}{ }^{3}$ ehe hanno due soli divisori indipendenti d'indice $p$ : distinguerò questi gruppi in due categorie, ascrivendo alla prima categoria ogni gruppo $G_{5}^{3,2}$, che ammette un divisore Abeliano d'indice $p$, ed alla seconda categoria ogni gruppo $G_{5}{ }^{3,2}$ che non possiede un tale divisore.

Se :

$$
G_{5}^{3,2}, G_{1}{ }^{0}, G_{3}, G_{2}, G_{1},
$$

è una serie canonica di composizione di un gruppo della prima categoria ed:

$$
E_{5}, E_{4}, E_{3}, E_{2}, E_{1},
$$

è una base relativa alla detta serie si ha:

$$
\begin{array}{lll}
E_{5}^{\prime-1} E_{4} E_{5}=E_{4} E_{3}^{\prime}, & E_{5}^{-1} E_{3}^{\prime} E_{5}=E_{3}^{\prime} E_{2}^{\prime}, \quad E_{5}^{-1} E_{2} E_{5}=E_{2} E_{4}^{\prime}, \\
E_{4}^{\prime}{ }^{-1} E_{3}^{\prime} E_{4}^{\prime}=E_{3}, & E_{4}^{-1} E_{2} E_{4}=E_{2}, \quad E_{3}^{\prime-1} E_{2}^{\prime} E_{3}^{\prime}=E_{2} .
\end{array}
$$

Qualsiasi elemento di $G_{4}{ }^{0}$ che è invertibile con $E_{5}$ appartiene necessariamente ad $H$ : infatti, un tale elemento risulta invertibile con ogni altro elemento di $G_{5}^{3,2}$.

Ciò posto, l'elemento $E_{1}^{\prime}$ che figura nelle formole precedenti è un elemento generatore $E_{1}$ del gruppo $G_{1}=H$.

L'elemento $E_{2}^{\prime}$ è fuori di $G_{1}$, perchè, se fosse $E_{2}^{\prime}=E_{1}^{\alpha}$, l'elemento $E_{3} E_{2}^{-\alpha}$, che è fuori di $H$, risulterebbe invertibile con $E_{5}^{\prime}$. Suppongo dunque $E_{2}^{\prime}=E_{2}$.

L'elemento $E_{3}^{\prime}$ è fuori di $G_{2}$, perchè, se fosse $E_{3}^{\prime}=E_{8}^{\prime \prime} E_{1}^{\beta}$, l' elemento $E_{4} E_{3}^{-\alpha} E_{a^{-i}}$, che è fuori di $H$, risulterebbe invertibile con $E_{5}$. Suppongo dunque $E_{3}^{\prime}=E_{3}$.

Io posso ora scrivere le formole definitive:

$$
\left.\begin{array}{lll}
E_{5}^{-1} E_{4} E_{5}=E_{4} E_{3}, & E_{5}^{-1} E_{3} E_{5}=E_{3} E_{2}, & E_{5}^{-1} E_{2} E_{5}=E_{2} E_{1}, \\
E_{4}^{-1} E_{3} E_{4}=E_{3}, & E_{4}^{1} E_{2} E_{4}=E_{2}, \quad E_{3}{ }^{1} E_{2} E_{3}=H_{2},
\end{array}\right\}
$$

le quali mostrano che il gruppo $G_{3}$ coincide necessariamente con $K$; inoltre, il ragionamento che ho fatto per stabilire le (1) prova che ogni gruppo $G_{5}{ }^{3}$, che possiede un divisore Abeliano d'indice $p$, è certamente un gruppo $G_{\mathbf{b}}{ }^{3,2}$. 
E importante osservare che la potenza $E_{5}^{n}$ appartiene al gruppo $G_{3}$ ed è invertibile con $E_{5}$ : dunque la detta potenza sta in $H$.

Dalle relazioni (1) si ricava:

$$
E_{5}^{-n} E_{4} E_{5}^{n}=E_{4} E_{3}^{n} E_{2}^{\left(\begin{array}{l}
n \\
2
\end{array}\right)} E_{1}^{\left(\begin{array}{l}
n \\
3
\end{array}\right)}, \quad E_{5}^{-n} E_{3} E_{5}^{n}=E_{3} E_{2}^{n} E_{1}^{\left(\begin{array}{l}
n \\
2
\end{array}\right)}
$$

quindi, supposto $p>3$, per l'osservazione precedente deve essere:

Allora, siccome è :

$$
E_{2}^{p}=1, \quad E_{3}^{p}=1 \text {. }
$$

$$
E_{5}^{-1} E_{4}^{p} E_{5}=E_{4}^{p} E_{3}^{p}
$$

l'elemento $E_{4}^{p}$ risulta invertibile con $E_{5}$ e perciò sta in $H$.

Si hanno dunque le formole:

$$
E_{5}^{p}=E_{1}^{\alpha}, \quad E_{4}^{p}=E_{1}^{\beta}, \quad E_{3}^{p}=1, \quad E_{2}^{p}=1, \quad E_{\mathrm{i}}^{p}=1,
$$

le quali, insieme alle (1) definiscono un gruppo $G_{5}^{3,2}$ qualunque siano gl' interi $\alpha, \beta$ purchè si tenga presente che $E_{1}$ appartiene ad $H$ e chè è $p>3$.

Sia ora:

$$
\mathbf{E}_{5}, \quad \mathbf{E}_{4}, \quad \mathbf{E}_{3}, \quad \mathbf{E}_{2}, \quad \mathbf{E}_{4}
$$

una seconda base canonica di $G_{5}^{3,2}$ definita come la prima. Ponendo, nel modo più generale,

$$
\mathrm{r}_{\mathrm{s}} \equiv E_{\mathrm{t}}^{u} E_{1}^{v}, \quad \mathrm{I}_{4} \equiv E_{4}^{s}, \quad\left(\bmod G_{3}\right),
$$

si trova successivamente:

$$
\mathbf{E}_{3} \equiv E_{3}^{u s}\left(\bmod G_{2}\right), \quad \mathbf{E}_{2} \equiv E_{z}^{u^{u_{s}}}\left(\bmod G_{1}\right), \quad \mathbf{E}_{1} \equiv E_{1}^{u^{i s},},
$$

e quindi il prodotto $u s$ deve supporsi primo con $p$.

Dopo cio, pensando alle (1), dopo un breve calcolo si ha:

$$
\left(E_{5} E_{4}\right)^{n}=E_{5}^{n} E_{4}^{n} E_{3}^{\left(\begin{array}{l}
n \\
2
\end{array}\right)} E_{2}^{\left(\begin{array}{l}
n \\
3
\end{array}\right)} E_{1}^{\left(\begin{array}{l}
n \\
4
\end{array}\right)}
$$

dunque, avendo supposto $p>3$, risulta:

e quindi :

$$
\left(E_{\mathrm{b}} E_{4}\right)^{p}=E_{5}^{p} E_{4}^{r}
$$

$$
\mathbf{E}_{5}^{\prime \prime}=E_{5}^{p u} E_{4}^{\prime v}=E_{1}^{\alpha u+\beta v} \text {. }
$$

Si vede allora immediatamente che l'operazione:

$$
\left(\begin{array}{lllll}
E_{5}, & E_{1}, & E_{3}, & E_{2}, & E_{1} \\
\mathbf{E}_{5}, & \mathbf{E}_{4}, & \mathbf{E}_{3}, & \mathbf{E}_{9}, & \mathbf{E}_{4}
\end{array}\right),
$$


porta i numeri $\alpha$ e $\beta$ nei numeri $\alpha_{1}$ e $\beta_{1}$ tali che:

$$
\begin{array}{rlrl}
u^{3} s \alpha_{1} & \equiv a u+\beta v, & \\
u^{3} \beta_{1} & \equiv \beta .
\end{array}
$$

Qui bisogna distinguere il caso in cui $p-1$ è primo con 3 dal caso in cui $p-1$ è un multiplo di 3 . Nel primo caso, se non è $\beta$ un multiplo di $p$, si può scegliere $u$ in modo che sia $u^{3} \equiv \beta(\bmod p)$ : quindi $\beta_{1}$ si può portare 0 nel resto 1 oppure nel resto 0 di $p$; invece, nel secondo caso, il numero $\beta_{1}$ si può portare in uno dei quattro resti $0,1, \varepsilon, \varepsilon^{2}$ di $p$, ed una qualunque di queste quattro ipotesi esclude le altre tre. Inoltre si osservi che tutte le volte che è $\beta$ primo con $p$, disponendo convenientemente di $v$, posso portare $\alpha_{1}$ nel resto 0 di $p$; e se è $\beta$ un multiplo di $p$, disponendo convenientemente di $s$ posso portare $\alpha_{1}$ in uno dei due resti 1,0 di $p$.

In conclusione, se è $p-1$ primo con 3 , esistono solamente tre gruppi $G_{5}{ }^{3,2}$ che hanno ciascuno un divisore Abeliano d'indice $p$, e questi tre gruppi corrispondono alle seguenti modificazioni delle formole (2):

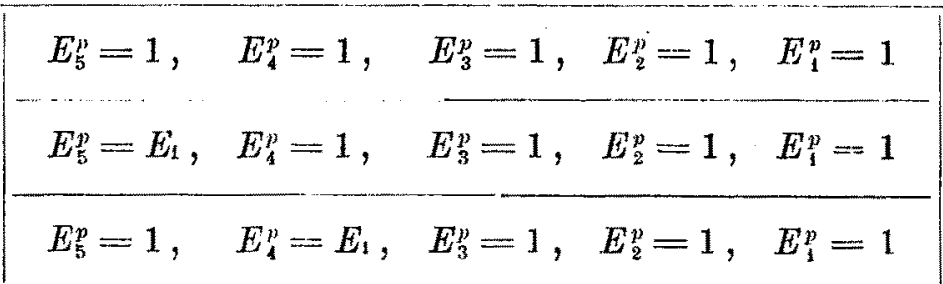

Se poi $p-1$ è un multiplo di 3 , oltre ai precedenti tre gruppi, si hanno ancora due nuovi gruppi $G_{5}{ }^{3,2}$ che corrispondono alle seguenti modificazioni delle. (2) :

$$
\begin{aligned}
& E_{5}^{p}=1, \quad E_{4}^{p}=E_{1}^{r}, \quad E_{3}^{p}=1, \quad E_{2}^{p}=1, \quad E_{1}^{p}=1 \\
& \hline E_{5}^{p}=1, \quad E_{4}^{p}=E_{1}^{c^{2}}, \quad E_{3}^{p}=1, \quad E_{2}^{p}=1, \quad E_{1}^{p}=1
\end{aligned}
$$

41. Supponendo ora $p=3$, le (1) danno:

$$
E_{5}^{-3} E_{4} E_{5}^{3}=E_{4} E_{3}^{3} E_{4}^{3} E_{4}, \quad E_{5}^{-3} E_{3} E_{5}^{3}=E_{3} E_{2}^{3} ;
$$

juindi, giacchè $E_{5}^{3}$ sta in $H$, si ha successivamente:

$$
E_{2}^{3}=1, \quad E_{3}^{3}=E_{1}^{9} \text {. }
$$


Allora le formole (1) e la relazione:

$$
E_{5}^{-1} E_{4}^{3} E_{5}=E_{4}^{3} E_{3}^{3}=E_{4}^{3} E_{4}^{2},
$$

mostrano che l'elemento $E_{4}$ deve coincidere con un elemento di $G_{2}$ del tipo $E_{1}^{2} E_{1}^{p}$.

Si hanno dunque le formole:

$$
E_{\mathrm{s}}^{3}=E_{\mathrm{i}}^{(t,}, \quad E_{\mathrm{4}}^{3}=E_{\mathrm{2}}^{2} E_{\mathrm{1}}^{3}, \quad E_{3}^{3}=E_{1}^{2}, \quad E_{\mathrm{2}}^{3}=1, \quad E_{1}^{3}=1,
$$

le quali, insieme alle (1), definiscono sempre un gruppo $G_{5}^{3,2}$ di grado 243.

Si osservi che l'operazione:

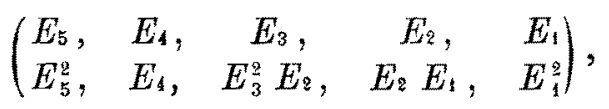

lascia inalterate le (1) e cambia o e $\beta$ rispettivamente in o e $2 \beta+2$ : dunque si può ritenere o $\beta=0$ oppure $\beta=1$. Se è $\beta=0$, osservando che è :

$$
\left(E_{5} E_{4}^{3}\right)^{3}=E_{5}^{3} E_{4}^{3} E_{3}^{3} E_{2}=E_{4}^{c+2},
$$

si vede che, chiamando $E_{5}^{\prime}$ uno dei due elementi $E_{5} E_{4}, E_{5} E_{4}^{2}$, si può supporre $\alpha=0$. $\mathrm{Ma}$, se ̀̀ $\beta=1$, è facile verificare che, cambiando comunque la base canonica, in modo tale però che le (1) si conservino, i numeri a e $\beta$ restano inalterati: dunque, se è $\beta=1$, le tre ipotesi $\alpha=0,1,2$ non si possono ridurre una in un'altra.

In conclusione, esistono soltanto quattro gruppi $G_{5}^{3,2}$ di grado 243 che posseggono ciascuno un divisore Abeliano di grado 81, e questi quattro grupp; sono definiti dalle (1) e dalle seguenti modificazioni delle formole (3):

$$
\begin{array}{|lll}
E_{5}^{3}=1, \quad E_{4}^{3}=E_{2}^{2}, & E_{3}^{3}=E_{1}^{2}, \quad E_{2}^{3}=1, \quad E_{1}^{3}=1 \\
E_{5}^{3}=1, \quad E_{1}^{3}=E_{2}^{2} E_{1}, & E_{3}^{3}=E_{1}^{2}, & E_{2}^{3}=1, \quad E_{1}^{3}=1 \\
E_{5}^{3}=E_{1}, \quad E_{4}^{3}=E_{0}^{2} E_{1}, & E_{3}^{3}=E_{1}^{2}, & E_{2}^{3}=1, \quad E_{1}^{3}=1 \\
E_{5}^{3}=E_{1}^{2}, \quad E_{4}^{3}=E_{2}^{2} E_{1}, & E_{3}^{3}=E_{1}^{2}, & E_{2}^{3}=1, \quad E_{1}^{3}=1
\end{array}
$$

42. Io suppongo finalmente $p=2$.

Dalle (1) si ricava:

$$
E_{5}^{-2} F_{4} E_{5}^{2}=E_{4} E_{3}^{4} E_{2}, \quad E_{5}^{-2} E_{3}^{\prime} E_{5}^{2}=E_{3} E_{2}^{2} E_{1}
$$


quindi, giacchè $E_{5}^{2}$ sta in $H$, si ha successivamente :

$$
E_{2}^{2}=E_{1}, \quad E_{3}^{2}=E_{\mathrm{q}}^{-1} .
$$

Allora le formole (1) e la relazione:

$$
E_{5}^{-1} E_{4}^{2} E_{5}^{2}=E_{4}^{2} E_{3}^{2}=E_{4}^{2} E_{9}^{-1} \text {, }
$$

mostrano che l'elemento $E_{4}^{2}$ deve coincidere con un elemento di $G_{3}$ del tipo $E_{3}^{-1} E_{1}^{*}$.

Si hanno dunque le formole:

$$
E_{5}^{2}=E_{1}^{\prime \prime}, \quad E_{4}^{9}=E_{3}^{-1} E_{1}^{i}, \quad E_{3}^{2}=E_{3}^{-1}, \quad E_{3}^{2}=E_{1}^{\prime}, \quad E_{1}^{2}=1,
$$

le quali, insieme alle (1) definiscono sempre un gruppo $G_{5}^{3,2}$ di grado 32.

Ciò posto, si verifica facilmente che, cambiando comunque la base canonica in modo tale però che le (1) si conservino, il numero $\beta$ resta sempre inalterato ed il numero $\alpha$ o resta inalterato oppure si cambia in $\alpha+\beta$.

Dunque, se ̀̀ $\beta=0$, le due ipotesi $\alpha=0,1$ non si possono ridurre l'una nell'altra; ma, se è $\beta=1$, si può sempre supporre $\alpha=0$.

In conclusione, esistono soltanto tre gluppi $G_{: 5}^{3,2}$ di grado 32 che posseggono ciascuno un divisore Abeliano di grado 16, e questi tre gruppi sono definiti dalle formole (1) e dalle seguenti inodificazioni delle (4):

$$
\begin{array}{lll}
E_{5}^{2}=1, \quad E_{4}^{2}=E_{3}^{-1}, & E_{3}^{2}=E_{2}^{-1}, \quad E_{2}^{2}=E_{1}, \quad E_{1}^{2}=1 \\
E_{5}^{2}=E_{1}, \quad E_{4}^{2}=E_{3}^{-1}, & E_{3}^{2}=E_{2}^{-1}, \quad E_{2}^{2}=E_{1}, \quad E_{1}^{2}=1 \\
E_{5}^{2}=1, \quad E_{1}^{2}=E_{3}^{-1} E_{1}, & E_{3}^{2}=E_{0}^{-1}, \quad E_{2}^{2}=E_{1}, \quad E_{1}^{2}=1
\end{array}
$$

Ho già osservato che i gruppi $G_{i}^{3}$, che posseggono un divisore Abeliano d'indice $p$, sono necessariamente gruppi $G_{5,3}^{3,2}$; ma è ancora notevole il fatto che i rimanenti $p$ divisori d'indice $p$ sono gruppi $G_{4}^{2,2}$ : ciò risulta dalle formole di composizione trovate nei tre ultimi numeri.

43. Si abbia ora un gruppo $G_{3^{3,2}}^{3}$ tale che in esso non sia contenuto alcuno divisore Abeliano d'indice $p$.

Sia $G_{3}$ un divisore normale di $G_{3}^{3,2}$ appartenente al gruppo $K$ ed $E_{2}$ un elemento di $G_{2}$ fuori di $H$ : ragionando come al $n^{0} 13$ del $\S$ II, si dimostra che esiste un elemento $E_{3}$, fuori di $G_{2}$, che è invertibile con $E_{2}$; poi, considerando il gruppo Abeliano $G_{3}$ generato dall'elemento $E_{3}^{r}$ e dagli elementi 
di $G_{2}$, si dimostra ancora che esiste un elemento $E_{4}$ fuori di $G_{3}$, che è pure invertibile con $E_{2}$. Il gruppo $G_{4}$ generato dall'elemento $E_{4}$ e dagli elementi di $G_{3}$ è un $G_{4}{ }^{1}$ che ha come divisore invertibile in gruppo $G_{2}$. Questo gruppo $G_{1}^{1}$ deve contenere il gruppo $K$ di $G_{5}{ }^{3,2}$ ed io pongo $K=G_{3}$; poi, denotando con $E_{1}$ un elemento generatore del gruppo $H=G_{1}$, considero la serie di composizione:

$$
G_{5}^{3,2}, G_{4}^{1}, G_{3}, G_{2}, G_{1} .
$$

Dopo ciò, ponendo :

$$
E_{4}^{-1} E_{3} E_{4}=E_{3} E_{2}^{\prime \prime}, \quad E_{5}^{-1} E_{2} E_{5}^{\prime}=E_{2} E_{1}^{\prime},
$$

l'elemento $E_{2}^{\prime \prime}$ non è l'elemento identico perchè il gruppo $G_{4}^{1}$ non è Abeliano; inoltre, giacchè $E_{3}^{p}$ sta in $G_{2}$, risulta $E_{2}^{\prime \prime p}=1$. Ora, il gruppo ciclico generato dalle potenze di $E_{2}{ }^{\prime \prime}$ è l'unico della sua specie contenuto in $G_{4}{ }^{1}$, e siccome $G_{4}{ }^{1}$ è divisore normale di $G_{5}^{3,2}$, il detto gruppo è anche divisore normale di $G_{5}^{3,2}$ e coincide quindi con $G_{1}$ : si può dunque ritenere $E_{2}^{\prime \prime}=E_{1}$.

Similmente, $E_{1}^{\prime}$ non è l'elemento identico altrimenti l'elemento $E_{2}$, che è fuori di $H$, risulterebbe permutabile anche con $E_{5}$ e quindi con ogni elemento di $G_{5}^{3,2}$ : dunque si ha $E_{1}^{\prime}=E_{1}^{h}$ essendo l'intero $h$ primo con $p$.

Si hanno perciò le formole:

$$
\left.\begin{array}{lll}
E_{5}^{-1} E_{4} E_{5}=E_{4} E_{3}{ }^{\prime}, & E_{5}^{-1} E_{3} E_{5}=E_{3} E_{2}{ }^{\prime}, & E_{5}^{-1} E_{2} E_{5}=E_{2} E_{1}^{h},
\end{array}\right)
$$

Io comincio coll'osservare che il gruppo $K$ non può essere ciclico.

Infatti, giacchè il gruppo $G_{5}^{3,2}$ non contiene per ipotesi alcun divisore Abeliano d'indice $p$, la potenza $E_{5}^{\prime \prime}$ non è di grado superiore a $p^{2}$ e quindi sta in $G_{2}$. Allora l'elemento $E_{3}^{\prime}$ che figura nelle $(\tilde{E})$ non sta in $G_{2}^{\prime}$ perchè, in tale caso, i quattro elementi $E_{5}^{\prime}, E_{4}^{\prime}, E_{2}, E_{1}^{\prime}$ generano un gruppo di grado $p^{4}$ e questo gruppo non conterrebbe $K$. Ponendo dunque:

dalle (5) risulta :

$$
E_{3}^{\prime}=E_{3}^{\prime}, \quad E_{3}^{p}=E_{2}^{\prime},
$$

$$
E_{5}^{-1} E_{4}^{p} E_{5}=E_{4}^{p} E_{3}^{p} E_{1}^{\left(\begin{array}{l}
p \\
2
\end{array}\right)}=E_{4}^{p} E_{2} E_{1}^{\left(\begin{array}{l}
p \\
2
\end{array}\right)}
$$

ciò è assurdo giacchè, dovendo la potenza $E_{4}^{n}$ appartenere a $C_{n}$, si ha:

$$
E_{5}^{-1} E_{4}^{p} E_{5} \equiv E_{4}^{p}, \quad\left(\bmod G_{1}\right) \text {. }
$$


44. Si ammetta, in prima ipotesi, che il gruppo Abeliano $K$ abbia tutti $\mathrm{i}$ suoi elementi di grado $p$; in altri termini, che sia $G_{3}$ un gruppo $G_{3}{ }^{0,3}$.

Supponendo allora $p>3$, dalle (5) risulta:

$$
\begin{aligned}
& E_{5}^{-p} E_{4} E_{5}^{p}=E_{4}, \quad E_{5}^{-p} E_{3} E_{5}^{p}=E_{3}, \quad E_{5}^{-p} E_{2} E_{5}^{p}=E_{8}, \\
& E_{5}^{-1} E_{4}^{p} E_{5}=E_{4}^{p}, \quad E_{4}^{-p} E_{3} E_{4}^{p}=E_{3}, \quad E_{4}^{-p} E_{2} E_{4}^{p}=E_{2} ;
\end{aligned}
$$

quindi gli elementi $E_{5}^{p}, E_{4}^{p}$, i quali riescono invertibili con qualsiasi elemento di $G_{5}^{3,2}$, appartengono ad $H$.

Ciò posto, se $E_{3}{ }^{\prime}$ potesse appartenere a $G_{2}$, i quattro elementi $E_{5}, E_{4}$, $E_{2}, E_{1}$ genererebbero un gruppo di grado $p^{4}$ il quale non conterrebbe $K$ : dunque, si può porre $E_{3}^{\prime}=E_{3}$. Similmente, se $E_{2}{ }^{\prime}$ potesse appartenere a $G_{1}$, i quattro elementi $E_{5}^{4}, E_{4}, E_{3}, E_{4}$ genererebbero un gruppo di grado $p^{4}$ il quale non conterrebbe $K$ : dunque si puo porre $E_{2}{ }^{\prime}=E_{2}$.

Dopo quello che si è detto, si possono scrivere le formole:

$$
\begin{gathered}
E_{5}^{-1} E_{4} E_{5}=E_{1} E_{3}, \quad E_{5}^{1} E_{3} E_{5}=E_{3} E_{2}, \quad E_{5}^{-1} E_{2} E_{5}=E_{2} E_{1}^{h}, \\
E_{4}^{-1} E_{3} E_{4}=E_{3} E_{1}, \quad E_{4}^{-1} E_{2} E_{4}=E_{2}, \quad E_{3}^{-1} E_{2} E_{3}=E_{2} . \\
E_{5}^{p}=E_{1}^{\prime,}, \quad E_{4}^{n}=E_{1}^{3}, \quad E_{3}^{p}=1, \quad E_{2}^{p}=1, \quad E_{1}^{p}=1 .
\end{gathered}
$$

Col solito ragionamento si prova che queste formole definiscono sempre un gruppo $G_{5}^{3,2}$, purchè si pensi che $E_{4}$ appartiene ad $H$ e che è $p>3$.

Inoltre si osservi che si può ritenere $h=1$, perchè, se fosse altrimenti, eseguendo la trasformazione:

$$
\left(\begin{array}{ccccc}
E_{5}, & E_{4}, & E_{3}, & E_{2}, & E_{1} \\
E_{5}, & E_{4}^{h}, & E_{3}^{h} E_{1}^{(h)}, & E_{2}^{h}, & E_{1}^{h}
\end{array}\right),
$$

la quale non altera la forma delle (6) e (7), si porta subito il numero $h$ nel resto 1 di $p$.

Se:

$$
\mathbf{E}_{5}, \quad \mathbf{E}_{4}, \quad \mathbf{E}_{3}, \quad \mathbf{E}_{2}, \quad \mathbf{E}_{1},
$$

è una seconda base canonica definita come la prima, si vede immediatamente sulle formole $(6)$ e $(7)$ che le trasformazioni definite dalle congruenze:

$$
\mathbf{E}_{5} \equiv E_{5}, \quad \mathbf{E}_{4} \equiv E_{4}, \quad\left(\bmod G_{3}\right)
$$

non alterano gl' interi $\alpha, \beta, h$; dunque io posso considerare come un' unica trasformazione tutte quelle definite da:

$$
\mathbf{E}_{5} \equiv E_{5}^{u} E_{4}^{v}, \quad \mathbf{E}_{4} \equiv E_{4}^{s}, \quad\left(\bmod G_{3}\right),
$$


per dati valori degli interi $u, v, s$, i quali d'altronde debbono essere tali che il prodotto $u s$ risulti primo con $p$.

Gli elementi $\mathbf{E}_{3}, \mathbf{E}_{2}, \mathbf{E}_{1}$, che bisogna associare agli elementi $\mathbf{E}_{3}, \mathbf{E}_{4}$ ultimamente definiti, sono tali che:

$$
\mathbf{E}_{3} \equiv E_{3}^{r \varepsilon s}\left(\bmod G_{2}\right), \quad \mathbf{F}_{2} \equiv E_{2}^{u^{*} s}\left(\bmod G_{1}\right), \quad \mathbf{E}_{1}=E_{1}^{u s^{2}}
$$

quindi, dopo avere osservato che è :

$$
\left(E_{5} E_{4}\right)^{n}=E_{5}^{n} E_{4}^{n} E_{3}^{\left(\begin{array}{l}
n \\
2
\end{array}\right)} E_{2}^{\left(\begin{array}{l}
n \\
3
\end{array}\right)} F_{2}^{\left(\begin{array}{l}
n \\
4
\end{array}\right)}
$$

e perciò, avendo supposto $p>3$, che è :

$$
\left(E_{5} E_{4}^{\prime}\right)^{\prime \prime}=E_{5}^{\prime \prime} E_{4}^{p}
$$

si vede facilmente che la detta trasformazione fa subire agli 'interi $\alpha, \beta, h$ la sostituzione congrua :

$$
s^{2} u \alpha_{1} \equiv \alpha u+\beta v, \quad s u \beta_{1} \equiv \beta, \quad s h_{1} \equiv u^{2} h, \quad(\bmod p) .
$$

Avendo supposto $h=1$, se si vuole che sia ancora $h_{1}=1$, bisogna prendere $s=u^{2}(\bmod \eta)$ e quindi si ha:

$$
u^{5} \alpha_{1} \equiv u \alpha+v \beta, \quad u^{3} \beta_{1} \equiv \beta, \quad(\bmod p) \text {. }
$$

Osservo anzitutto che ogni volta che non è $\beta$ un multiplo di $p$, si può determinare il numero $v$ in modo che sia $\alpha_{1}=0(\bmod p)$.

Ciò posto, io distinguo i seguenti quattro casi possibili :

$$
p \equiv-1, p \equiv-5, p \equiv 5, \quad p \equiv 1, \quad(\bmod 12) \text {. }
$$

Nel primo caso, il numero $p-1$ non è divisibile nè per 3 nè per 4 e quindi ogni numero è cubo $(\bmod p)$ ed ogni numero quadrato (mod $p)$ ¿̀ un biquadrato $(\bmod p)$.

Dunque, se $\beta$ non è multiplo di $p$, si può determinare $u$ in modo che $\beta_{1}$ sia il resto 1 di $p$ : se invece è $\beta$ un multiplo di $p$, tutte le volte che non è $\alpha$ un multiplo di $p$, si può determinare $u$ in modo che sia $0 \alpha_{1} \equiv 1(\bmod p)$ ovvero $\alpha_{1} \equiv \varepsilon(\bmod p)$, secondo che $\alpha$ è della forma $u^{4}$ ovvero della forma $\varepsilon u^{4}$ rispetto a mod $p$.

Ottengo quindi quattro gruppi $G_{5}^{3,2}$, che corrispondono alle seguenti modificazioni delle (7): 


\begin{tabular}{|lllll}
$E_{5}^{p}=1$, & $E_{4}^{p}=E_{1}$, & $E_{3}^{p}=1$, & $E_{2}^{p}=1$, & $E_{1}^{p}=1$ \\
\hline$E_{5}^{p}=1$, & $E_{4}^{p}=1$, & $E_{3}^{p}=1$, & $E_{2}^{p}=1$, & $E_{1}^{p}=1$ \\
\hline$E_{5}^{p}=E_{1}$, & $E_{4}^{p}=1$, & $E_{3}^{p}=1$, & $E_{2}^{p}=1$, & $E_{1}^{p}=1$ \\
\hline$E_{5}^{p}=E_{1}^{\varepsilon}$, & $E_{4}^{p}=1$, & $E_{3}^{p}=1$, & $E_{2}^{p}=1$, & $E_{1}^{p}=1$ \\
\hline
\end{tabular}

Nel secondo caso, $p-1$ è divisibile per 3 ma non per 4, quindi ogni numero ha, rispetto a $\bmod p$, una delle forme:

$$
u^{3}, \quad \varepsilon u^{3}, \quad \varepsilon^{2} u^{3} ;
$$

allora, se $\beta$ è della prima forma, si hanno i quattro gruppi trovati precedentemente, ma se $\beta$ è della seconda o della terza forma, si hanno due nuovi gruppi $G_{5}^{3,2}$, i quali corrispondono alle seguenti modificazioni delle (7):

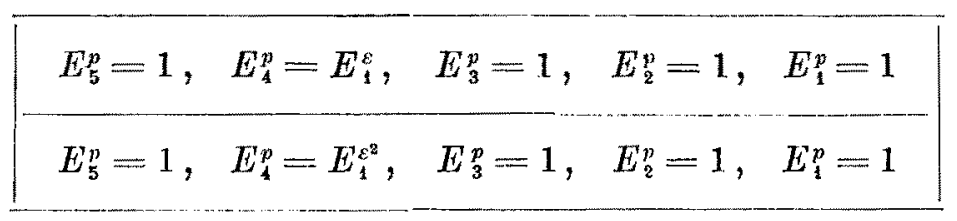

Nel terzo caso, $p-1$ è divisibile per 4 ma non per 3 , quindi ogni numero ha, rispetto a $\bmod p$, una delle forme:

$$
u^{4}, \quad \varepsilon u^{4}, \quad \varepsilon^{2} u^{4}, \quad \varepsilon^{3} u^{4} .
$$

I due ultimi gruppi trovati sono ora isomorfi al primo dei quattro gruppi che si riferiscono al primo caso; ma, oltre a questi, si hanno ancora due nuovi gruppi i quali si presentano quando è $\beta$ un multiplo di $p$ e che corrispondono alle seguenti modificazioni delle formole (7):

$$
\begin{array}{ll}
E_{5}^{p}=E_{1}^{\varepsilon^{2}}, & E_{4}^{p}=1, \quad E_{3}^{p}=1, \quad E_{2}^{p}=1, \quad E_{1}^{p}=1 \\
E_{5}^{p}=E_{1}^{\varepsilon^{2}}, & E_{4}^{p}=1, \quad E_{3}^{p}=1, \quad E_{2}^{p}=1, \quad E_{1}^{p}=1
\end{array}
$$

Se si fosse nel secondo caso, questi due gruppi sarebbero isomorf rispettivamente ai due ultimi gruppi che si riferiscono al primo caso. 
Finalmente, nel quarto caso, $p-1$ è divisibile per 3 e per 4 e quindi gli otto gruppi scritti nei tre casi precedenti sono effettivamente distinti e sono tutti i possibili gruppi $G_{\overline{5}}^{3,2}$ che rientrano nell'attuale caso.

45. Nell'ultimo numero ho supposto che il gruppo $G_{5}{ }^{3,2}$ non abbia alcun divisore Abeliano d'indice $p$ e che il gruppo $K$, intersezione dei $p+1$ divisori d'indice $p$ di $G_{3^{3}, 2}$ abbia tutti $\mathrm{i}$ suoi elementi di grado $p$.

In queste ipotesi, io posso dimostrare che non esistono gruppi $G_{5}{ }^{3,2}$ di grado 243. Infatti, se è $p=3$, riferendomi alle formole (5), concludo come prima $E_{3}^{\prime}=E_{3}, E_{2}{ }^{\prime}=E_{2}$, e quindi dovrebbero sussistere le formole (6). Allora da queste si ricava:

$$
E_{5}^{-3} E_{4} E_{5}^{3}=E_{4} E_{3}^{3} E_{2}^{3} E_{1}^{h}=E_{4} E_{1}^{h}
$$

perciò l'elemento $E_{5}^{3}$ è un elemento di $K$ del tipo $E_{3}^{-h} E_{2}^{r k} E_{1}^{\beta}$. Questo elemento, essendo una potenza di $F_{5}$, dovrebbe essere invertibile con $E_{5}$ e ciò contraddice alle stesse formole (6).

Invece, se è $p=2$, esiste un gruppo di grado 32 ed uno solo, che soddisfa a tutte le attuali ipotesi. Allora l'elemento $E_{3}^{\prime}$ non può appartenere a $G_{1}$, perchè, se così fosse, il quadrato $E_{5}^{2}$, che appartiene a $G_{3}$, risulterebbe permutabile con $E_{4}$ e quindi dovrebbe essere contenuto in $G_{2}$, anzi in $G_{1}$, perchè il detto quadrato è invertibile con $E_{5}$ : ciò non può essere a meno che non sia $E_{2}^{\prime}$ un elemento di $G_{1} ;$ ma, in tale caso, il gruppo definito dalle $(5)$ e (7) sarebbe evidentemente un gruppo $G_{\mathrm{s}}^{3,5}$.

Si ammetta dunque in primo loogo $E_{3}^{\prime}=E_{2}$. Allora l'elemento $E_{5}^{2}$ non is invertibile con $E_{4}$ e quindi il detto elemento appartiene a $G_{3}$ ma non a $G_{2}$ : dunque posso ritenere $E_{5}^{\prime 2}=E_{3}$ e conseguentemente $L_{2}^{\prime \prime}=1$. Inoltre, l'elemento $E_{4}^{2}$ risulta invertibile con $L_{5}^{\prime}$ e perciò sta in $G_{1}$; anzi io ritengo addirittura $E_{4}^{2}=1$, perchè, se fosse altrimenti, chiamerei $E_{4}$ l'elemento $E_{4} E_{3}$.

Dopo ciò posso scrivere le formole:

$$
\begin{aligned}
& E_{5}^{-1} E_{4} E_{5}=E_{1} E_{2}, \quad E_{5}^{-1} E_{3} E_{5}=E_{2}, \quad E_{5}^{-1} E_{2} E_{5}=E_{2} E_{4}, \\
& E_{4}^{-1} E_{3} E_{4}=E_{3} E_{1}, \quad E_{4}^{-1} E_{2} E_{4}=E_{2}^{2}, \quad E_{3}^{-1} E_{2} E_{3}=E_{2}, \\
& E_{5}^{2}=E_{3}, \quad E_{4}^{2}=1, \quad E_{3}^{2}=1, \quad E_{2}^{2}=1, \quad E_{1}^{2}=1,
\end{aligned}
$$

le quali definiscono effettivamente un gruppo $G_{5}^{3,2}$ di grado 32 .

Questo gruppo ha tre divisori di grado 16, ciascunn dei quali è generato da uno dei tre elementi:

$$
E_{4}, \quad E_{5}, \quad E_{5} E_{4}
$$

e dagli elementi di $G_{3}=K$. 
Mi resta ad esaminare il caso di $E_{3}^{\prime}=E_{3}$ : anche a questo caso corrisponde un gruppo di grado 32 , che soddisfa alle attuali ipotesi; ma questo gruppo, è isomorfo al precedente e si ottiene scambiando nelle ultime formole $E_{5}$ con $E_{4}$ e, nello stesso tempo, $E_{3}$ con $E_{2}^{*}$.

$\grave{\mathrm{E}}$ bene osservare che gli otto gruppi trovati nel numero precedente hanno un solo divisore $G_{4}^{1}$ e tutti gli altri $p$ divisori d'indice $p$ sono gruppi $G_{4}^{*}$; mentre, per l'unico gruppo di grado 32 ora trovato, accade ohe tutti i suoi divisori d'indice 2 sono gruppi $G_{4}^{1}$.

46. Si ammetta, in seconda ipotesi, che il gruppo $K$ sia un $G_{3}{ }^{0,2}$.

Allora il gruppo $K$ possiede un solo divisore d'indice $p$ tale che tutti i suoi elementi sono di grado $p$. Questo divisore è dunque normale in $G_{5}^{3,3} 0$ si può assumere come gruppo $G_{2}$.

Se $E_{2}$ è un elemento di $G_{2}$ funri di $G_{1}$, io mi costruisco come al $\stackrel{n}{n}$. 43 il gruppo $C_{4}^{1}$ costituito da tutti gli elementi di $C_{x_{5}^{3}}^{3,2}$ che sono permutabili con $E_{2}$ e poi considero la serie canonica:

ed una base :

$$
G_{5}^{3,2}, G_{4}^{1}, G_{3}^{0,2}, G_{2}, G_{1},
$$

$$
E_{3}, E_{4}, E_{3}, E_{2}, E_{1}
$$

relativa alla detta serie.

Dopo ciò, ragionando come nel n. 44 , concludo che, se è $p>2$, l'eltmento $E_{3}^{\prime}$ che figura nelle (5) non può appartenere a $G_{2}$ e quindi posso porre $E_{3}^{\prime}=E_{3}^{\prime}$.

L'elemento $E_{3}^{\prime}$ è nelle presenti ipotesi di grado $p^{2}$ e perciò la relazione:

$$
E_{5}^{-1} E_{4}^{p} E_{5}=E_{4}^{p} E_{3}^{p},
$$

mostra che la potenza $E_{4}^{p}$ non è invertibile con $E_{5}$ : dunque questa potenza è un elemento di $G_{2}$ fuori di $G_{H_{1}}$ e posso allora ritenere $E_{4}^{p}=E_{2}$. In tale caso la detta relazione si scrive:

$$
E_{5}^{-1} E_{2} E_{5}=E_{2} E_{3}^{p}
$$

e quindi deve necessariamente essere $E_{3}^{n}=E_{1}^{h}$.

Supponendo ora $p>3$, le formole (5) mostrano che la potenza $L_{5}^{n}$ non è invertibile con $E_{4}$ e quindi la detta potenza sta in $G_{3}$ ma non in $G_{2}$. Dunque il gruppo $G_{3}$ contiene elementi fuori di $G_{2}$ che sono invertibili con $E_{5}$ e per questo è necessario che l'elemento $k_{z}^{\prime}$ che figura nelle formole (5) appartenga a $G_{1}$. 
Le considerazioni precedenti portano alle formole:

$$
\left.\begin{array}{cl}
E_{5}^{-1} E_{4} E_{5}=E_{4} E_{3}, \quad E_{5}^{-1} E_{3} E_{5}=E_{3} E_{4}^{k}, \quad E_{5}^{-1} E_{2} E_{5}=E_{2} E_{1}^{h}, \\
E_{4}^{-1} E_{3} E_{4}=E_{3} E_{1}, \quad E_{4}^{-1} E_{2} E_{4}=E_{2}, \quad E_{3}^{-1} E_{2} E_{3}=E_{2},
\end{array}\right\}
$$

le quali, come subito dimostrerò, non possono definire un gruppo $G_{5}^{3,2}$ se non quando è:

$$
\alpha+h \equiv 0, \quad \beta \equiv k, \quad(\bmod p) .
$$

Infatti, trasformando la prima delle relazioni (9) mediante l'elemento $E_{4}$, si ha:

donde si ricava:

$$
E_{4}^{-1} E_{5}^{p} E_{4}=E_{3}^{\alpha} E_{1}^{\alpha} \cdot E_{2}^{\beta} E_{1}^{\gamma}=E_{5}^{p} E_{1}^{\alpha},
$$

$$
E_{5}^{-p} E_{4} E_{5}^{p}=E_{4} E_{1}^{-\alpha} \text {. }
$$

Ma dalla prima delle (s) e dalle altre si ha:

dunque :

$$
E_{5}^{-p} E_{4} E_{5}^{n}=E_{4} E_{3}^{p}=E_{4} E_{1}^{h} ;
$$

$$
\alpha+h \equiv 0, \quad(\bmod p) .
$$

Inoltre, l'elemento $E_{5}^{n}$ è invertibile con $E_{5}$ e quindi :

sicchè :

$$
E_{5}^{p}=E_{5}^{-1} E_{3}^{n} E_{2}^{\beta} E_{1}^{\gamma} E_{5}=E_{5}^{p} E_{1}^{q z k} E_{1}^{\beta h},
$$

$$
\alpha h+\beta h \equiv 0, \quad(\bmod p) ;
$$

poi, da questa congruenza e dalla precedente segue:

$$
\beta-k \equiv 0, \quad(\bmod p) .
$$

Ciò posto, eseguendo la trasformazione:

$$
\left(\begin{array}{ccccc}
E_{5}, & E_{4}, & E_{3}, & E_{2}, & E_{4} \\
E_{5}, & E_{4}^{h}, & E_{3}^{h} E_{1}^{\left(\frac{h}{2}\right)^{k}}, & E_{2}^{h}, & E_{1}^{h^{2}}
\end{array}\right),
$$

l'intero $h$ nel resto 1 di $p$; ed eseguendo la trasformazione:

$$
\left(\begin{array}{ccccc}
E_{5}, & E_{4}, & E_{3}, & E_{2}, & E_{1} \\
E_{5} E_{4}, & E_{4}, & E_{3} E_{1}, & E_{2}, & E_{4}
\end{array}\right),
$$

i numeri $k$ e $\beta$ si cambiano in $k+1$ e $\beta+1$. Finalmente, eseguendo la tras- 
formazione :

$$
\left(\begin{array}{ccccc}
E_{5}, & E_{4}, & E_{3}, & E_{2}, & E_{1} \\
E_{5} E_{3}, & E_{4}, & E_{3} E_{1}^{-1}, & E_{2}, & E_{1}
\end{array}\right)
$$

si cambia soltanto $\gamma$ in $\gamma+h$.

Supponendo dunque $h \equiv 1, k \equiv 0, \gamma \equiv 0(\bmod p)$, le $(10)$ dànno $\alpha \equiv-1$, $\beta \equiv 0(\bmod p)$, e quindi non può, nella presente ipotesi, esistere che un solo gruppo $G_{5}^{3,2}$ corrispondente alle formole:

$$
\left.\begin{array}{l}
E_{5}^{-1} E_{4} E_{5}=E_{4} E_{3}, \quad E_{5}^{-1} E_{3} E_{5}=E_{3}, \quad E_{5}^{-1} E_{2} E_{5}=E_{2} E_{1} \\
E_{4}^{-1} E_{3} E_{4}=E_{3} E_{1}, \quad E_{4}^{-1} E_{2} E_{4}=E_{2}, \quad E_{3}^{-1} E_{2} E_{3}=E_{2}, \\
E_{5}^{p}=E_{1}^{-1}, \quad E_{4}^{p}=E_{2}, \quad E_{3}^{p}=E_{1}, \quad E_{2}^{p}=1, \quad E_{3}^{p}=1 .
\end{array}\right)
$$

D'altra parte è facile verificare che queste formole, se è $p>3$, definiscono effettivamente un gruppo $G_{5}{ }^{3,2}$ tale che il relativo gruppo $K$ è un $G_{3}{ }^{0,2}$.

I $p+1$ divisori d'indice $p$ del gruppo ora trovato sono gruppi $G_{1}^{4}$ : uno solo di essi ha come divisore invertibile il gruppo $G_{2}$ e gli altri hanno il divisore invertibile ciclico.

47. Caso di $p=3$.

Riferendomi sempre alle formole (5) si conclude, come nel numero precedente, $E_{3}^{\prime}=E_{3}, E_{4}^{3}=E_{2} ;$ poi, ammettendo l'jpotesi che $E_{2}^{\prime}$ appartenga a $G_{1}$, con un ragionamento identico a quello fatto nel detto numero, si trova un gruppo $G_{5}{ }^{3,2}$ di grado 243 , che è definito dalle formole (11) quando vi si suppone $p=3$.

Ma oltre a questo gruppo, ne esiste un altro di grado 243 , che si presenta quando si fa l'ipotesi che l'elemento $E_{2}^{\prime}$ non appartenga a $G_{1}$. In tale ipotesi io definisco l'elemento $E_{2}$ mediante la relazione:

$$
E_{5}^{-1} E_{3} E_{5}=E_{3} E_{2}
$$

ed osservo che allora il gruppo $G_{3}$ non contiene alcun elemento permutabile con $E_{5}$, fatta eccezione degli elementi di $G_{1}$ : dunque la potenza $E_{5}^{3}$ appartiene a $G_{1}$.

Cjò posto, la relazione:

$$
E_{5}^{-3} E_{4} E_{5}^{3}=E_{4} E_{3}^{3} E_{2}^{3} E_{1}^{h}
$$

dà $L_{3}^{3}=L_{1}^{2 h} ;$ poi la relazione:

$$
E_{5}^{-1} E_{4}^{3} E_{5}^{\prime}=E_{4}^{3} E_{3}^{3}=E_{4}^{3} E_{1}^{2 h},
$$

mostra che la potenza $E_{1}^{p}$ è un elemento di $G_{2}$ del tipo $E_{2}^{2} E_{1}^{\beta}$. 
Dietro le considerazioni che precedono hanno luogo le formole:

$$
\begin{aligned}
& E_{5}^{-1} E_{4} E_{5}=E_{4} E_{3}, \quad E_{5}^{-1} E_{3} E_{5}=E_{3} E_{2}, \quad E_{5}^{-1} E_{2} E_{5}=E_{2} E_{1}^{h}, \\
& E_{4}^{-1} E_{3} E_{4}=E_{3} E_{1}, \quad E_{4}^{-1} E_{2} E_{4}=E_{2}, \quad E_{3}^{-1} E_{2} E_{3}=E_{2}, \\
& E_{5}^{3}=E_{1}^{n}, \quad E_{4}^{3}=E_{2}^{2} E_{1}^{\beta}, \quad E_{3}^{3}=E_{1}^{2 h}, \quad E_{2}^{3}=1, \quad E_{1}^{3}=1 .
\end{aligned}
$$

Eseguendo la trasformazione:

$$
\left(\begin{array}{ccccc}
E_{5}, & E_{1}, & E_{3}, & E_{z}, & E_{1} \\
E_{5}, & E_{4}^{2}, & E_{3}^{2} E_{1}^{2}, & E_{2}^{2}, & E_{1}
\end{array}\right),
$$

il numero $h$ si cambia in $2 h$ e quindi posso ritenere che sia $h=1$.

Allora, chiamando $E_{5}^{\prime}, E_{4}$ rispettivamente gli elementi $E_{5} E_{3}, E_{4} E_{3}$, gl'interi a e $\beta$ si possono fare aumentare, ciascuno in modo indipendente, di una unità.

Dunque, giacchè si può supporre $\alpha \equiv \beta \equiv 0(\bmod 3)$, si ha soltanto un nuovo gruppo $G_{5}{ }^{3,2}$ di grado 243 , che si può ritenere definito dalle ultime formole quando ivi si fa $h=1, \alpha=\beta=0$.

4s. Caso di $p=2$.

Ragionando come nel $n^{\circ} 45$, si conclude che l'elemento $E_{3}^{\prime}$ che figura nelle formole (5) non può appartenere a $G_{1}$.

Se si ammette in primo luogo $E_{3}^{\prime}=E_{2}$, il quadrato $E_{5}^{2}$ non risulta invertibile con $E_{4}$ e quindi esso è un elemento di $G_{3}$ fuori di $G_{2}$ : posso perciò supporre $E_{5}^{2}=E_{3}^{\prime}$ e conseguentemente $E_{2}^{\prime}=1$.

L'elemento $E_{4}$ risulta invertibile con $E_{5}$ e percio sta in $H$; inoltre l'elemento $E_{3}^{2}$, che è pure invertibile con $E_{5}$, sta in $H$ e, siccome $E_{3}$ è di grado 4 per ipotesi, risulta $E_{3}^{2}=E_{1}$.

Dunque, supponendo $E_{3}^{\prime}=E_{2}$, si hanno soltanto due possibili gruppi $G_{5}^{3,2}$ di grado 32 definiti dalle seguenti modificazioni delle (5):

$$
\begin{aligned}
& E_{5}^{-1} E_{4} E_{5}=E_{4} E_{2}, \quad E_{5}^{-1} E_{3} E_{5}=E_{3}, \quad E_{5}^{-1} E_{2} E_{5}=E_{2} E_{4}, \\
& E_{4}^{-1} E_{3} E_{4}=E_{3} E_{1}, \quad E_{4}^{-1} E_{2} E_{4}=E_{2}, \quad E_{3}^{-1} E_{2} E_{3}=E_{2},
\end{aligned}
$$

e dalle formole:

$$
\begin{aligned}
& E_{5}^{2}=E_{3}, \quad E_{4}^{2}=E_{1}, \quad E_{3}^{2}=E_{1}, \quad E_{2}^{2}=1, \quad E_{1}^{2}=1 \\
& E_{5}^{2}=E_{3}, \quad E_{4}^{2}=1, \quad E_{3}^{2}=E_{1}, \quad E_{2}^{2}=1, \quad E_{1}^{2}=1
\end{aligned}
$$


Si può subito verificare che questi due gruppi realmente esistono e non sono isomorfi, perchè i corrispondenti gruppi $G_{4}^{1}$, i quali si sono presentati nel n. 12 del $\S$ II, zon sono isomorfi.

Nelle attuali ipotesi, non accade il fatto notato al $n .^{\circ} 45$ cjoc, non esiste un jsomorfismo che porti l'elemento $E_{2}$ nell'elemento $E_{3}$; quindi io debbo continuare la mia analisi supponendo in secondo luogo $E_{3}{ }^{\prime}=E_{3}$.

Si osservi che gli elementi di grado 4 contenuti in $G_{3}$ hamno tutti in stesso quadrato e quindi $E_{3}^{2}$ è invertibile con $E_{5}$ e con ogni elemento del gruppo prineipale: dunque deve essere $E_{2}^{\prime}$ un elemento di $H$ ed $E_{3}^{\prime}=E_{1}$.

Io posso ritenere $E_{2}^{\prime}=1$ giacchi, se fosse altrimenti, chiamerei $E_{5}$ l'elemento $E_{5} E_{4}$; allora le $(5)$ forniscono le relazioni:

$$
E_{5}^{-1} E_{4}^{2} E_{5}=E_{4}^{2} E_{3}^{2} E_{1}, \quad E_{5}^{-2} E_{4} E_{5}^{2}=E_{4} E_{3}^{2},
$$

le quali mostrano che l'elomento $E_{4}^{2}$ è invertibile con $E_{5}$ e perciò esso appartiene ad $I I$, e che l'elemento $E_{5}^{2}$ non è invertibile con $E_{4}$ e perciò esso è un elemento di $G_{3}$ fuori di $G_{2}$.

Ponendo quindi:

$$
E_{3}^{2}=E_{3} E_{2}^{\beta} E_{1}^{\gamma},
$$

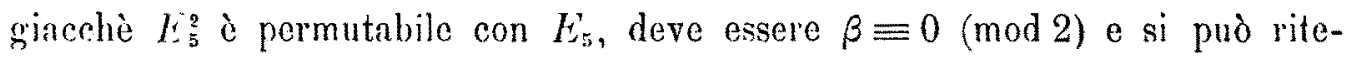
nere anche $\gamma \equiv 0(\bmod 2)$ perchè, in caso contrario, si chiamerebbe $E_{\mathrm{s}}$ l'elemento $E_{5} E_{3}$.

Dunque, quando è $E_{3}^{\prime}=E_{3}$, si hanno soltanto due possibili gruppi $C_{5}{ }^{32}$ di grado 32 definiti dalle seguenti modificazioni delle (5):

$$
\begin{array}{ll}
E_{5}^{-1} E_{4} E_{5}=E_{4} E_{3}, & E_{5}^{-1} E_{3} E_{5}=E_{3}, \quad E_{5}^{-1} E_{2} E_{5}=E_{2} E_{1}, \\
E_{4}^{-1} E_{3} E_{4}=E_{3} E_{1}, & E_{4}^{-1} E_{2} E_{4}=E_{2}, \quad E_{3}^{-1} E_{2} E_{3}^{\prime}=E_{2},
\end{array}
$$

e dalle formole (12).

In conclusione, esistono quattro, e non più, gruppi $G_{5}{ }^{3,2}$ di grado 32 tali che i corrispondenti gruppi $K$ sono gruppi $G_{3}{ }^{0,2}$.

49. Io riassumo brevemente i risultati ottenuti nel presente paragrafo.

Esistono soltanto otto gruppi $G_{5}^{3,2}$ di grado 32 e soltanto sei gruppi $G_{5}{ }^{3 \text { ? }}$ di grado 243.

Se poi è $p>3$ bisogna distinguere i seguenti quattro casi:

$$
p \equiv-1, p \equiv 5, \quad p \equiv-5, p \equiv 1,(\bmod 12) \text {. }
$$

Quando il numero primo $p$ soddisfa alla prima congruenza, esistono solamente otto gruppi $G_{5}^{3,2}$, quando $p$ soddisfa alla seconda congruenza esistono 
solamente dieci gruppi $G_{5}{ }^{3,2}$, quando $p$ soddisfa alla térza congruenza esistono solamente dodici gruppi $G_{5}{ }^{3,2}$; finalmente, quando il numero primo $p$ soddisfa alla quarta congruenza esistono soltanto quattordici grappi $G_{5}^{3,2}$.

Tutti i detti gruppi sono rappresentati nelle tre tabelle che seguono.

I.

Gruppi $G_{5}^{3,2}$ di grado 32 .

\begin{tabular}{lll}
$E_{5}^{-1} E_{4} E_{5}=E_{4} E_{3}, \quad E_{5}^{-1} E_{3} E_{5}=E_{3} E_{2}$, & $E_{5}^{-1} E_{2} E_{5}=E_{2} E_{1}$, \\
$E_{4}^{-1} E_{3} E_{4}=E_{3}, \quad E_{4}^{-1} E_{2} E_{4}=E_{2}$, & $E_{3}^{-1} E_{2} F_{3}=E_{2}$. \\
\hline$E_{5}^{2}=1, \quad E_{4}^{2}=E_{3}^{-1}, \quad E_{3}^{2}=E_{2}^{-1}$, & $E_{2}^{2}=E_{1}, \quad E_{1}^{2}=1$. \\
\hline$E_{5}^{2}=E_{1}, \quad E_{4}^{2}=E_{3}^{-1}$, & $E_{3}^{2}=E_{2}^{-1}$, & $E_{2}^{2}=E_{1}, \quad E_{1}^{2}=1$. \\
$E_{5}^{2}=1, \quad E_{4}^{2}=E_{3}^{-1} E_{1}$, & $E_{3}^{2}=E_{2}^{-1}$, & $E_{2}^{2}=E_{1}, \quad E_{1}^{2}=1$.
\end{tabular}

\begin{tabular}{|c|l|}
\hline$E_{5}^{-1} E_{4} E_{5}=E_{1} E_{2}, \quad E_{5}^{-1} E_{3} E_{5}=E_{3}$, & $E_{5}^{-1} E_{2} E_{5}=E_{2} E_{1}$, \\
$E_{4}^{-1} E_{3} E_{1}=E_{3} E_{1}, \quad E_{4}^{-1} E_{2} E_{4}=E_{2}$, & $E_{3}^{-1} E_{2} E_{3}=E_{2}$. \\
\hline$E_{5}^{2}=E_{3}, \quad E_{4}^{2}=1, \quad E_{3}^{2}=1, \quad E_{2}^{2}=1, \quad E_{1}^{2}=1$. \\
\hline$E_{5}^{2}=E_{3}, \quad E_{4}^{2}=E_{1}, \quad E_{3}^{2}=E_{1}, \quad E_{2}^{2}=1, \quad E_{1}^{2}=1$. \\
\hline$E_{5}^{2}=E_{3}, \quad E_{4}^{2}=1, \quad E_{3}^{2}=E_{1}, \quad E_{2}^{2}=1, \quad E_{1}^{2}=1$. \\
\hline
\end{tabular}

$E_{5}^{-1} E_{4} E_{5}=E_{4} E_{3}, \quad E_{5}^{-1} E_{3} E_{5}=E_{3}, \quad E_{5}^{-1} E_{2} E_{5}=E_{2} E_{1}$, $E_{4}^{-1} E_{3} E_{4}=E_{4} E_{1}, \quad E_{4}^{-1} E_{2} E_{4}=E_{2}, \quad E_{3}^{-1} E_{2} E_{3}=E_{2}$.

$E_{\overline{0}}^{q}=E_{3}, \quad E_{4}^{q}=E_{1}, \quad E_{3}^{q}=E_{1}, \quad E_{2}^{2}=1, \quad E_{1}^{2}=1$.

$E_{5}^{q}=E_{3}, \quad E_{4}^{q}=1, \quad E_{3}^{q}=E_{1}, \quad E_{2}^{q}=1, \quad E_{1}^{q}=1$. 
II.

\section{Gruppi $G_{5}^{3,2}$ di grado 243.}

\begin{tabular}{l}
$E_{5}^{-1} E_{4} E_{5}=E_{4} E_{3}, \quad E_{5}^{-1} E_{3} E_{5}=E_{3} E_{2}, \quad E_{5}^{-1} E_{2} E_{5}=E_{2} E_{1}$, \\
$E_{4}^{-1} E_{3} E_{4}=E_{3}, \quad E_{4}^{-1} E_{2} E_{4}=E_{2}, \quad E_{3}^{-1} E_{2} E_{3}=E_{2}$. \\
\hline$E_{5}^{3}=1, \quad E_{1}^{3}=E_{2}^{2}, \quad E_{3}^{3}=E_{1}^{9}, \quad E_{3}^{3}=1, \quad E_{1}^{3}=1$. \\
\hline$E_{5}^{3}=1, \quad E_{4}^{3}=E_{0}^{a} E_{1}, \quad E_{3}^{3}=E_{1}^{2}, \quad E_{9}^{3}=1, \quad E_{1}^{3}=1$. \\
$E_{5}^{3}=E_{1}, \quad E_{4}^{3}=E_{2}^{3} E_{1}, \quad E_{3}^{3}=E_{1}^{2}, \quad E_{2}^{3}=1, \quad E_{1}^{3}=1$. \\
$E_{5}^{3}=E_{1}^{3}, \quad E_{4}^{3}=E_{2}^{3} E_{1}, \quad E_{3}^{3}=E_{1}^{2}, \quad E_{9}^{3}=1, \quad E_{1}^{3}=1$.
\end{tabular}

\begin{tabular}{ll}
$E_{5}^{-1} E_{4} E_{5}=E_{1} E_{3}, \quad E_{5}^{-1} E_{3} E_{5}=E_{3}$, & $E_{5}^{-1} E_{2} E_{5}=I_{2} E_{1}$ \\
$E_{4}^{-1} E_{3} E_{4}=E_{3} E_{1}, \quad E_{4}^{-1} E_{2} E_{4}=E_{2}$, & $E_{3}^{-1} E_{2} E_{3}=E_{2}$. \\
\hline$E_{5}^{3}=E_{1}{ }^{1}, \quad E_{1}^{3}=E_{2}, \quad E_{3}^{3}=E_{1}, \quad F_{2}^{3}=1, \quad E_{1}^{3}=1$.
\end{tabular}

$$
\begin{gathered}
E_{5}^{-1} E_{4} E_{5}=E_{1} E_{3}, \quad E_{5}^{-1} L_{3} E_{5}=E_{3} E_{1}, \quad E_{5}^{-1} E_{2} E_{5}=E_{2} E_{1}, \\
E_{4}^{-1} L_{3} E_{4}=I_{3} E_{1}, \quad E_{4}^{-1} E_{2} E_{4}=E_{2}, \quad E_{3}^{-1} E_{2} E_{3}=E_{2} . \\
E_{5}^{3}=1, \quad E_{4}^{3}=E_{2}^{2}, \quad E_{3}^{3}=E_{1}^{2}, \quad E_{3}^{3}=1, \quad E_{1}^{3}=1 .
\end{gathered}
$$


III.

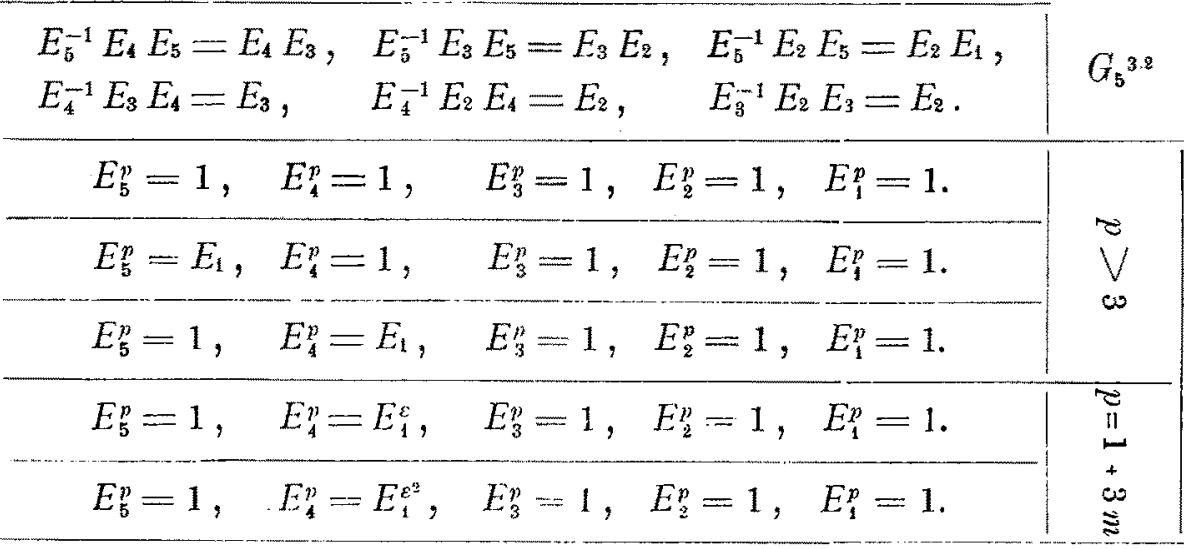

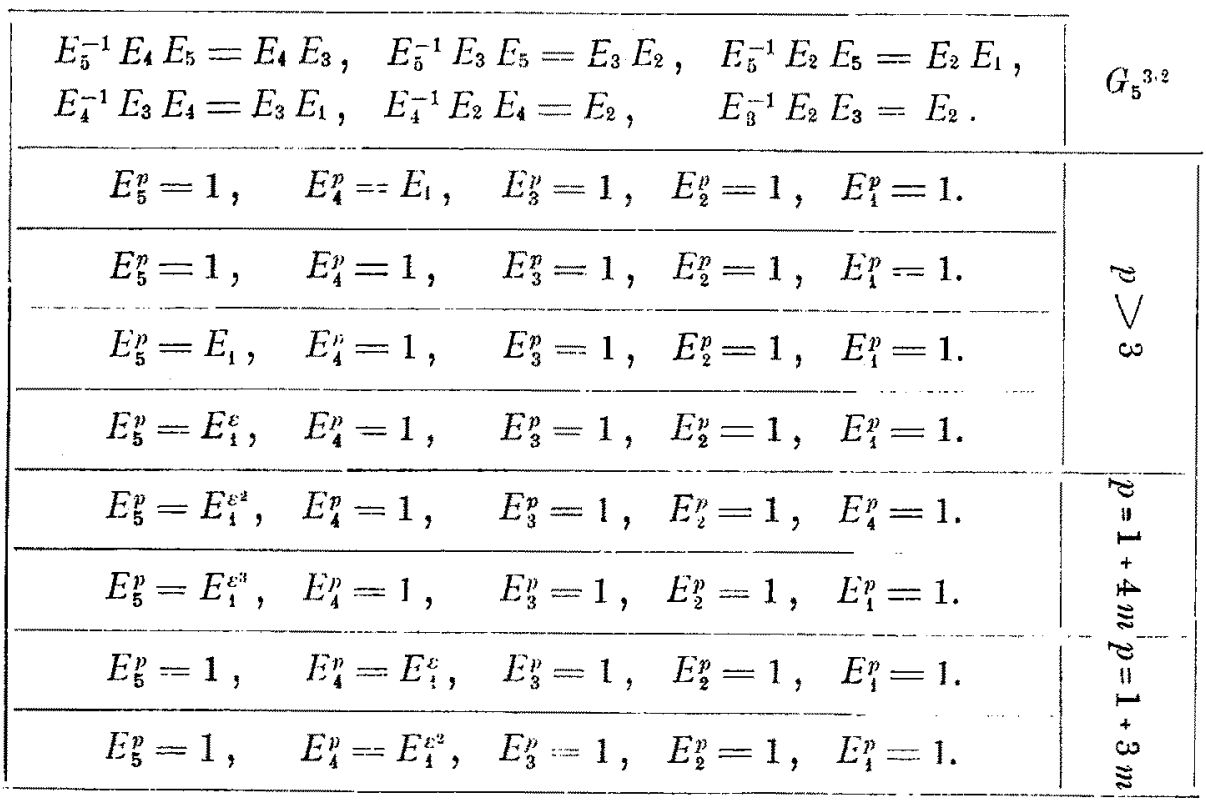

$$
\begin{array}{l|l}
E_{6}^{-1} E_{4} E_{5}=E_{4} E_{3}, \quad E_{5}^{-1} E_{3} E_{5}=E_{3}, \quad E_{5}^{-1} E_{2} E_{5}=E_{2} E_{1}, & G_{5}^{3.2} \\
E_{4}^{-1} E_{3} E_{4}=E_{3} E_{1}, \quad E_{4}^{-1} E_{2} E_{4}=E_{2}, \quad E_{3}^{-1} E_{2} E_{3}=E_{2} . & \\
E_{5}^{p}=E_{1}^{-1}, \quad E_{4}^{p}=E_{2}, \quad E_{3}^{p}=E_{1}, \quad E_{2}^{p}=1, \quad E_{1}^{p}=1 . & p>3
\end{array}
$$




\section{$\S$ VII. I Gruppi $G_{5}^{3,3}$ e $G_{5}^{34}$.}

50. Il gruppo $K$ intersezione di tutti i divisori d'indice $p$ di un gruppo $G_{\mathrm{s}}^{3,3}$ è di grado $p^{2}$. Sia $E_{1}$ un elemento generatore del divisore invertibile $H$ di $G_{5}{ }^{3,3}$ ed $E_{2}$ un elemento di $K$ fuori di $H$; indi si consideri il gruppo $G_{4}^{3}$ costituito da tutti gli elementi di $G_{5}^{3,3}$ che sono permutabili con $L_{2}$.

Denotando con $E_{5}$ un elemento di $G_{5}^{3,2}$ fuori di $G_{4}^{4}$, per definizione, non è $E_{5}^{i}$ permutabile con $E_{2}^{\prime}$; però, io voglio dimostrare ehe esistono elementi di $G_{4}^{3}$, fuori di $K$, che sono permutabili con $E_{5}$.

Siano $E_{4}^{\prime}$ ed $E_{3}$ due elementi di $G_{4}^{4}$ tali che $E_{4}$ non appartenga al gruppo di grado $y^{3}$ generato dall'elemento $k_{3}$ e dagli elementi di $K$. Ponendo:

$$
E_{5}^{-1} E_{4}^{\prime} E_{5}=E_{4} E_{2}^{w} E_{1}^{q}, \quad E_{5}^{-1} E_{3} E_{5}^{\prime}=E_{3} E_{2} E_{1}^{\gamma} \text {, }
$$

uno almeno degli interi $\alpha, \gamma$ deve essere primo con $p$. Infatti, se $\alpha$ e $\gamma$ sono due multipli di $p$, i tre elementi $E_{5}^{p}, E_{1}^{p}, L_{3}^{\prime}$ risultano permutabili con ogni clemento di $G_{5}^{3,3}$ e quindi debbono appartenere ad $H$; inoltre, per la ragione detta al n.. 43 si può sempre ritenere:

$$
L_{4}^{-1} E_{3} E_{4}^{\prime}=E_{3} E_{1}^{h}
$$

essendo $h$ primo con $p:$ quindi gli elementi $E_{5}, L_{4}, E_{3}, k_{1}$ generano un gruppo di grado $p^{4}$ e questo gruppo, giacchè non contiene l'elemento $E_{2}$, non contiene $K$.

Ciò posto, l'elemento $E_{4}^{\gamma} E_{3}^{-\alpha}$ è fuori di $K$ e si ha:

$$
E_{5}^{-1}\left(E_{4}^{\gamma \gamma} L_{3}^{-\alpha}\right) E_{5}^{\prime}=\left(E_{4}^{\gamma-\gamma} L_{3}^{\gamma \alpha}\right) E_{1}^{\alpha \alpha-\beta \gamma} \text {. }
$$

Ora, giacchè $E_{5}$ non è invertibile con $E_{2}$, ponendo:

$$
E_{5}^{-1} E_{2} E_{5}^{\prime}=E_{2}^{\prime} E_{1}^{\prime}
$$

risulta subito che l'elemento:

$$
E_{4}^{-\gamma} E_{3}^{\gamma} E_{2}^{-(x+\gamma-\beta \gamma)}
$$

il quale non appartiene a $K$, è permutabile con $E_{5}$.

Io assumo un tale elemento come clemento $E_{3}$ e, scelto poi $E_{4}$ fuori del gruppo $G_{3}$ generato da $E_{3}$ e dagli elementi di $K$, definisco l'elemento $E_{2}$ medianto la relezione:

$$
E_{5}^{-1} E_{4} E_{5}^{\prime}=E_{4} E_{2}
$$


inoltre, posso ritenere che sia:

$$
E_{4}^{-1} E_{3} E_{\text {t }}=E_{3} E_{1},
$$

perchè, in caso contrario, chiamerei $E_{3}$ una conveniente potenza di $E_{3}$. Ciò posto, gli elementi:

$$
E_{\mathrm{s}}, E_{4}, E_{3}, E_{2}, E_{1},
$$

definiti nella detta maniera costituiscono una base canonica di un gruppo $G_{5}^{3,3}$ e soddisfano alle relazioni :

$$
\begin{aligned}
& E_{5}^{-1} E_{4} E_{5}=E_{4} E_{2}, \quad E_{5}^{-1} E_{3} E_{5}=E_{3}, \quad E_{5}^{-1} E_{2} E_{5}=E_{2} E_{1}, \\
& E_{4}^{-1} E_{3} E_{4}=E_{3} E_{1}, \quad E_{4}^{-1} E_{2} E_{4}=E_{2}, \quad E_{3}^{-1} E_{2} E_{3}=E_{2} .
\end{aligned}
$$

51. L'elemento $E_{\overline{5}}^{p}$ appartiene a $K$ ed è permutabile con $E_{5}$, quindi deve essere $E_{5}^{p}$ un elemento di $H$. Intanto dalle (1) si ricava:

$$
E_{5}^{-p} E_{4} E_{5}^{p}=E_{4} E_{2}^{p} E_{1}^{(p)}
$$

dunque, supponendo $p>2$, si ottiene $E_{2}^{p}=1$.

Allora, le formole:

$$
E_{5}^{1} E_{4}^{p} E_{5}^{\prime}=E_{4}^{p} E_{2}^{p}, \quad E_{4}^{-p} E_{3}^{\prime} E_{4}^{p}=E_{3} E_{1}^{p}
$$

mostrano che la potenza $E_{4}^{\prime}$ è invertibile con ogni elemento del grupro principale e perciò la detta potenza sta in $H$. Similmente la potenza $E_{z}^{n}$ è invertibile con ogni elemento del gruppo principale e percio sta in $H$ : dunque si hanno le relazioni:

$$
E_{b}^{p}=E_{1}^{*}, \quad E_{4}^{p}=F_{1}^{*}, \quad E_{3}^{p}=E_{1}^{\gamma}, \quad E_{2}^{p}=1, \quad E_{1}^{p}=1
$$

le quali, insieme alle (1), definiscono sempre un gruppo $\dot{x}_{5}^{3,3}$ purchè si tenga presente che è $p>2$.

Essendo $s$ un intero primo con $p$, si vede subito che eseguendo la trasformazione :

$$
\left(\begin{array}{ccccc}
E_{5}^{\prime}, & E_{4}, & E_{3}, & E_{2}, & E_{1} \\
E_{5} E_{3}^{*}, & E_{1}^{*} E_{3}^{\mu}, & E_{3}, & E_{2}^{s s} E_{1}^{-* i}, & E_{1}^{s}
\end{array}\right)
$$

la quale non altera la forma delle (1) e (2), i numeri o, $\beta, \gamma$ subiscono la sostituzione:

$$
s \alpha_{1} \equiv \alpha+i \gamma, \quad s \beta_{1} \equiv s \beta+\mu \cdot \gamma, \quad s \gamma_{1} \equiv \gamma \quad(\bmod p)
$$

quindi, se $\gamma$ è primo con $p$, determinando convenientemente $s, \lambda, \mu$, si puì 
supporre:

$$
\alpha_{1}=\beta_{1}=0, \quad \gamma_{1}=1
$$

Esiste allora un solo gruppo $G_{5}^{3,3}$ definito dalle relazioni (1) e dalle (2) modificate come segue:

$$
E_{5}^{p}=1, \quad E_{4}^{p}=1, \quad E_{3}^{p}=E_{1}, \quad E_{2}^{p}=1, \quad E_{1}^{p}=1
$$

Se il numero y è un multiplo di $p$, bisogna distinguere il caso in cui è $p>3$ dal caso in cui ̀̀ $p=3$.

Nel primo caso, se $u$ è un intero primo con $p$, eseguendo la trasformazione:

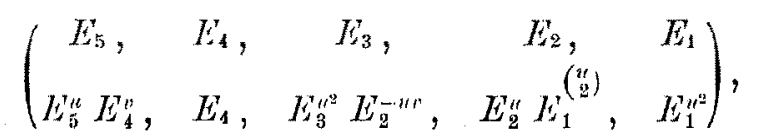

la quale non altera la forma delle (1) e (2), si vede facilmente che $\alpha$ e $\beta$ si portano rispettivamente in due numeri $\alpha_{1}$ e $\beta_{1}$ tali che:

$$
u^{2} \alpha_{1} \equiv u \alpha+v \beta, \quad u^{2} \beta_{1} \equiv \beta \quad(\bmod p) \text {. }
$$

Dunque, tulte le volte che è $\beta$ primo con $p$, si può determinare $u$ in $\operatorname{modo}$ che $\operatorname{sia} \beta_{1} \equiv 1(\bmod p)$ oppure $\beta_{1} \equiv \varepsilon(\bmod p)$; poi si determina $v$ in modo che sia $\alpha_{1} \equiv 0(\bmod p)$. Se invece è $\beta$ un multiplo di $p$, si può determinare $u$ in modo da portare $\alpha_{1}$ nel resto 1 ovvero nel resto 0 di $p$.

In conclusione, quando è $p>3$, oltre all'ultimo gruppo trovato esistono altri quattro, e non più, gruppi $G_{5}^{3,3}$ i quali sono definiti dalle (1) e dalle seguenti modificazioni delle formole (2):

$$
\begin{array}{ll}
E_{5}^{p}=1, \quad E_{4}^{p}=E_{1}, \quad E_{3}^{p}=1, \quad E_{2}^{p}=1, \quad E_{1}^{p}=1 \\
E_{5}^{p}=1, \quad E_{4}^{p}=E_{1}^{c}, \quad E_{3}^{p}=1, \quad E_{2}^{p}=1, \quad E_{1}^{p}=1 \\
\hline E_{5}^{p}=E_{1}, \quad E_{4}^{p}=1, \quad E_{3}^{p}=1, \quad E_{2}^{p=1}, \quad E_{1}^{p}=1 \\
\hline E_{5}^{p}=1, \quad E_{4}^{p}=1, \quad E_{3}^{p}=1, \quad E_{2}^{p}=1, \quad E_{1}^{p}=1
\end{array}
$$

I einque gruppi $G_{5}^{3,3}$ che ho trovato sono effettivamente distinti: infatti, assumendo come operazione identica ogni cambiamento della base canonica che lasei inalterati $\mathrm{i}$ tre numeri $\alpha, \beta, \gamma$, è evidente che qualunque cambia- 
mento della detta base, che conservi soltanto la forma delle (1) e (2), equivale al prodotto di una operazione del tipo (3) per una operazione del tipo (4). 52. Caso di $p=3$.

Quando è $p=3$ le formole (1) e (2) dànno:

$$
\left(E_{5}^{u} E_{4}^{v}\right)^{3}=E_{5}^{3 u} E_{4}^{3 v} E_{1}^{u^{2} v}
$$

e quindi, eseguendo la trasformazione (4) ed osservando che è $u^{2} \equiv 1(\bmod 3)$, si vede subito che $\mathrm{i}$ numeri $\alpha$ e $\beta$ si portano rispettivamente in due numcri $\alpha_{1}$ e $\beta_{1}$ tali che:

$$
\alpha_{1} \equiv u \alpha+(\beta+1) v, \quad \beta_{1} \equiv \beta \quad(\bmod 3) .
$$

Dunque, se $\beta$ è 0 od 1 , si può determinare $v$ in modo che sia $\alpha_{1} \equiv 0(\bmod 3)$; ma se è $\beta=2$, disponendo di $u$ si può porrare $\alpha_{1}$ nel resto 1 oppure nel resto 0 di $p$.

Ottengo così quattro nuovi gruppi $G_{5}^{3,3}$ di grado 243 , i quali sono definiti dalle (1) e dalle formole (2) modificate come segue:

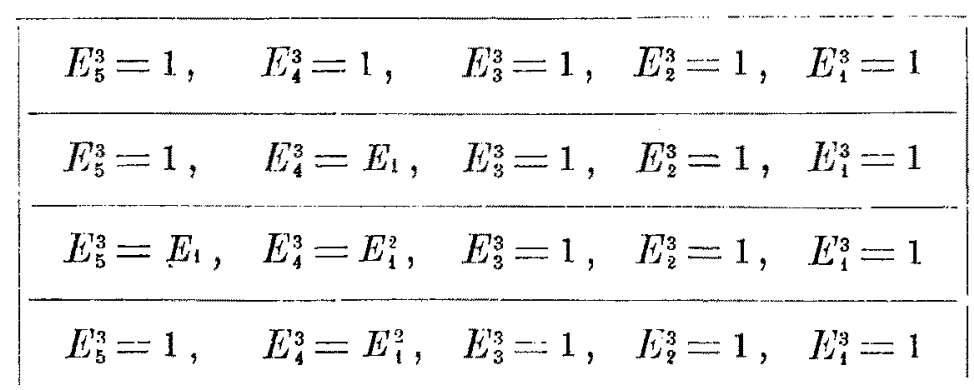

53. Caso di $p=2$.

Nel caso in cui è $p=2$ le relazioni (2) non si verificano.

Però, siccome l'elemento $E_{5}^{2}$ sta in $H$, la relazione:

$$
E_{0}^{-2} E_{4}^{\prime} E_{i}^{2}=E_{4} E_{2}^{2} E_{1}^{\prime}
$$

dà $E_{2}^{2}=E_{1}$. Allora la formola:

$$
E_{5}^{-1} E_{4}^{2} E_{50}^{2}=E_{4}^{2} E_{2}^{2}=E_{4}^{9} E_{1}^{\prime},
$$

mostra che l'elemento $E_{4}^{2}$ non è invertibile con $E_{s}^{\prime}$ e quindi il detto elemento appartiene a $K$ ma non ad $H$. L'elemento $H_{3}^{2}$ è invece permutabile con ogni elemento del gruppo principale e quindi sta in $H$.

Le (2) debbono perciò essere sostituite con le relazioni:

$$
E_{5}^{2}=E_{1}^{\alpha}, \quad E_{1}^{2}=E_{2} E_{1}^{\beta}, \quad E_{3}^{2}=E_{1}^{\prime}, \quad E_{2}^{2}=E_{1}^{\prime}, \quad E_{1}^{2}=1,
$$


le quali, insieme alle (1), definiscono effettivamente un gruppo $G_{5}^{3,3}$ di grado 32 qualunque siano gl'interi $\alpha, \beta, \%$

Ciò posto, io posso sempre supporre $y=0$, perchè, in caso contrario, raggiungo lo scopo eseguendo la trasformazione:

$$
\left(\begin{array}{ccccc}
E_{5}, & E_{4}, & E_{3}, & E_{2}, & E_{1} \\
E_{5} E_{4}, & E_{4}, & E_{3} E_{2}, & E_{2}, & E_{1}
\end{array}\right),
$$

la quale non altera le (1).

Poi, eseguendo la trasformazione:

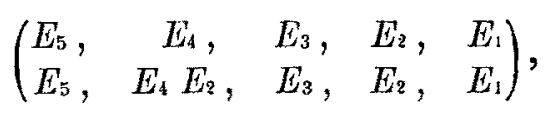

si vede che il numero $\beta$ si cambia in $\beta+1$ e quindi posso supporre $\beta=0$.

Si può d'altra parte verificare subito che tutti i cambiamenti della base canonica che lasciano inalterate le (1) e che conservano le ipotesi $\beta=\gamma=0$, non alterano $\alpha$.

Dunque, si hanno due gruppi distinti $G_{5}{ }^{33}$ di grado 32 , i quali sono definiti dalle (1) e dalle seguenti modificazioni delle formole (5):

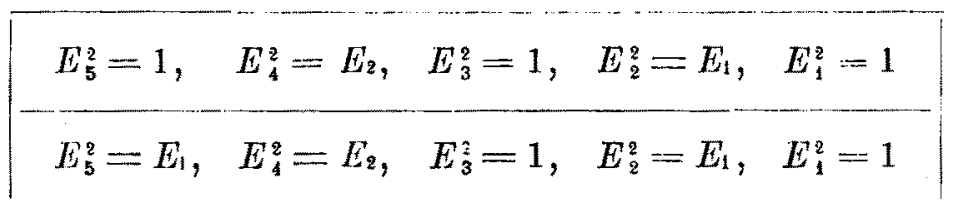

54. To debbo in ultimo luogo ricercare i gruppi $G_{5}^{3,4}$ e, in questa ricerca, mi fonderò sul fatto che, tutte le volte che è $p>2$, ogni gruppo $C_{i 5}^{3,4}$ contiene un divisore $G_{4}^{1}$ che ha tutti $\mathrm{i}$ suoi elementi di grado $p$.

Per stabilire ciò, si osservi anzitutto che, se $E, E^{*}$ sono due arbitrarî elementi di $G_{5}^{3,4}$ ed $E_{1}$ è un elemento generatore del gruppo $H=K$, si ha:

donde si ricava:

$$
E^{\prime-1} E^{\prime \prime} E^{\prime}=E^{\prime} E_{1}^{\prime \prime}
$$

$$
\left(E E^{\prime}\right)^{p}=E^{p} E_{1}^{\prime p} E_{1}^{h(p)}
$$

quindi, a vendo supposto $p>2$, risulta:

$$
\left(E E^{\prime}\right)^{p}=E^{p} E^{\prime} p \text {. }
$$

Sia ora $H_{2}$ un elemento di $C_{5}{ }^{34}$ fuori del gruppo $H$ ed $E_{3}$ un elemento fuori del gruppo generato dagli elementi $E_{2}, E_{1}$. Ponendo:

$$
E_{3}^{p}=E_{1}^{\prime \prime}, \quad E_{2}^{p}=E_{1}^{\beta},
$$


se l'intero $\beta$ è primo con $p$, l'elemento $E_{3}^{q} E_{\mathrm{a}}{ }^{a}$, che non sta in $H$, è di grado $p$ ed io lo assumo come elemento $E_{2}$.

Sia $E_{3}$ un elemento fuori del gruppo $G_{2}$ generato dagli élementi $E_{2}, E_{1}$ cos̀ definiti ed $E_{4}$ un elemento fuori del gruppo generato dagli elementi $E_{3}$, $E_{2}, E_{1}$. Ponendo:

$$
E_{4}^{p}=E_{1}^{\alpha}, \quad E_{3}^{p}=E_{1}^{\beta},
$$

se l'intero $\beta$ è primo con $p$, l'elemento $E_{4}^{\prime} E_{3}^{-\alpha}$, che non sta in $G_{2}$, è di grado $p$ ed io lo assumo come elemento $E_{3}$.

Sia $E_{4}$ un elemento fuori del gruppo $G_{3}$ generato dagli elementi $E_{3}, E_{2}, E_{1}$ così definiti, ed $E_{5}$ un elemento fuori del gruppo generato dagli elementi $E_{4}$, $E_{3}, E_{2}, E_{1}$. Ponendo:

$$
E_{5}^{p}=E_{1}^{\alpha}, \quad E_{4}^{p}=E_{1}^{\beta}
$$

se è $\beta$ primo con $p$, l'elemento $E_{5}^{f} E_{4}^{-a}$, che non sta in $G_{3}$, è di grado $p$ ed io lo assumo come elemento $E_{4}$.

Il gruppo $G_{4}$ generato dagli elementi $E_{4}, E_{3}, E_{2}, E_{1}$ cosi definiti ha tutti i suoi elementi di grado $p$ e possiede evidentemente tre divisori indipendenti d'indice $p$.

Il detto gruppo, per l'osservazione fatta al n. 40 non può essere Abeliano; quindi, giacchè non esistono (n. $\left.{ }^{\circ} 13\right)$ gruppi $G_{4}{ }^{2,3}$, il gruppo $G_{4}$ è un $G_{1}^{4}$.

Ciò posto, se $G_{2}$ è il divisore invertibile di $G_{4}^{1}$ ed $E_{5}^{\prime}$ è un elemento di $G_{5}^{3,4}$ fuori di $G_{4}^{1}$, si ha:

$$
\begin{gathered}
E_{5}^{-1} E_{4} E_{5}=E_{4} E_{1}^{2}, \quad E_{5}^{-1} E_{3} E_{5}=E_{3} E_{1}^{\prime \prime}, \quad E_{5}^{-1} E_{2} E_{5}=E_{2} E_{1}^{\prime h}, \\
E_{4}^{-1} E_{3} E_{4}=E_{3}^{\prime} E_{1}^{\prime}, \quad E_{4}^{-1} E_{2} E_{4}=E_{2}, \quad E_{4}^{-1} E_{2} E_{3}^{\prime}=E_{2}^{\prime}, \\
E_{5}^{\prime n}=E_{1}^{\prime \prime}, \quad E_{4}^{\prime \prime}=1, \quad E_{3}^{n}=1, \quad E_{2}^{p}=1, \quad E_{1}^{\prime}=1 .
\end{gathered}
$$

dove l'intero $h$ è necessariamente primo con $p$, altrimenti l'elemento $E_{2}$ risulterebbe permutabile con ogni elemento del gruppo principale $G_{5}^{3,4}$.

Chiamando $E_{2}$ una conveniente potenza di $E_{2}$, io posso ritenere $l=1$; poi, chiamando $E_{4}, E_{3}$ ordinatamente gli elemonti $E_{4}^{2} E_{3}^{-i}, E_{3} E_{2}^{-n}$, mi posso ridurre al caso di $\lambda=\mu=0$.

Si hanno quindi le formole:

$$
\begin{aligned}
& E_{5}^{-1} E_{4} E_{5}=E_{4}, \quad E_{5}^{-1} E_{3} E_{5}=E_{3}, \quad E_{5}^{-1} E_{2} E_{5}=E_{2} E_{1}, \\
& E_{4}^{-1} E_{3} E_{4}=E_{3} E_{1}, \quad E_{1}^{-1} E_{2} E_{4}^{\prime}=E_{2}, \quad E_{3}^{-1} E_{2} E_{3}=E_{2} .
\end{aligned}
$$

Se l'intero $\alpha$ è un multiplo di $p$, il gruppo principale ha tutti $j$ suoi elementi di grado $p$. Se invece a è primo con $p$, eseguendo la trasforma- 
zione:

$$
\left(\begin{array}{lllll}
E_{5}, & E_{4}, & E_{3}, & E_{2}, & E_{1} \\
E_{5}, & E_{4}, & E_{3}^{a}, & E_{2}^{a}, & E_{1}^{a}
\end{array}\right)
$$

porto a nel resto 1 di $p$.

Dunque, se è $p>2$, si hanno due soli gruppi $G_{5}^{3,4}$, i quali sono definiti dalle (6) e dalle formole:

$$
\begin{array}{llll}
E_{5}^{p}=1, & E_{4}^{p}=1, \quad E_{3}^{p}=1, & E_{2}^{p}=1, & E_{1}^{p}=1 \\
\hline E_{\mathrm{5}}^{p}=E_{1}, & E_{1}^{p}=1, \quad E_{3}^{p}=1, & E_{2}^{p}=1, & E_{1}^{p}=1 \\
\hline
\end{array}
$$

55. Caso $d i p=2$.

Quando ̀̀ $p=2$, chiamo $E_{2}$ un elemento di $G_{5}{ }^{3,4}$ fuori del gruppo $H=K$ e chiamo $E_{3}$ un elemento permutabile con $E_{2}$ fuori del gruppo generato dagli elementi $E_{2}, E_{1}$. Se $E_{2}$ ed $E_{3}$ sono entrambi di grado 4 , l'elemento $E_{3} E_{2}$ è di grado 2 ed io lo assumo come elemento $E_{2}$. Poi si consideri il gruppo $G_{1}^{t}$ costituito da tutti quegli elementi del gruppo principale che sono invertibili col detto elemento $E_{2}$.

Allora, se $E_{3}$ è un elemento di $G_{4}^{1}$ fuori del gruppo generato da $E_{2}, E_{1}$, ed $E_{4}$ è un elemento di $G_{4}^{1}$ fuori del gruppo generato dagli clementi $E_{3}$, $E_{2}^{\prime}, L_{1}$; finalmente, se $E_{\bar{z}}$ è un elemento del gruppo principale fuori di $G_{4}^{4}$, si hanno le formole:

$$
\begin{gathered}
E_{5}^{-1} E_{4} E_{5}=E_{4} E_{1}^{\lambda}, \quad E_{5}^{-1} E_{3} E_{5}=E_{3} E_{1}^{\mu}, \quad E_{5}^{-1} E_{2} E_{5}=E_{2} E_{1}, \\
E_{4}^{-1} E_{3} E_{4}=E_{3} E_{1}, \quad E_{4}^{-1} E_{2} E_{4}=E_{2}, \quad E_{3}^{-1} E_{2} E_{3}=E_{2}, \\
E_{5}^{9}=E_{1}^{\prime \prime}, \quad E_{4}^{2}=E_{1}^{\beta}, \quad E_{3}^{2}=E_{1}^{\gamma}, \quad E_{2}^{2}=1, \quad E_{1}^{2}=1,
\end{gathered}
$$

le quali definiscono sempre un gruppo $G_{5}^{3,4}$ di grado 32 .

Io posso supporre $\lambda=0$ altrimenti chiamo $E_{4}$ l'elemento $E_{4} E_{2}$ ed analogamente posso supporre $\mu=0$; inoltre, se occorre, chiamando $E_{5}$ l'elemento $E_{5} E_{2}$, posso ritenere $\alpha=0$.

Si ritrovano quindi le formole (6) e le formole:

$$
E_{5}^{2}=1, \quad E_{4}^{2}=E_{1}^{\beta}, \quad E_{3}^{2}=E_{1}^{\gamma}, \quad E_{2}^{2}=1, \quad E_{1}^{2}=1 \text {. }
$$

Se uno dei due numeri $\beta, y$ che vi figurano è zero, si può ritenere che sia $\gamma=0$, perchè, in caso contrario, si scambierebbe $E_{4}^{\prime}$ con $L_{2}^{\prime}$; il che non altera le (6). Allora chiamando $E_{4}$ l'elemento $E_{4} E_{3}$, si può ritenere anche $\beta=0$. 
Se invece $\beta$ e $\gamma$ hanno entrambi il valore 1 , è facile verificare che nessun cambiamento della base canonica che conservi la forma delle (6) e (7) altera $\beta$ e $\gamma$.

Dunque, anche quando è $p=2$, esistono due soli gruppi $G_{5}^{3,4}$ di grado 32 , i quali sono definiti dalle (6) e dalle seguenti modificazioni delle formole (7):

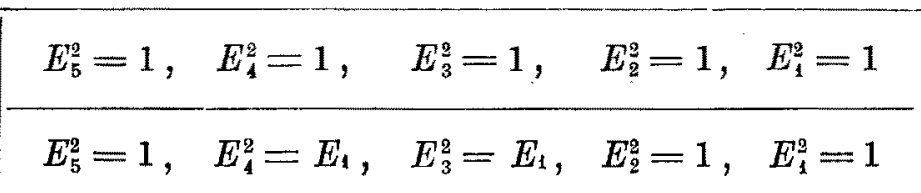

56. Riepilogo brevemente i risultati ottenuti nel presente paragrafo.

Per tutti i valori del numero primo $p>2$ esistono cinque, e non più, gruppi $G_{5}{ }^{3.3}$; invece, quando è $p=2$, esistono soltanto due gruppi $G_{5}{ }^{3,3}$ di grado 32.

Qualunque sia il numero primo $p$ esistono soltanto $d u e$ gruppi $G_{5}^{3,4}$.

Tutti $\mathrm{i}$ gruppi che si sono presentati in questo paragrafo sono rappresentati nella seguente tabella:

I.

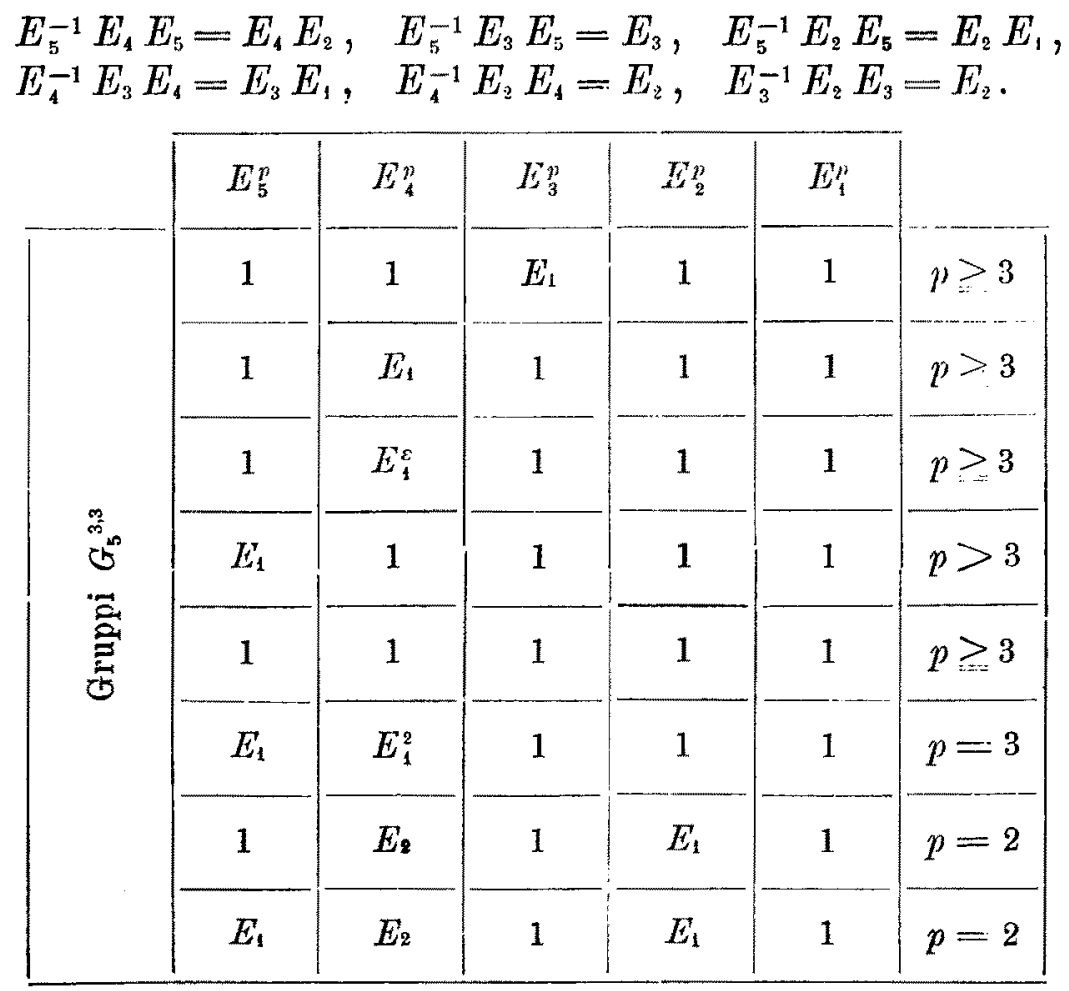


II.

$$
\begin{array}{ll}
E_{5}^{-1} E_{4} E_{5}=E_{4}, & E_{5}^{-1} E_{3} E_{5}=E_{3}, \quad E_{5}^{-1} E_{2} E_{5}=E_{2} E_{4}, \\
E_{4}^{-1} E_{3} E_{4}=E_{3} E_{1}, & E_{4}^{-1} E_{2} E_{4}=E_{2}, \quad E_{3}^{-1} E_{2} E_{3}=E_{2}
\end{array}
$$

\begin{tabular}{|c|c|c|c|c|c|c|}
\hline & $E_{5}^{p}$ & $E_{4}^{p}$ & $E_{3}^{p}$ & $E_{2}^{p}$ & $E_{1}^{p}$ & \\
\hline \multirow{3}{*}{ 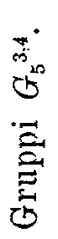 } & 1 & 1 & 1 & 1 & 1 & $p \geq 2$ \\
\hline & $F_{1}$ & 1 & 1 & 1 & 1 & $p>2$ \\
\hline & 1 & $E_{\mathrm{t}}$ & $E_{l}$ & 1 & 1 & $p=2$ \\
\hline
\end{tabular}

\section{§ VIII. Conclusione.}

57. Tutti i possibili gruppi di grado $2^{5}=32$ sono cinquanta. Di questi 50 gruppi, 22 tipi figurano nei quadri I e II alla fine del $\S$ III, altri 16 tipi nel quadro II alla fine del $\S \mathrm{V}$, altri 8 tipi nel quadro I alla fine del $\S \mathrm{VI}$ e finalmente altri 4 tipi figurano nei quadri I e II alla fine del precedente paragrafo.

Tutti i possibili gruppi di grado $3^{5}=243$ sono sessantasei. Di questi 66 gruppi, 22 tipi figurano nei quadri I e II alla fine del $\S$ III, altri 7 tipi nel quadro II alla fine del $§$ IV, altri 24 tipi nei quadri I e III alla fine del $\S \mathrm{V}$, altri 6 tipi nel quadro II alla fine del $\S$ VI e finalmente altri 7 tipi figurano nei quadri I e II alla fine del $\S$ VII.

Il numero di tutti i possibili gruppi di grado $p^{5}$, essendo $p>3$, ̀̀ uno dei quattro numeri:

$$
2 p+65,2 p+67,2 p+69,2 p+71,
$$

secondo che il numero primo $p$ soddisfa, ordinatamente, ad una delle quattro congruenze:

$$
p \equiv-1, \quad p \equiv 5, \quad p \equiv-5, \quad p \equiv 1 \quad(\bmod 12) .
$$

Nei quadri I e II alla fine del $\S$ III figurano 22 tipi di gruppi di grado $p^{5}$, altri $p+7$ tipi figurano nel quadro I alla fine del $\S$ IV, altri $p+21$ tipi nei quadri I e III alla fine del $\S \mathrm{V}$ ed altri 7 tipi nei quadri I e II alla 
fine del $\S$ VII. Finalmente, nel quadro III alla fine del $\S$ VI figurano 8, $10,12,14$ tipi di gruppi di grado $p^{5}$, secondo che il numero primo $p$ soddisfa, ordinatamente, alla $1^{\mathrm{a}}, 2^{\mathrm{a}}, 3^{\mathrm{a}}, 4^{\mathrm{a}}$ delle precedenti congruenze.

Nella tabella che segue sono scritti i numeri dei diversi tipi di gruppi di grado $p^{5}$ contenuti in ciascuna classe $G_{5}^{2, \mu}$ in corrispondenza alle varie forme del numero primo $p$.

\begin{tabular}{|c|c|c|c|c|c|c|}
\hline & $p=2$ & $p=3$ & $p=12 m-1$ & $p=12 m+5$ & $p=12 m-5$ & $p=12 m+1$ \\
\hline$G_{5}^{0,1}$ & 1 & 1 & 1 & 1 & 1 & 1 \\
\hline$G_{5}^{0,2}$ & 2 & 2 & 2 & 2 & 2 & 2 \\
\hline$G_{5}^{0,3}$ & 2 & 2 & 2 & 2 & 2 & 2 \\
\hline$G_{5}^{0,4}$ & 1 & 1 & 1 & 1 & 1 & 1 \\
\hline$G_{5}^{0,5}$ & 1 & 1 & 1 & 1 & 1 & 1 \\
\hline$G_{5}^{1,2}$ & 5 & 5 & 5 & 5 & 5 & 5 \\
\hline$G_{5}^{1,3}$ & 7 & 7 & 7 & 7 & 7 & 7 \\
\hline$G_{5}^{1,4}$ & 3 & 3 & 3 & 3 & 3 & 3 \\
\hline$G_{5}^{2,2}$ & 5 & 16 & $p+16$ & $p+16$ & $p+16$ & $p+16$ \\
\hline$G_{5}^{2,3}$ & 11 & 15 & $p+12$ & $p+12$ & $p+12$ & $p+12$ \\
\hline$G_{5}^{3,2}$ & 8 & 6 & 8 & 10 & 12 & 14 \\
\hline$G_{5}^{3,3}$ & 2 & 5 & 5 & 5 & 5 & 5 \\
\hline$G_{5}^{3,4}$ & 2 & 2 & 2 & 2 & 2 & 2 \\
\hline
\end{tabular}

Palermo, Marzo 1898. 